\title{
CHEMICAL SUBSTRUCTURE IN THE MILKY WAY HALO: A NEW POPULATION OF OLD STARS ${ }^{1}$
}

\author{
Inese I. Ivans, ${ }^{2,3,4,5}$ Christopher Sneden, ${ }^{2}$ C. Renée James, ${ }^{2,6}$ George W. Preston, ${ }^{7}$ Jon P. Fulbright, ${ }^{7}$ \\ Peter A. Höflich, ${ }^{2}$ Bruce W. Carney, ${ }^{3,8}$ and J. Craig Wheeler ${ }^{2}$ \\ Received 2003 February 3; accepted 2003 April 10
}

\begin{abstract}
We report the results of a coherent study of a new class of halo stars defined on the basis of the chemical compositions of three metal-poor objects $([\mathrm{Fe} / \mathrm{H}] \simeq-2)$ that exhibit unusually low abundances of $\alpha$-element $(\mathrm{Mg}, \mathrm{Si}, \mathrm{Ca})$ and neutron-capture ( $\mathrm{Sr}, \mathrm{Y}, \mathrm{Ba})$ material. Our analyses confirm and expand on earlier reports of atypical $\alpha$ - and neutron-capture abundances in BD $+80^{\circ} 245$, G4-36, and CS 22966-043. We also find that the latter two stars exhibit unusual relative abundance enhancements within the iron peak $(\mathrm{Cr}, \mathrm{Mn}, \mathrm{Ni}, \mathrm{Zn})$, along with what may be large abundances of $\mathrm{Ga}$, an element not previously reported as being observed in any metal-poor star. These results provide further evidence that chemical enrichment and star formation histories varied from region to region within the Milky Way halo. Comparing the chemical abundances of the newly identified stellar population to supernova model yields, we derive supernova ratios of Type Ia versus Type II events in the range of $0.6 \lesssim\left(N_{\text {Ia }} / N_{\text {II }}\right)_{\text {New Pop }} \lesssim 1.3$. For the Sun, we derive $0.18 \pm 0.01<\left(N_{\text {Ia }} / N_{\text {II }}\right)_{\odot}<$ $0.25 \pm 0.06$, supernova ratios in good agreement with values found in the literature. Given the relatively low metallicity and relatively high $\left\langle N_{\text {Ia }} / N_{\text {II }}\right\rangle$ ratios of the low- $\alpha$ stars studied here, these objects may have been born from material produced in the yields of the earliest Type Ia supernova events. We also report the results of a preliminary attempt to employ the observed chemical abundances of low-metallicity stars in the identification, and possible cosmic evolution, of Type Ia supernova progenitors, and we discuss the limitations of current model yields.
\end{abstract}

Subject headings: Galaxy: abundances — Galaxy: halo nuclear reactions, nucleosynthesis, abundances - stars: abundances stars: Population II — supernovae: general

On-line material: color figures, machine-readable table

\section{INTRODUCTION}

For high-precision cosmology, supernovae seem to be the most promising reliable standard candle candidates. One of the main uncertainties in their current use is the lack of knowledge regarding the evolutionary effects of the sample or of individual candles. Probing the properties of these

\footnotetext{
${ }^{1}$ Based on data acquired at the following facilities: McDonald Observatory, which is operated by the University of Texas at Austin; Las Campanas Observatory, which is operated by the Observatories of the Carnegie Institution of Washington; W. M. Keck Observatory, which is operated as a scientific partnership among the California Institute of Technology, the University of California, and NASA; and Kitt Peak National Observatory, National Optical Astronomy Observatory, which is operated by the Association of Universities for Research in Astronomy (AURA), Inc., under cooperative agreement with the National Science Foundation (NSF).

${ }^{2}$ Department of Astronomy and McDonald Observatory, University of Texas, Austin, TX 78712; chris@verdi.as.utexas.edu, pah@astro.as.utexas.edu,wheel@astro.as.utexas.edu.

${ }^{3}$ Visiting Astronomer, Kitt Peak National Observatory, National Optical Astronomy Observatory (NOAO), which is operated by the Association of Universities for Research in Astronomy (AURA), Inc., under cooperative agreement with the National Science Foundation (NSF).

${ }^{4}$ Hubble Fellow.

${ }^{5}$ Current address: Department of Astronomy, California Institute of Technology, MS 105-24, Pasadena, CA 91125; iii@astro.caltech.edu.

${ }^{6}$ Current address: Department of Physics, Sam Houston State University, Huntsville, TX 77341; phy_crj@shsu.edu.

${ }^{7}$ Carnegie Observatories, Carnegie Institution of Washington, 813 Santa Barbara Street, Pasadena, CA 91101; gwp@ociw.edu, jfulb@ociw.edu.

${ }^{8}$ Department of Physics and Astronomy, University of North Carolina, CB 3255 Phillips Hall, Chapel Hill, NC 27599; bruce@physics.unc.edu.
}

objects and their progenitors can be done by direct observations at high redshifts or, alternatively, by studying the signatures of the explosions and their influence on objects with long lifetimes that were formed at relatively early times. In low-mass stars, most of the elements are not produced in situ but, rather, are inherited from the stellar explosions of earlier generations of stars. The abundance determinations of low-mass stars thus provide information not otherwise available.

The main chemical contributors to the interstellar medium (ISM) at the earliest Galactic times were presumably the most massive and shortest lived stars. Their resulting Type II supernova (SN II) explosions (e.g., Tsujimoto et al. 1995; Woosley \& Weaver 1995) enriched the medium with light $\alpha$-elements (e.g., $\mathrm{O}, \mathrm{Ne}, \mathrm{Mg}$, and $\mathrm{Si}$ ), odd- $Z$ (e.g., $\mathrm{Na}, \mathrm{Al}$, and $\mathrm{P}$ ) and lesser amounts of other elements, including the heavier $\alpha$-elements (e.g., $\mathrm{Ca}, \mathrm{Ti}$ ), among others. Associated neutron-rich sites produced additional elements via rapid neutron-capture nucleosynthesis (" $r$-process"; e.g., Eu) by building heavier isotopes more quickly than $\beta$ decays occurred. Possible sites for the $r$-process include supernova winds (e.g., Thompson, Burrows, \& Meyer 2001; Terasawa et al. 2002; Wanajo et al. 2002 and references therein), disks and jets (e.g., Cameron 2001 and references therein), and/or neutron star mergers (e.g., Freiburghaus, Rosswog, \& Thielemann 1999; Rosswog et al. 1999 and references therein). Evolving more slowly, lower mass stars began to contribute their ejecta at later Galactic times, changing the overall composition of the interstellar mix, adding more Fe peak nuclei via Type Ia supernova (SN Ia) explosions, as well as those isotopes created in relatively slow neutron-capture processes (" $s$-process," where 
production of heavier elements takes place with a neutron flux rate low enough for $\beta$-decays to take place). Thus, yields from the lower mass SNe Ia and asymptotic giant branch (AGB) stars were incorporated into material that had previously been enriched by SNe II and their associated neutron-rich sites.

The general view of Galactic chemical evolution is that $\mathrm{SNe}$ Ia contributed at an epoch later than the initial chemical enrichment by SNe II. This is shown by the delay in the production of iron (Tinsley 1979). However, the stage in Galactic evolution at which SNe Ia began to contribute is not known precisely. Major SN Ia contributions likely arose subsequent to those from AGB star processing. A rise in the abundance of elements whose principal isotopes are created in the $s$-process (e.g., $\mathrm{Sr}$ and $\mathrm{Ba}$; " $s$-process elements") is observed in the range of $-3 \lesssim[\mathrm{Fe} / \mathrm{H}] \lesssim-2$ (see, e.g., Spite $\&$ Spite 1978, their Fig. 5; Burris et al. 2000, their Figs. 5, 7, and 9). However, the overall Galactic trend of a lowering of the $[\alpha / \mathrm{Fe}]^{9}$ ratio is not observed until $[\mathrm{Fe} / \mathrm{H}] \simeq-1$ (see e.g., Pagel \& Tautvaisiene 1995, their Fig. 3; McWilliam 1997, his Fig. 1).

A number of studies have uncovered stars of intermediately low metallicities that possess relatively low $[\alpha / \mathrm{Fe}]$, $[\mathrm{Na} / \mathrm{Fe}]$, and/or neutron-capture element ratios when compared to other stars of comparable metallicities, with abundance ratio differences in the range of $0.1-0.6$ dex. The literature includes the following: a sample of kinematically selected high-velocity disk and halo field stars $(-1.3<[\mathrm{Fe} / \mathrm{H}]<-0.4 ;$ Nissen \& Schuster 1997), an outer halo star (as defined by its large apogalacticon distance, “ $R_{\text {apo }}$ ") BD $-06^{\circ} 855([\mathrm{Fe} / \mathrm{H}] \sim-0.7$; Carney \& Latham $1985)$, the outer halo common proper-motion pair HD 134439 and HD $134440([\mathrm{Fe} / \mathrm{H}] \simeq-1.5$; King 1997), and the relatively young outer halo globular clusters $\mathrm{Pal} 12$ and Rup $106([\mathrm{Fe} / \mathrm{H}] \sim-1$ and -1.4 , respectively; Brown, Wallerstein, \& Zucker 1997). In all of these analyses, the abundances and kinematics of the objects have been found to be consistent with the idea of accretion events in the outer halo.

In studies encompassing larger samples of halo stars, lower $[\alpha / \mathrm{Fe}]$ ratios on average are found for stars with high space velocities with respect to the local standard of rest (Fulbright 2002) and for outer halo stars (Stephens \& Boesgaard 2002). It has been suggested that the kinematically extreme stars likely formed at large distances from the Galactic center in localized star-forming regions or protogalactic fragments that were later assimilated or accreted into the halo. However, Fulbright (2002) and Stephens \& Boesgaard (2002) find that the abundance ratio patterns of these stars are not consistent with those of stars in the present-day dwarf spheroidal (dSph) galaxies. Both studies note that the kinematically extreme stars in their samples are not likely to be the result of mergers with populations such as those currently orbiting the Galaxy.

Recently, there have been a few serendipitous discoveries of lower metallicity Galactic halo stars $([\mathrm{Fe} / \mathrm{H}] \simeq-2)$ that show severe departures (over 1 dex in some cases) from the general enhanced $\alpha$-element chemical abundance trends of

\footnotetext{
${ }^{9} \mathrm{We}$ adopt the usual spectroscopic notations that $[\mathrm{A} / \mathrm{B}] \equiv$ $\log _{10}\left(N_{\mathrm{A}} / N_{\mathrm{B}}\right)_{*}-\log _{10}\left(N_{\mathrm{A}} / N_{\mathrm{B}}\right)$ and $\log \epsilon(\mathrm{A}) \equiv \log _{10}\left(N_{\mathrm{A}} / N_{\mathrm{H}}\right)+12.0$, for elements $\mathrm{A}$ and $\mathrm{B}$. In addition, metallicity is defined as the stellar $[\mathrm{Fe} / \mathrm{H}]$ value.
}

the halo: BD $+80^{\circ} 245$ (Carney et al. 1997), G4-36 (James 1998, 2000), and CS 22966-043 (Preston \& Sneden 2000). These objects possess similar abundances: they display underabundances in the $[\alpha / \mathrm{Fe}]$ ratios with respect to the scaled solar values and do not fit the relation shown by other stars of similar metallicities for neutron-capture elements. In this study, we have analyzed these stars as a group, with the twofold aim of uncovering the nucleosynthetic history of this new population of old stars and probing the properties of SN Ia progenitors in a variety of scenarios.

The organization of this paper is as follows. In $\S 2$ we summarize the characteristics of the program stars that prompted our interest, give details of our new spectroscopic observations, and present the equivalent width (EW) measurements that went into the analysis. In $\S 3$ we outline our analysis techniques and abundance results and compare the results against both those found in other studies of the same stars and those of the halo field star population. In $\S 4$ we report the results of our investigations to explain the derived abundances, including those we obtained by modeling the abundances in the context of various supernova models, and discuss the possibility of multiple generations of anomalous stars for which similar but milder abundance trends have been uncovered in the literature. Finally, in $\S 5$ we summarize our current understanding of the nucleosynthetic history of our program stars.

\section{OBSERVATIONAL MATERIAL}

Our high-resolution spectroscopic data were acquired at a number of facilities. Observational data for our stars are presented in Table 1. In this section we describe the spectroscopic observations, reductions, and measurements employed in this study.

\section{1. $B D+80^{\circ} 245$}

In their "discovery of an 'alpha'-element-poor halo star," Carney et al. (1997) found that $\mathrm{BD}+80^{\circ} 245$ possessed a low $[\mathrm{O} / \mathrm{Fe}]$ ratio $(0.4$ dex below the average found in other metal-poor stars), a very low $[\alpha / \mathrm{Fe}]$ ratio $(0.7 \mathrm{dex}$ below average), and an extremely deficient $[\mathrm{Ba} / \mathrm{Fe}]$ ratio (1.8 dex below average). A follow-up analysis of this star was done by Fulbright $(1999,2000,2002)$ using Keck data. In addition to confirming the low elemental abundances discovered by Carney et al. (1997), Fulbright found an extremely deficient $[\mathrm{Eu} / \mathrm{Fe}]$ ratio $(\sim 0.8$ dex below average).

We acquired higher resolution data $(R \equiv \lambda / \Delta \lambda \simeq$ $60,000)$ of this object at McDonald Observatory. The McDonald data were gathered with the " $2 \mathrm{~d}$-coudé" echelle (Tull et al. 1995) at the Harlan J. Smith telescope. The spectrograph was set in the cs23-e2 configuration using a Tektronix $2048 \times 2048$ CCD chip with $24 \mu \mathrm{m}$ pixels and a 1 " slit width. The total wavelength coverage is $\sim 3800-8700 \AA$, with a restricted useful spectral range due to chip sensitivity issues and order interstice losses increasing redward from $\sim 5800 \AA$ A. Our integrations achieved a signal-to-noise ratio $(\mathrm{S} / \mathrm{N})_{\text {。 }}$ of $\sim 100$ pixel $^{-1}$ at $6645 \AA$, decreasing to $\sim 35$ at $4130 \AA$.

A standard set of calibration images, including those of tungsten filament lamp and thorium-argon hollow cathode lamps, biases, and darks, were taken on the same night as the program star data. We also obtained spectra of hot, rapidly rotating, essentially featureless stars ( $\delta$ Ori and $\zeta \mathrm{Oph}$ ) 


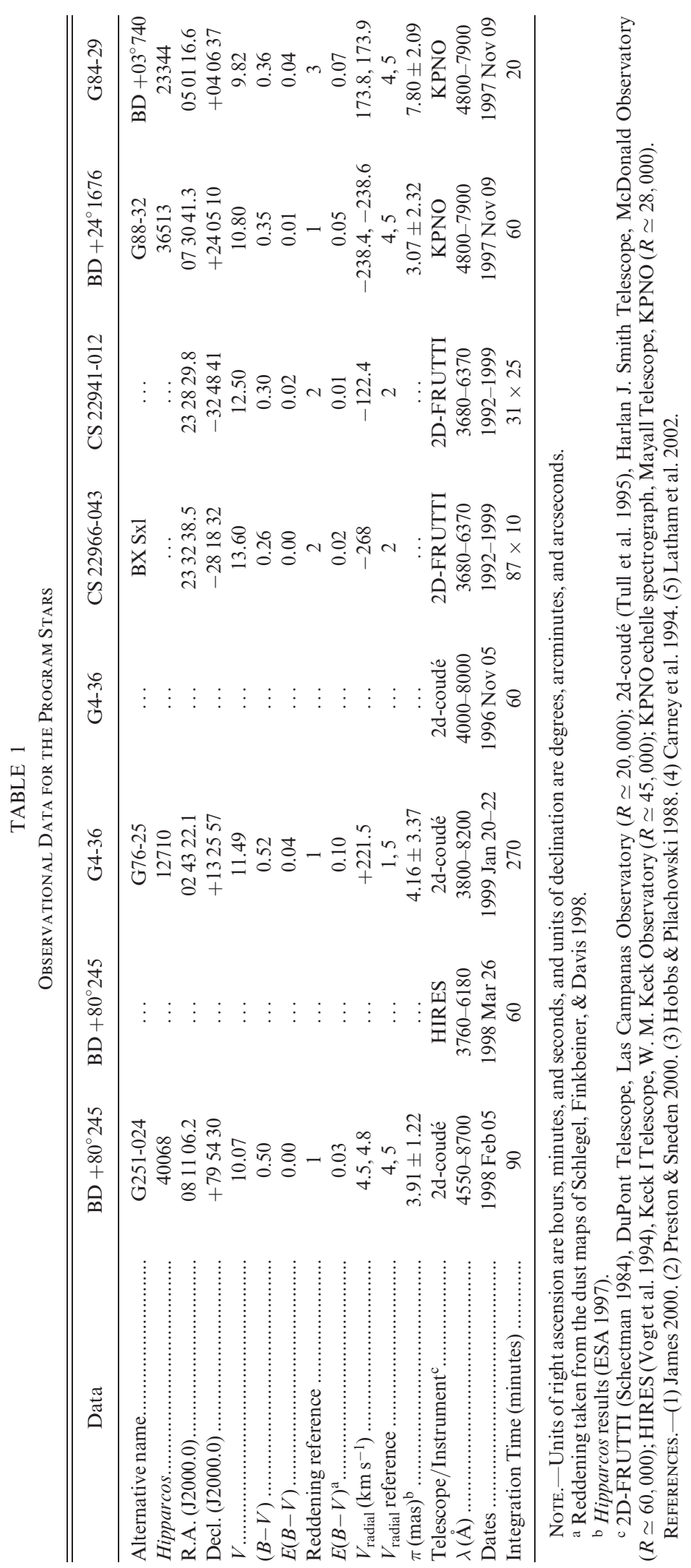


in order to aid in the removal of telluric features from the program star data. Reductions performed using IRAF ${ }^{10}$ tasks include the following: CCD bias structure subtraction, trimming of the CCD overscan region, bad pixel interpolation, division by a flat field to correct for pixel-to-pixel variations, subtraction of a low-order surface fit to the interorder light to remove the scattered light contribution, excision of most cosmic rays, extraction of a singledimensioned multiordered spectrum, and wavelength calibration using the thorium-argon integrations. We further employed a specialized software package, SPECTRE (Fitzpatrick \& Sneden 1987), to complete the reductions, which included continuum normalization, further cosmicray excision, and telluric feature division.

The Keck data for BD $+80^{\circ} 245$ utilized in the present study were acquired by Fulbright (1999). Data reduction up to and including wavelength calibration is described in Fulbright \& Kraft (1999). SPECTRE was subsequently employed as described above.

\section{2. $G 4-36$}

The unusual nature of this star was discovered by James $(1998,2000)$ in a study of the chemical abundances of high proper motion stars observed at McDonald Observatory. In addition to low $\alpha$-element and $\mathrm{Ba}$ abundances resembling those found in $\mathrm{BD}+80^{\circ} 245$, James reported unusually high $\mathrm{Ti}$ and $\mathrm{Zn}$ abundances. The discovery was made employing data acquired at $R \simeq 30,000$. In Figures 1 and 2, we display spectra of G4-36 against G25-24, stars possessing comparable stellar parameters. The data are taken from James (2000), who observed both stars with the same instrumental setup. To supplement those data, we gathered new spectra

${ }^{10}$ IRAF is distributed by NOAO, which is operated by AURA, Inc., under cooperative agreement with the NSF.

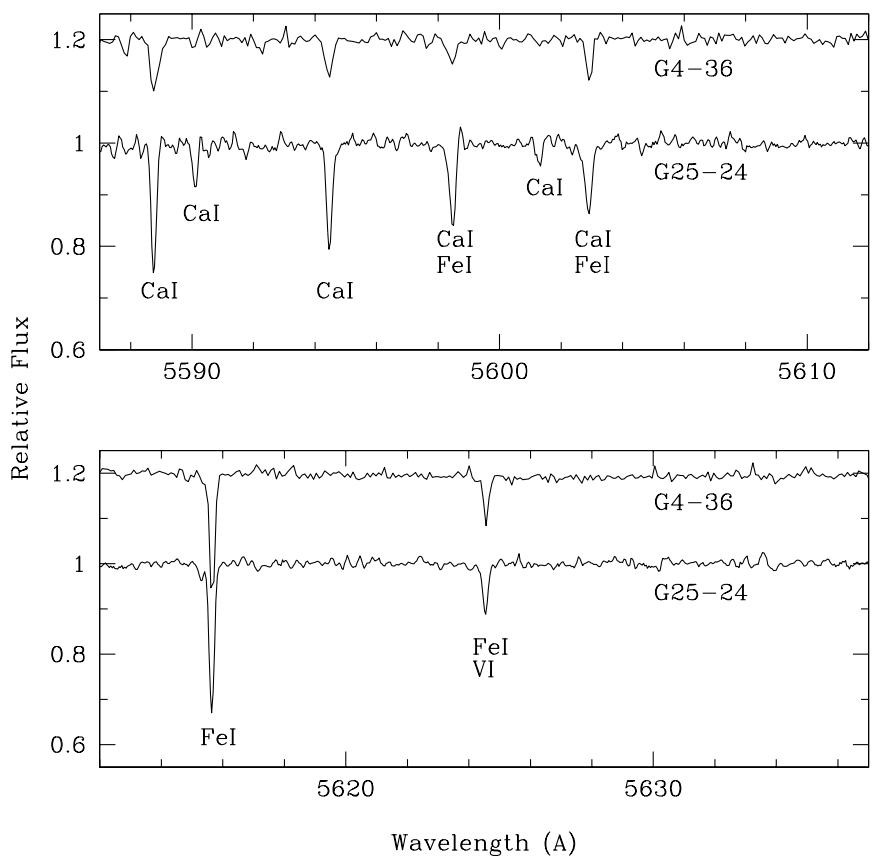

FIG. 1.- Spectral comparisons of $\mathrm{Ca}$ and $\mathrm{Fe}$ features in G4-36 and G2524, stars possessing comparable stellar parameters. A $50 \AA$ A region is split in two panels and illustrates the dramatic differences in the $\mathrm{Ca}$ abundances.

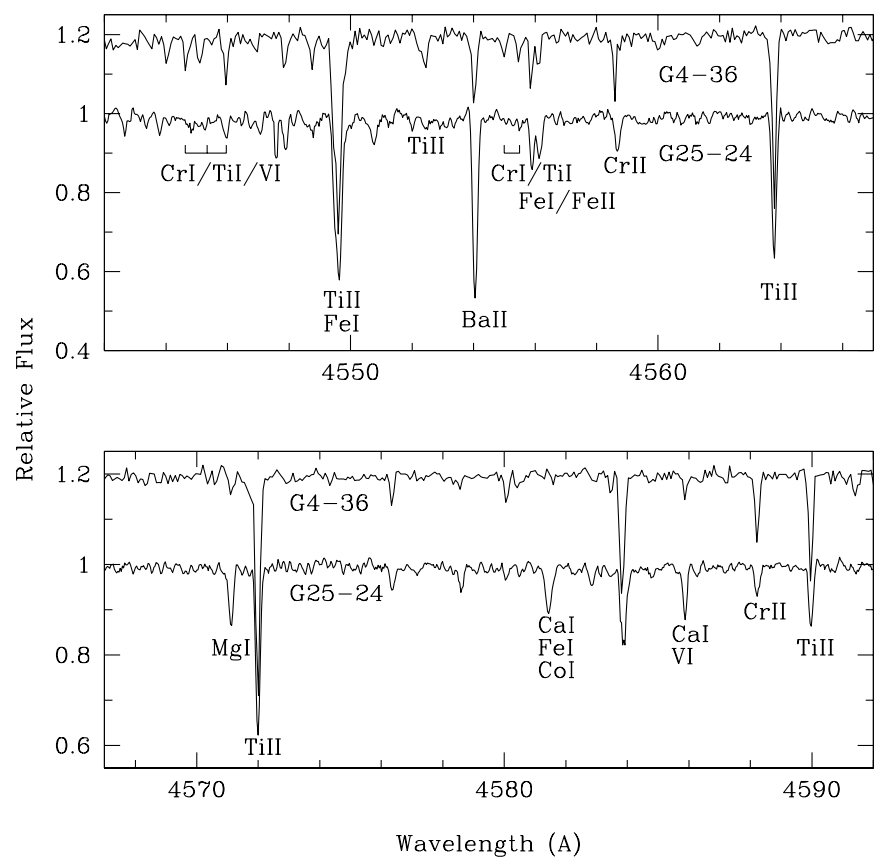

FIG. 2.-Spectral comparisons of $\alpha$-, Fe peak, and $s$-process elements in G4-36 and G25-24. A $50 \AA$ region is split in two panels. Overenhancements in G4-36 of Ti and $\mathrm{Cr}$ are easily detected. Also prominent are the differences in the abundances of $\mathrm{Mg}, \mathrm{Ca}$, and $\mathrm{Ba}$, which are all deficient in G4-36.

for G4-36 at a resolution of $R \simeq 60,000$. The new data have an $\mathrm{S} / \mathrm{N}$ of $\sim 170$ near $6645 \AA$ and 55 near $4130 \AA$. The spectrograph setup was the same as that of the McDonald data taken for $\mathrm{BD}+80^{\circ} 245$, as was the data reduction procedure.

\subsection{CS 22966-043}

The unusual chemical nature of CS 22966-043 was discovered by Preston \& Sneden (2000) in an analysis of data taken during a long-term observing campaign (1992-1999) of blue metal-poor (BMP) stars, during which over 1200 echelle spectra from Las Campanas Observatory were gathered using the 2D-FRUTTI detector (Schectman 1984) on the echelle spectrograph of the du Pont telescope. In the Preston \& Sneden (2000) study, this SX Phe variable star (Preston \& Landolt 1998) was found to have unusual $\alpha$ element deficiencies, combined with high $[(\mathrm{Cr}, \mathrm{Mn}, \mathrm{Ti}) / \mathrm{Fe}]$ abundance ratios and extremely low $[(\mathrm{Sr}, \mathrm{Ba}) / \mathrm{Fe}]$ ratios. The present study employs those data, which have been augmented by the co-addition of overlapping orders.

As a comparison object to our analysis of CS 22966-043, we employed another BMP star, CS 22941-012. This star possesses similar stellar parameters and, just as importantly, was observed using the same instrumental setup as the chemically unusual star. Thus, regardless of any systematics in the determination of our absolute abundances of the hot BMP stars, we are confident in their relative abundances. In Figures 3 and 4 we display spectra of both BMP stars.

Because of the sizable range in pulsation velocity and short pulsation period of CS 22966-043 ( 30 km s$)^{-1}$ and 54 minutes, respectively; Preston \& Landolt 1999), we combined the approximately 30 integrations of 10 minutes each obtained in the half-cycles centered on maximum and minimum light after Doppler-shifting each spectrum to the time-averaged velocity of the star. A preliminary analysis 


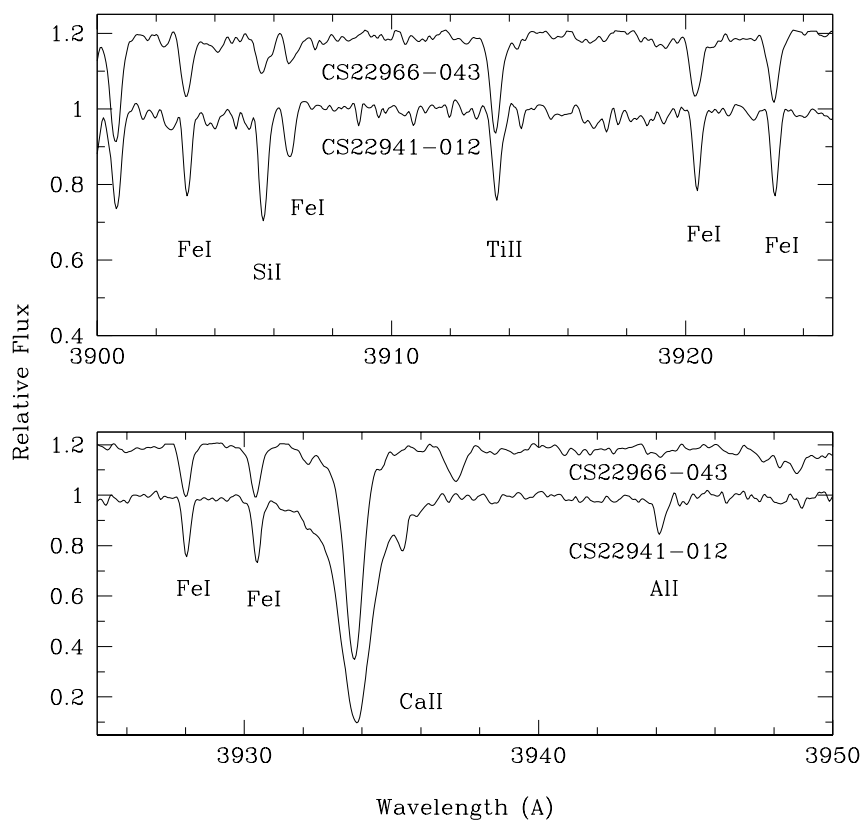

FIG. 3.- Spectral comparisons of light odd- $Z$ and $\alpha$-element features in CS 22966-043 and CS 22941-012, the two BMP stars of this study that possess similar stellar parameters. A $50 \AA$ region is split in two panels. The top panel illustrates the low Si I abundance for CS 22966-043; the bottom panel illustrates the differences in $\mathrm{Ca}$ II and $\mathrm{Al} \mathrm{I}$, both found to be deficient in CS 22966-043.

indicated no significant difference in abundances derived from these two co-added spectra, so they too were combined to further increase $\mathrm{S} / \mathrm{N}$. Additional details regarding the acquisition and reduction of the data for both of the BMP stars are reported in Preston \& Sneden (2000).

\subsection{G84-29 and $B D+24^{\circ} 1676$}

G84-29 $([\mathrm{Fe} / \mathrm{H}] \sim-3.0)$ and $\mathrm{BD}+24^{\circ} 1676([\mathrm{Fe} / \mathrm{H}] \sim$ $-2.7)$ are two stars for which some unusual abundances resembling those of G4-36 and CS 22966-043 have been reported in previous studies. King (1997) summarizes some of the previous literature results for G84-29.

We acquired spectra for these two stars with the echelle spectrograph of the Mayall Telescope at Kitt Peak National Observatory. The observations were taken with an instrumental configuration to include the oxygen triplet, as well as other $\alpha$-elements observable at red wavelengths. Using the red long camera and red collimator, a $2048 \times 2048$ chip with $24 \mu \mathrm{m}$ pixels, echelle grating 58.5-63, and the G226-1 crossdisperser, our wavelength coverage at $R \simeq 28,000$ is approximately 4800-7900 A. For both stars, our S/N near $6645 \AA$ is $\sim 150$ per resolution element, declining to $\sim 100$ near $5000 \AA$ for $\mathrm{G} 84-29$ and $\sim 90$ for $\mathrm{BD}+24^{\circ} 1676$. The data reduction procedure for these stars was the same as that employed for the data acquired at McDonald Observatory.

\subsection{Equivalent Width Measurements}

Using SPECTRE to measure the EWs, we adopted either the EW given by direct integration of the flux across a given line profile or the EW of a Gaussian profile fit to a given feature. The input line list (see Table 2) primarily relies on laboratory $\log g f$ values used in previous studies by our

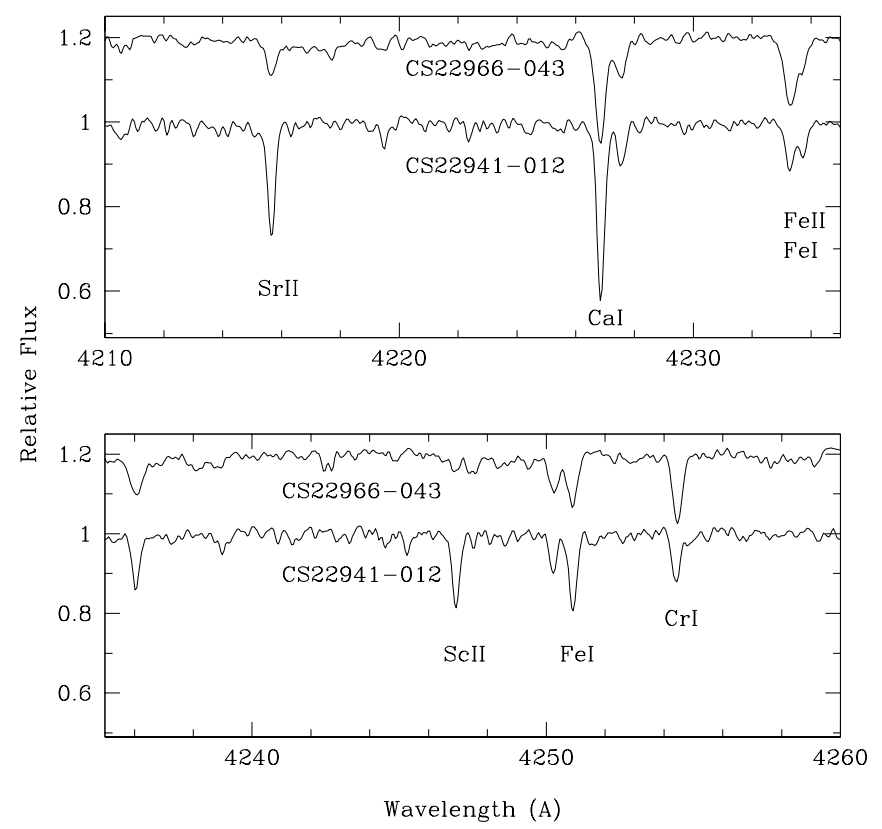

Fig. 4.- Spectral comparisons of Fe peak and $s$-process element features in CS 22966-043 and CS 22941-012. A 50 A region is split in two panels. The top panel displays a large difference in $\mathrm{Sr}$ II, as well as a less dramatic difference in Ca II, both of which are deficient in CS 22966-043. The bottom panel illustrates the deficiency of Sc II in CS 22966-043, as well as the slight overabundance of $\mathrm{Cr}$ I.

group, supplemented by additional literature sources. Of particular importance were the ionized iron lines taken from the study of the reliability of $\mathrm{Fe}$ II atomic parameters done by Lambert et al. (1996).

For each of the program stars, there exist EWs in the literature against which we may compare ours. We compared our EWs to those in common with other studies and find them to be in excellent agreement. A comparison of our EW measurements for the five main program stars and those of other studies is presented in Table 3. Column (3) shows the mean difference in the sense of $\Delta \mathrm{EW}=$ this study - other study, column (4) shows the standard deviation of the mean, column (5) shows the variance of a single measurement, and column (6) shows the number of lines in common between the two studies.

\section{CHEMICAL ABUNDANCE ANALYSIS}

All of the abundances were derived using the current version of the local thermodynamic equilibrium (LTE) line analysis code MOOG (Sneden 1973). As input to MOOG, we employed trial model atmospheres without overshooting from the Kurucz grid of models based on the 1995 version of the ATLAS9 code (Kurucz 1993 ${ }^{11}$ ) and the treatment described by Castelli, Gratton, \& Kurucz (1997), using a modified version of a Kurucz model interpolation program kindly supplied by A. McWilliam (2002, private communication). As shown by Peterson, Dorman, \& Rood (2001), the Kurucz models without overshooting better represent the observed ultraviolet spectrum in both the Sun and their sample of metal-poor dwarfs than do the models with overshooting.

\footnotetext{
11 The grids are available at http://kurucz.harvard.edu/grids.html.
} 
TABLE 2

EW Measurements

\begin{tabular}{|c|c|c|c|c|c|c|c|c|}
\hline $\begin{array}{c}\lambda \\
(\stackrel{\AA}{\mathrm{A}})\end{array}$ & $\begin{array}{c}\chi \\
(\mathrm{eV})\end{array}$ & $\log g f$ & $\mathrm{BD}+80^{\circ} 245$ & G4-36 & CS 22966-043 & CS 22941-012 & $\mathrm{BD}+24^{\circ} 1676$ & G84-29 \\
\hline \multicolumn{9}{|c|}{ Li I } \\
\hline $6707.76 \ldots \ldots \ldots \ldots$ & 0.000 & 0.000 & 17.7 & 32.7 & $\ldots$ & $\ldots$ & 26.4 & $\ldots$ \\
\hline \multicolumn{9}{|c|}{$\mathrm{O}_{\mathrm{I}}$} \\
\hline 7774.18 ................ & 9.140 & 0.148 & $\ldots$ & 7.5 & $\ldots$ & $\ldots$ & 19.5 & $\ldots$ \\
\hline 7771.95 ................ & 9.140 & 0.284 & $\ldots$ & 12.2 & $\ldots$ & $\ldots$ & 17.2 & $\ldots$ \\
\hline $7775.40 \ldots \ldots \ldots \ldots$ & 9.140 & -0.144 & $\cdots$ & 4.1 & $\cdots$ & $\cdots$ & 14.4 & $\ldots$ \\
\hline \multicolumn{9}{|c|}{$\mathrm{Na} \mathrm{I}$} \\
\hline $5895.94 \ldots \ldots \ldots \ldots . .$. & 0.000 & -0.191 & $\ldots$ & 112.1 & 40.0 & 72.9 & 60.7 & 42.3 \\
\hline $5889.97 \ldots \ldots \ldots \ldots$ & 0.000 & 0.112 & $\ldots$ & 131.2 & $\ldots$ & 97.4 & 79.8 & 60.0 \\
\hline
\end{tabular}

Note.-Table 2 is published in its entirety in the electronic edition of the Astrophysical Journal. A portion is shown here for guidance regarding its form and content.

With the exception of iron, our analysis relies on the solar (where available) or meteoritic abundances from the compilation by Anders \& Grevesse (1989). As in previous studies by our group, we adopt $\log \epsilon(\mathrm{Fe})=7.52$ in place of the Anders \& Grevesse (1989) iron abundance. This value is close to that recommended by Grevesse \& Sauval (1998) $[\log \epsilon(\mathrm{Fe})=7.50]$, and we refer the reader to discussions in Sneden et al. (1991b), Ryan, Norris, \& Beers (1996), and McWilliam (1997) where some of the alternative choices are summarized.

Except where otherwise noted, we adopted standard spectroscopic constraints in deriving the "best-fit" stellar parameters. To constrain the value of the effective temperature $\left(T_{\text {eff }}\right)$, we demanded that the derived abundance be the same for all lines of a given ion (e.g., neutral iron), regardless of their atomic parameters; i.e., we minimized the slope of the abundance as a function of the excitation potential. To constrain the value of the microturbulent velocity $\left(\xi_{t}\right)$, we forced all lines, regardless of their strength, to yield the same abundance; i.e., we minimized the slope of abundance as a function of the EWs. Furthermore, to constrain the value of the surface gravity $(\log g)$, we imposed the condi-

TABLE 3

EW Measurement Comparison

\begin{tabular}{|c|c|c|c|c|c|}
\hline $\begin{array}{c}\text { Star } \\
(1)\end{array}$ & $\begin{array}{c}\text { Source } \\
\text { (2) }\end{array}$ & $\begin{array}{c}\Delta \\
(\mathrm{m} \AA \\
(3)\end{array}$ & $\begin{array}{c}\sigma / \sqrt{N} \\
(\mathrm{~m} \AA) \\
(4)\end{array}$ & $\begin{array}{c}\sigma \\
(\mathrm{m} \AA) \\
(5)\end{array}$ & $\begin{array}{l}N \\
(6)\end{array}$ \\
\hline \multirow[t]{4}{*}{$\mathrm{BD}+80^{\circ} 245 \ldots \ldots \ldots \ldots$} & 1 & +1.5 & 0.9 & 3.4 & 14 \\
\hline & 2 & +1.5 & 0.4 & 3.4 & 60 \\
\hline & 3 & +1.3 & 0.8 & 5.6 & 51 \\
\hline & 4 & -0.3 & 0.6 & 5.2 & 89 \\
\hline G4-36.................... & 3 & -0.5 & 0.7 & 5.6 & 59 \\
\hline CS 22966-043 ........... & 5 & +3.0 & 1.2 & 8.1 & 42 \\
\hline CS 22941-012 ........... & 5 & +2.0 & 1.1 & 7.5 & 47 \\
\hline \multirow[t]{3}{*}{ G84-29 ..................... } & 6,7 & +0.3 & 0.7 & 3.2 & 24 \\
\hline & 2 & -0.9 & 0.6 & 2.0 & 13 \\
\hline & 8 & +2.2 & 1.1 & 4.4 & 15 \\
\hline $\mathrm{BD}+24^{\circ} 1676 \ldots \ldots \ldots$ & 4 & +0.5 & 0.7 & 4.1 & 34 \\
\hline
\end{tabular}

REFERENCES.- (1) Carney et al. 1997. (2) Fulbright 2000. (3) James 2000. (4) Stephens \& Boesgaard 2002. (5) Preston \& Sneden 2000. (6) Magain 1989. (7) Zhao \& Magain 1990. (8) Carretta et al. 2002. tion that all ionization states of a given element (e.g., iron) yield the same abundance. The stellar parameters we derived for the program stars in this study are presented in Table 4.

Figure 5 shows the derived abundances for the three low- $\alpha$ stars grouped by atomic number, in the context of the mean abundances found for stars of comparable metallicities. All three of our stars possess anomalously low light $\alpha$-element abundances, along with low abundances of $\mathrm{Sr}$ and $\mathrm{Ba}$. However, the low- $\alpha$ stars fall into two groups: they do not share

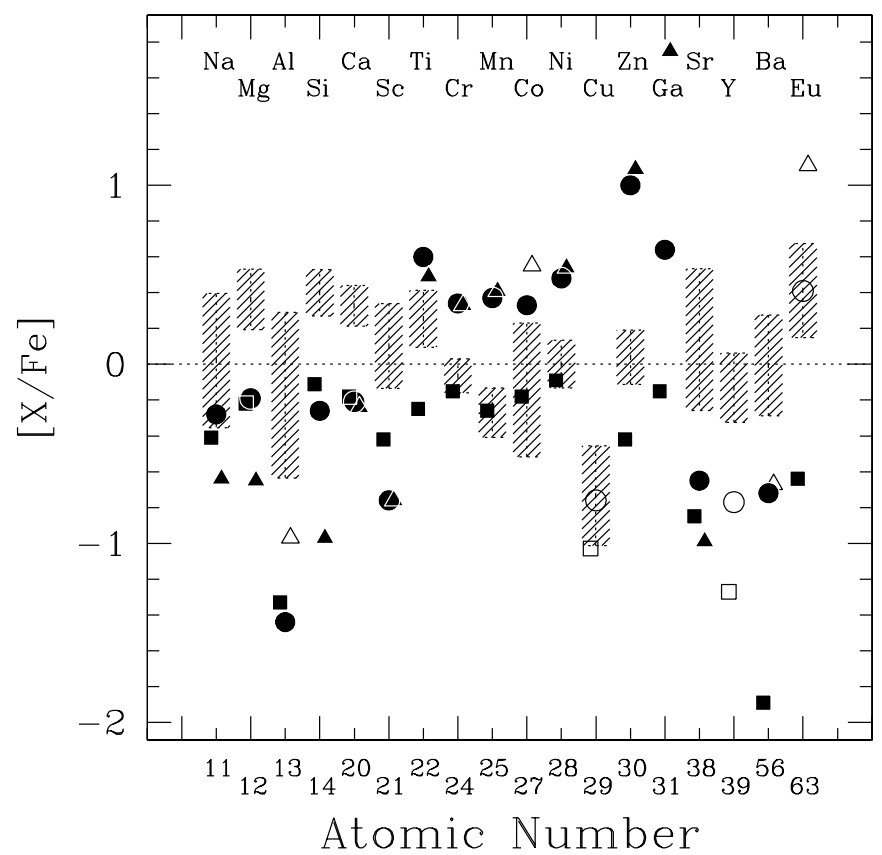

FIG. 5.-Abundances from Table 5 grouped by atomic number for BD $+80^{\circ} 245$ ( filled squares), G4-36 (filled circles), and CS 22966-043 (filled triangles). In order to display the abundances of the program stars more clearly, we have imposed small shifts in the abscissa. Mean abundances of halo stars of comparable metallicities $(-1.75<[\mathrm{Fe} / \mathrm{H}]<-2.25)$ are denoted by shaded regions that are centered on the average value and extend to $1 \sigma$ from the mean of a particular element. Strict upper limits on derived abundances of our program stars for specific elements are denoted by hollow symbols. [See the electronic edition of the Journal for a color version of this figure.] 
TABLE 4

Stellar Parameter Comparison

\begin{tabular}{|c|c|c|c|c|}
\hline Reference & $T_{\mathrm{eff}}$ & $\log g$ & $\xi_{t}$ & {$[\mathrm{Fe} / \mathrm{H}]$} \\
\hline \multicolumn{5}{|c|}{$\mathrm{BD}+80^{\circ} 245$} \\
\hline 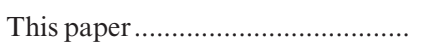 & 5225 & 3.00 & 1.25 & -2.07 \\
\hline Stephens \& Boesgaard 2002 .......... & 5569 & 3.47 & 1.56 & -1.76 \\
\hline 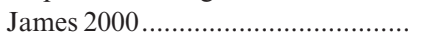 & 5350 & 3.20 & 1.50 & -2.00 \\
\hline 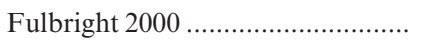 & 5225 & 3.00 & 1.35 & -2.05 \\
\hline Carney et al. 1997 ............................. & 5400 & 3.20 & 1.50 & -1.86 \\
\hline \multicolumn{5}{|c|}{ G4-36 } \\
\hline This paper.... & 5975 & 4.05 & 1.15 & -1.94 \\
\hline 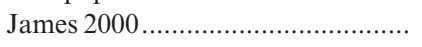 & 5900 & 4.20 & 1.70 & -2.00 \\
\hline 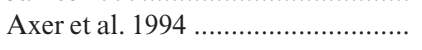 & 6114 & 4.39 & 1.05 & -1.80 \\
\hline \multicolumn{5}{|c|}{ CS 22966-043 } \\
\hline 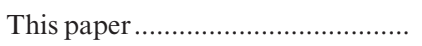 & 7400 & 3.50 & 2.85 & -1.91 \\
\hline Preston \& Sneden $2000 \ldots \ldots \ldots \ldots \ldots \ldots . . . . .$. & 7300 & 3.70 & 3.00 & -1.96 \\
\hline \multicolumn{5}{|c|}{ CS 22941-012 } \\
\hline 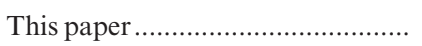 & 7200 & 4.60 & 2.40 & -2.01 \\
\hline Preston \& Sneden $2000 \ldots \ldots \ldots \ldots \ldots \ldots . . . . . .$. & 7200 & 4.20 & 2.50 & -2.03 \\
\hline \multicolumn{5}{|c|}{ G84-29 } \\
\hline 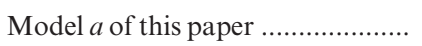 & 6000 & 2.80 & 1.10 & -2.97 \\
\hline 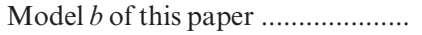 & 6330 & 3.55 & 1.40 & -2.75 \\
\hline Carretta et al. $2002 \ldots \ldots \ldots \ldots . . .$. & 6355 & 4.00 & 1.42 & -2.66 \\
\hline 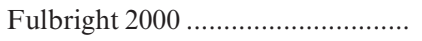 & 6075 & 3.80 & $\ldots$ & -2.84 \\
\hline 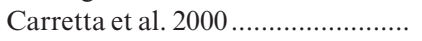 & 6224 & 3.57 & 2.10 & -2.73 \\
\hline Thévenin 1998....................... & 6246 & 3.50 & $\ldots$ & -2.80 \\
\hline 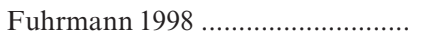 & 6330 & 3.55 & 1.4 & -2.74 \\
\hline Axer et al. $1994 \ldots \ldots \ldots \ldots$ & 6264 & 3.72 & $\ldots$ & -2.36 \\
\hline 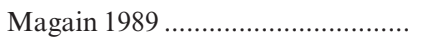 & 6110 & 3.20 & $\ldots$ & -2.98 \\
\hline \multicolumn{5}{|c|}{$\mathrm{BD}+24^{\circ} 1676$} \\
\hline 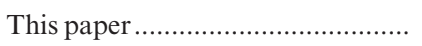 & 6250 & 3.45 & 1.20 & -2.49 \\
\hline 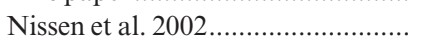 & 6251 & 3.81 & 1.50 & -2.41 \\
\hline 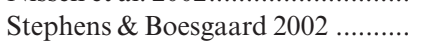 & 6136 & 3.54 & 1.59 & -2.55 \\
\hline 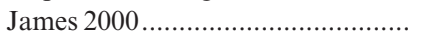 & 6200 & 3.70 & 1.55 & -2.52 \\
\hline
\end{tabular}

the same behavior in the Fe peak elements from Ti through Ga. Specifically, BD $+80^{\circ} 245$ exhibits a low average iron peak abundance with respect to iron, -0.2 to -0.3 below the scaled solar metallicity, whereas in the other two stars these elements are enhanced relative to the scaled solar metallicity ( 0.7-0.8 for CS 22966-043; 0.5 for G4-36).

\subsection{Special Notes concerning $\mathrm{Al}, \mathrm{Mn}, \mathrm{Ga}, \mathrm{Ge}$, and $\mathrm{Ba}$}

Aluminum $(Z=13)$.-With one exception, the results of the abundance analysis employing the EWs given in Table 2 are presented in Table 5, along with the standard deviation of the abundances found for multiple lines of each ion. In the case of Al, we measured EWs for both $\lambda \lambda 3944,3961$ resonance lines. However, the $\mathrm{Al}$ I $\lambda 3944$ line is affected by $\mathrm{CH}$ blending (Arpigny \& Magain 1983). We provide the EW information for the $\mathrm{Al}$ I $\lambda 3944$ line in Table 2 but exclude the abundance derived for that line from both the summary table and the figures employing the derived $\mathrm{Al}$ abundances.

Manganese $(Z=25)$. - In the case of the odd- $Z$ element $\mathrm{Mn}$, some spectral lines possess significant hyperfine structure (hfs), splitting absorption lines into multiple compo- nents. The multicomponent structure permits the line to grow to a greater strength before saturating. Without accounting for hfs, the abundance derived from a given line can be overestimated, sometimes severely (see, e.g., Gratton 1989; McWilliam et al. 1995a; Ryan et al. 1996; Prochaska $\&$ McWilliam 2000). In addition to performing single-line Mn analyses, we also computed blended-line EWs incorporating the hfs information produced by Kurucz \& Bell (1995). ${ }^{12}$ Incorporating hfs information decreased the Mn abundances derived for our sample by $0.01-0.18$ dex (the largest effect was for G4-36) and the standard deviation of the mean values by up to 0.12 dex (in the case of BD $+80^{\circ} 245$ ). Where stated or shown, all tables and figures display the $[\mathrm{Mn} / \mathrm{Fe}]$ ratio results obtained by taking hfs information into account.

Gallium $(Z=31)$.- Since the ratio of $[\mathrm{Zn} / \mathrm{Fe}]$ was found to be anomalously high in CS 22966-043 and G4-36, we also investigated the abundances of nearby elements with slightly higher atomic numbers. While $\mathrm{Ga}$ II has been studied in the class of objects known as "chemically peculiar stars" (see, e.g., Nielsen, Karlsson, \& Wahlgren 2000), the only Ga feature that could be detected in our spectrum is the line at $4172.0 \AA$ from the neutral species. The absorption lies on the wing of a blended feature of Ti and Fe. Thus, in addition to the EW analysis, we performed spectrum syntheses to derive abundances for Ga. The results of these computations are illustrated in Figure 6, where we display the syntheses derived for effectively no gallium contribution, a value close to the scaled solar contribution, and enhanced gallium abundances, superposed on the observed spectrum.

12 The hfs lists are available at http://kurucz.harvard.edu/linelists/ gfhyperall.

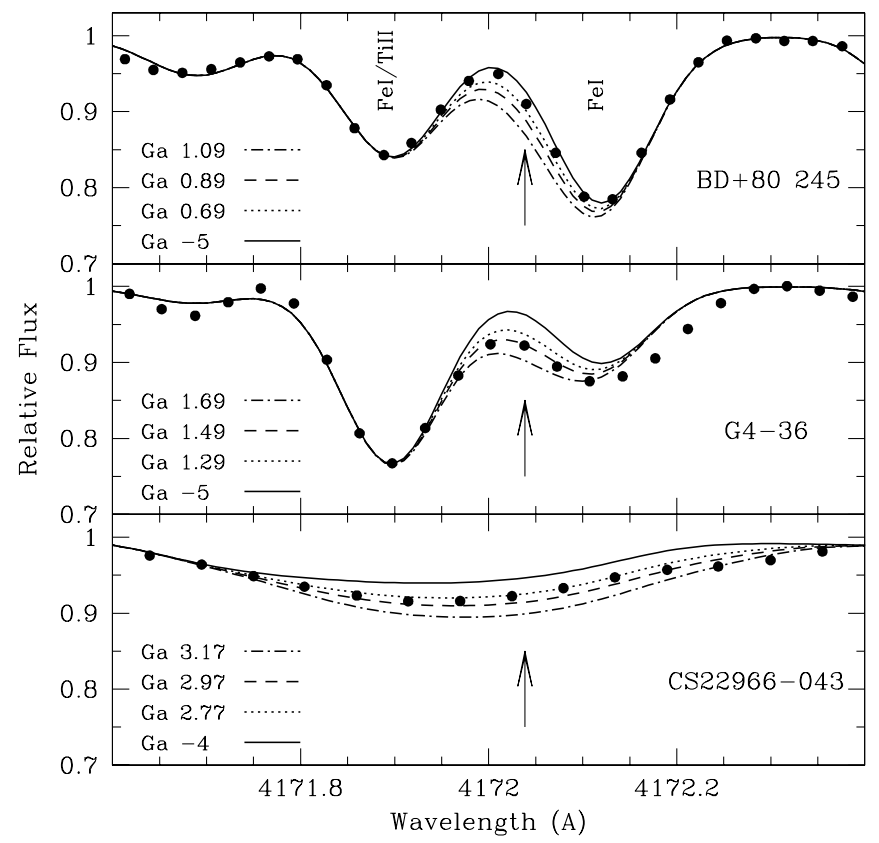

FIG. 6.-Syntheses of the gallium feature at $4172.039 \AA$ A . Filled circles denote the observed data; lines designate syntheses. Stated in each panel are the $\log \epsilon(\mathrm{Ga})$ abundances assumed in the syntheses $($ at $[\mathrm{Fe} / \mathrm{H}] \simeq-2$, the scaled solar value is 0.88 ). Also indicated are the key elemental features blended with Ga. [See the electronic edition of the Journal for a color version of this figure.] 
TABLE 5

Derived Abundances

\begin{tabular}{|c|c|c|c|c|c|c|c|c|}
\hline Element & $\mathrm{BD}+80^{\circ} 245$ & G4-36 & CS 22966-043 & CS 22941-012 & $\Delta \mathrm{CS}$ & $\mathrm{BD}+24^{\circ} 1676$ & G84-29a & G84-29b \\
\hline$\left[\mathrm{Fe}_{\mathrm{I}} / \mathrm{H}\right] \ldots \ldots \ldots \ldots \ldots$ & -2.09 & -1.93 & -1.91 & -2.02 & +0.11 & -2.50 & -2.98 & -2.76 \\
\hline 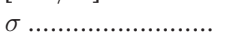 & 0.11 & 0.11 & 0.15 & 0.13 & $\ldots$ & 0.20 & 0.11 & 0.12 \\
\hline 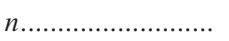 & 212 & 160 & 55 & 69 & $\ldots$ & 66 & 38 & 38 \\
\hline$[\mathrm{Fe}$ II $/ \mathrm{H}] \ldots \ldots \ldots \ldots$. & -2.04 & -1.95 & -1.91 & -2.00 & +0.09 & -2.48 & -2.96 & -2.74 \\
\hline$\sigma \ldots \ldots \ldots \ldots \ldots \ldots \ldots \ldots$ & 0.11 & 0.11 & 0.16 & 0.12 & $\ldots$ & 0.15 & 0.12 & 0.07 \\
\hline 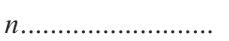 & 15 & 20 & 10 & 11 & $\ldots$ & 9 & 5 & 5 \\
\hline $\log \epsilon(\mathrm{Li}) \ldots \ldots \ldots \ldots \ldots$ & 1.44 & 2.35 & $\ldots$ & $\ldots$ & $\ldots$ & 2.45 & $<2.35$ & $<2.58$ \\
\hline$\sigma$ & $\ldots$ & $\ldots$ & $\ldots$ & $\ldots$ & $\ldots$ & $\ldots$ & $\ldots$ & $\ldots$ \\
\hline 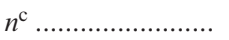 & 1 & 1 & $\ldots$ & $\ldots$ & $\ldots$ & 1 & : & : \\
\hline$[\mathrm{O} \text { I }]^{\mathrm{d}} \ldots \ldots \ldots \ldots \ldots \ldots$ & $<+0.84$ & +0.30 & $<+2.37$ & $<+2.71$ & $\ldots$ & +0.87 & $<+0.84$ & $<+0.66$ \\
\hline 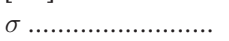 & $\ldots$ & 0.07 & $\ldots$ & $\ldots$ & $\ldots$ & 0.16 & $\ldots$ & $\ldots$ \\
\hline 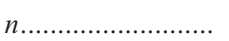 & : & 3 & : & : & $\ldots$ & 3 & : & : \\
\hline$[\mathrm{Na}$ I $] \ldots \ldots \ldots \ldots \ldots \ldots$ & -0.41 & -0.28 & -0.64 & -0.08 & -0.56 & -0.20 & -0.21 & -0.26 \\
\hline$\sigma \ldots \ldots \ldots \ldots \ldots \ldots \ldots \ldots$ & 0.01 & 0.08 & 0.16 & 0.03 & $\ldots$ & 0.05 & 0.02 & 0.00 \\
\hline 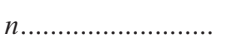 & 2 & 4 & 2 & 2 & $\ldots$ & 2 & 2 & 2 \\
\hline$[\mathrm{Mg} \mathrm{I}] \ldots \ldots \ldots \ldots \ldots \ldots$ & -0.22 & -0.19 & -0.65 & +0.27 & -0.92 & +0.49 & +0.59 & +0.40 \\
\hline$\sigma \ldots \ldots \ldots \ldots \ldots \ldots \ldots \ldots$ & 0.11 & 0.14 & 0.15 & 0.12 & $\ldots$ & 0.24 & 0.28 & 0.20 \\
\hline 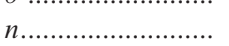 & 7 & 10 & 2 & 4 & $\cdots$ & 3 & 2 & 2 \\
\hline$[\mathrm{Al}$ I $] \ldots \ldots \ldots \ldots \ldots \ldots$ & -1.33 & -1.44 & $<-0.96$ & -0.47 & $<-0.35$ & $<+1.08$ & $\ldots$ & $\ldots$ \\
\hline$\sigma \ldots \ldots \ldots \ldots \ldots \ldots \ldots \ldots$ & $\ldots$ & $\ldots$ & $\ldots$ & 0.19 & $\ldots$ & $\ldots$ & $\ldots$ & $\ldots$ \\
\hline 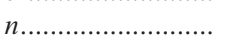 & 1 & 1 & : & 2 & : & : & $\ldots$ & $\ldots$ \\
\hline$[\mathrm{Si}$ I $] \ldots \ldots \ldots \ldots \ldots \ldots \ldots$ & -0.11 & -0.26 & -0.97 & +0.11 & -1.08 & $<+0.78$ & $\ldots$ & $\ldots$ \\
\hline 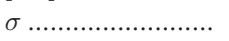 & $\ldots$ & $\ldots$ & $\ldots$ & $\ldots$ & $\ldots$ & $\ldots$ & $\ldots$ & $\ldots$ \\
\hline п & 1 & 1 & 1 & 1 & $\ldots$ & : & $\ldots$ & $\ldots$ \\
\hline$\left[\mathrm{K}_{\mathrm{I}}\right] \ldots \ldots \ldots \ldots \ldots \ldots \ldots$ & -0.23 & $<+0.15$ & $\ldots$ & $\ldots$ & $\ldots$ & $<+0.87$ & $\ldots$ & $\ldots$ \\
\hline$\sigma \ldots \ldots \ldots \ldots \ldots \ldots \ldots \ldots \ldots \ldots \ldots \ldots \ldots$ & $\ldots$ & $\ldots$ & $\ldots$ & $\ldots$ & $\ldots$ & $\ldots$ & $\ldots$ & $\ldots$ \\
\hline 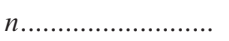 & 1 & : & $\ldots$ & $\ldots$ & $\ldots$ & : & $\ldots$ & $\ldots$ \\
\hline 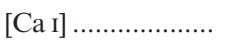 & -0.18 & -0.21 & -0.24 & +0.49 & -0.73 & +0.43 & +0.45 & +0.37 \\
\hline 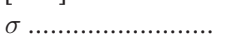 & 0.13 & 0.11 & $\ldots$ & 0.11 & $\ldots$ & 0.09 & 0.08 & 0.09 \\
\hline 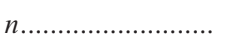 & 13 & 21 & 1 & 9 & $\ldots$ & 14 & 6 & 6 \\
\hline$[\mathrm{Ca}$ II $] \ldots \ldots \ldots \ldots \ldots \ldots$ & -0.16 & -0.11 & $\ldots$ & $\ldots$ & $\ldots$ & $\ldots$ & $\ldots$ & $\ldots$ \\
\hline$\sigma \ldots \ldots \ldots \ldots \ldots \ldots \ldots \ldots$ & $\ldots$ & $\ldots$ & $\ldots$ & $\ldots$ & $\ldots$ & $\ldots$ & $\ldots$ & $\ldots$ \\
\hline 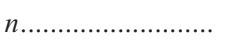 & 1 & 1 & $\ldots$ & $\ldots$ & $\ldots$ & $\ldots$ & $\ldots$ & $\ldots$ \\
\hline$[\mathrm{Sc}$ I $] \ldots \ldots \ldots \ldots \ldots \ldots$ & $\ldots$ & $\ldots$ & $<+3.49$ & $<+3.78$ & $\ldots$ & $<+3.31$ & $\ldots$ & $\ldots$ \\
\hline 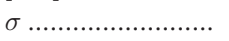 & $\ldots$ & $\ldots$ & $\ldots$ & $\ldots$ & $\ldots$ & $\ldots$ & $\ldots$ & $\ldots$ \\
\hline 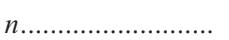 & $\ldots$ & $\ldots$ & : & : & $\ldots$ & : & $\ldots$ & $\ldots$ \\
\hline$[\mathrm{Sc}$ II $] \ldots \ldots \ldots \ldots \ldots \ldots$ & -0.42 & -0.76 & -0.76 & +0.58 & -1.34 & +0.37 & +0.16 & +0.31 \\
\hline$\sigma \quad \ldots \ldots \ldots \ldots \ldots \ldots \ldots$ & 0.10 & 0.02 & 0.02 & 0.33 & $\ldots$ & 0.14 & $\ldots$ & $\ldots$ \\
\hline 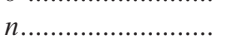 & 8 & 2 & 2 & 2 & $\ldots$ & 2 & 1 & 1 \\
\hline 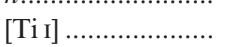 & -0.34 & +0.48 & +0.71 & +0.68 & +0.03 & +0.46 & +0.72 & +0.76 \\
\hline$\sigma$ & 0.05 & 0.08 & 0.00 & 0.16 & $\ldots$ & 0.09 & 0.13 & 0.15 \\
\hline (1) & 10 & 13 & 2 & 2 & $\begin{array}{l}\cdots \\
\cdots\end{array}$ & 4 & 4 & 4 \\
\hline$[\mathrm{Ti}$ II $] \ldots \ldots \ldots \ldots \ldots \ldots \ldots$ & -0.25 & +0.60 & +0.49 & +0.56 & -0.07 & +0.34 & +0.50 & +0.55 \\
\hline$\sigma \ldots \ldots \ldots \ldots \ldots \ldots \ldots \ldots \ldots$ & 0.08 & 0.13 & 0.10 & 0.13 & $\ldots$ & 0.15 & 0.09 & 0.09 \\
\hline 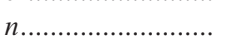 & 19 & 25 & 8 & 6 & $\ldots$ & 6 & 3 & 3 \\
\hline$\left[\mathrm{V}_{\mathrm{I}}\right] \ldots \ldots \ldots \ldots \ldots \ldots$ & -0.39 & +0.32 & $<+1.23$ & $<+1.11$ & $\ldots$ & $<+2.16$ & $<+2.53$ & $<+2.60$ \\
\hline 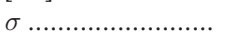 & $\ldots$ & 0.03 & $\ldots$ & $\ldots$ & $\ldots$ & $\ldots$ & $\ldots$ & $\ldots$ \\
\hline 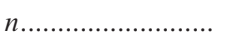 & 1 & 2 & : & : & $\ldots$ & : & : & : \\
\hline$[\mathrm{V}$ II] $\ldots \ldots \ldots \ldots \ldots \ldots \ldots$ & $<+0.44$ & +0.55 & $<+0.85$ & $<+1.22$ & $\ldots$ & $\ldots$ & $\ldots$ & $\ldots$ \\
\hline$\sigma \ldots \ldots \ldots \ldots \ldots \ldots \ldots \ldots \ldots \ldots \ldots \ldots \ldots$ & $\ldots$ & $\ldots$ & $\ldots$ & $\ldots$ & $\ldots$ & $\ldots$ & $\ldots$ & $\ldots$ \\
\hline n........................... & : & 1 & : & : & $\ldots$ & $\ldots$ & $\ldots$ & $\ldots$ \\
\hline$[\mathrm{Cr} \mathrm{I}] \ldots \ldots \ldots \ldots \ldots \ldots$ & -0.15 & +0.34 & +0.33 & +0.04 & +0.29 & +0.02 & +0.11 & +0.08 \\
\hline 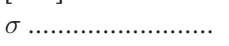 & 0.09 & 0.08 & 0.08 & 0.15 & $\ldots$ & 0.27 & 0.19 & 0.20 \\
\hline 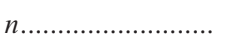 & 14 & 13 & 5 & 3 & $\ldots$ & 7 & 3 & 3 \\
\hline$[\mathrm{Cr}$ II] $] \ldots \ldots \ldots \ldots \ldots \ldots \ldots$ & +0.17 & +0.48 & +0.36 & +0.48 & -0.12 & $<+0.87$ & $\ldots$ & $\ldots$ \\
\hline$\sigma \ldots \ldots \ldots \ldots \ldots \ldots \ldots \ldots$ & 0.08 & 0.10 & 0.09 & 0.16 & $\ldots$ & $\ldots$ & $\ldots$ & $\ldots$ \\
\hline 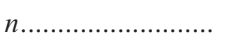 & 7 & 6 & 3 & 3 & $\ldots$ & : & $\ldots$ & $\ldots$ \\
\hline$[\mathrm{Mn}$ I $] \ldots \ldots \ldots \ldots \ldots \ldots$ & -0.26 & +0.37 & +0.41 & -0.36 & +0.77 & +0.41 & +0.38 & +0.38 \\
\hline$\sigma \ldots \ldots \ldots \ldots \ldots \ldots \ldots$ & 0.08 & 0.09 & 0.12 & 0.17 & $\ldots$ & $\ldots$ & $\ldots$ & $\ldots$ \\
\hline 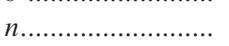 & 7 & 10 & 5 & 3 & $\ldots$ & 1 & 1 & 1 \\
\hline$[\mathrm{Co}$ I $] \ldots \ldots \ldots \ldots \ldots \ldots \ldots$ & -0.18 & +0.33 & $<+0.55$ & $<+0.29$ & $\ldots$ & $\ldots$ & $\ldots$ & $\ldots$ \\
\hline$\sigma$ & 0.10 & 0.20 & $\ldots$ & $\ldots$ & $\ldots$ & $\ldots$ & $\ldots$ & $\ldots$ \\
\hline 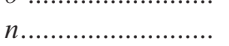 & 4 & 3 & : & : & $\cdots$ & $\cdots$ & $\cdots$ & $\cdots$ \\
\hline$[\mathrm{Ni}$ I $] \ldots \ldots \ldots \ldots \ldots \ldots \ldots$ & -0.09 & +0.48 & +0.54 & +0.28 & +0.26 & +0.30 & +0.45 & +0.42 \\
\hline$\sigma \ldots \ldots \ldots \ldots \ldots \ldots \ldots \ldots$ & 0.09 & 0.11 & 0.15 & 0.13 & $\ldots$ & 0.19 & 0.38 & 0.35 \\
\hline
\end{tabular}


TABLE 5-Continued

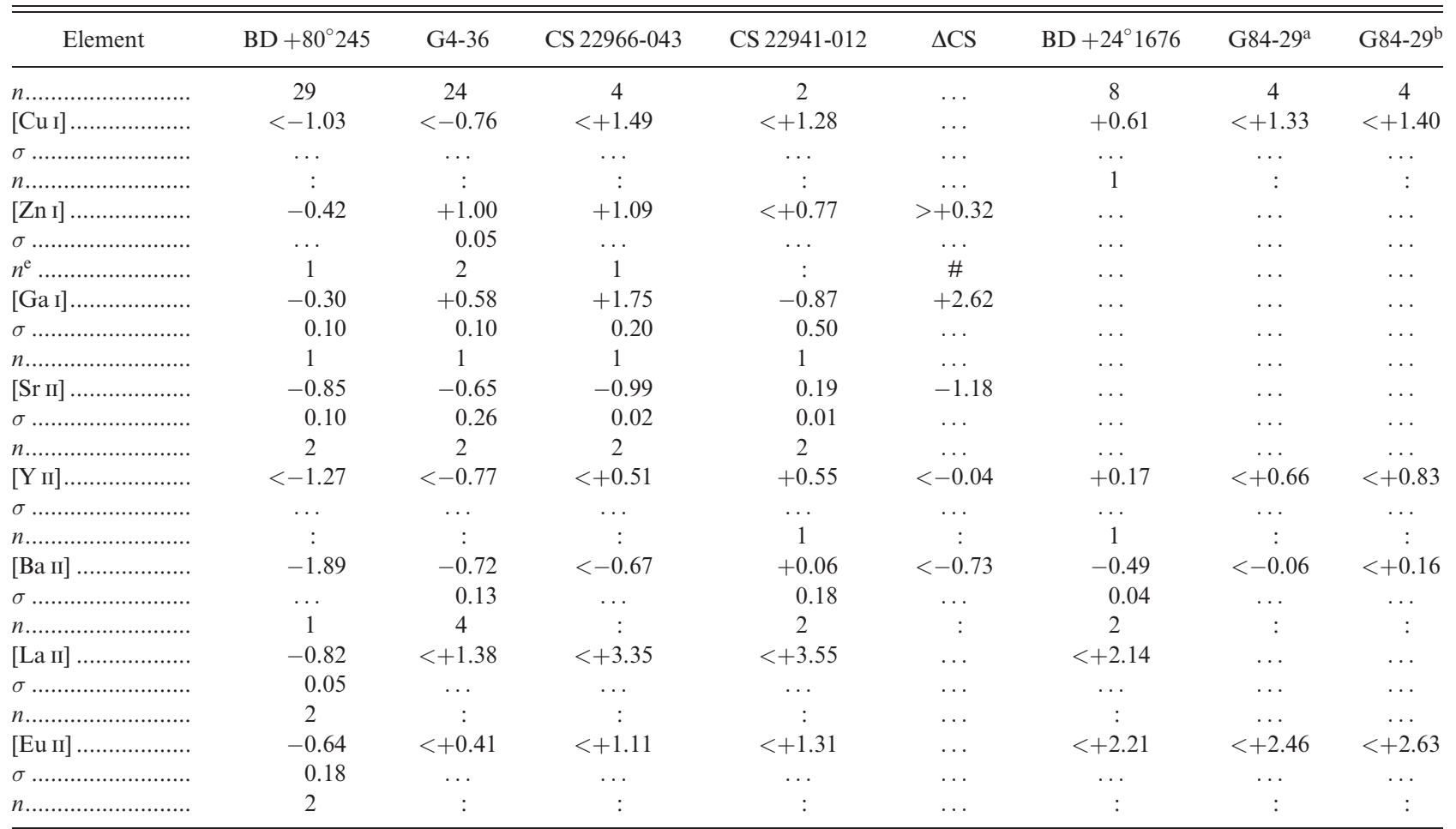

a Using model $a$ as described in Table 4

b Using model $b$ as described in Table 4.

c Upper limit on abundance denoted by a colon in place of the number of lines used in the analysis.

d [O I] denotes [O I/Fe II]. The remaining elements show the logarithmic ratio of a given elemental abundance with respect to the associated species of iron, e.g., [Na I] for [Na I/Fe I] and [Eu II] for [ $\mathrm{Eu}$ II/ $/ \mathrm{Fe}$ II].

e Lower limit on abundance difference denoted by "\#" in place of the number of lines used in the analysis.

The ability to synthesize any spectral region successfully is limited by the accuracy of the atomic parameters of the known features in the vicinity. While only $\mathrm{Ti}$ and $\mathrm{Fe}$ are known to have sufficiently sizeable contributions near the $\mathrm{Ga}$ feature, there exists the possibility that the detected feature in our spectra is a result of some other unidentified element and not due to enhanced Ga. The error bars associated with the $\mathrm{Ga}$ abundance we derived are those that are based on the assumption that $\mathrm{Ga}$, and only $\mathrm{Ga}$, significantly contributes to the blend along with $\mathrm{Ti}$ and $\mathrm{Fe}$.

The error bars on the derived ratio for CS 22966-043 denote the limits of the $\mathrm{Ga}$ abundance required to model the asymmetry of the unresolved blend. The apparent rotational velocity of CS 22966-043, $v_{\text {rot }} \sin i \approx 20 \mathrm{~km} \mathrm{~s}^{-1}$, makes it difficult to disentangle the contributions of each element. Some of the observed asymmetry may result from the pulsations of the star itself, as a result of the difference in the line-of-sight radial motion across the face of the star. Because the $\sim 90$ spectra were combined without regard to the particular phases at which the individual integrations were obtained, some pulsation phases may be more heavily weighted than others, and thus what we attribute to Ga may be over- or understated. The asymmetry in the blended feature is not completely modeled by the spectrum synthesis. In the most conservative case, the presence of Ga in CS 22966043 is an intriguing possibility worthy of further attention.

Germanium $(Z=32)$. - Since $\mathrm{Zn}$ and $\mathrm{Ga}$ were found to be enhanced in both G4-36 and CS 22966-043, we sought features of $\mathrm{Ge}$, the next higher atomic numbered element. Moore, Minnaert, \& Houtgast (1966) identify Ge I at $4226.568 \AA$ and another blended with Co at $4685.854 \AA$. Both Ge lines have good atomic parameters (Biémont et al. 1999). Unfortunately, the features are too weak to be detected in our data and are blended with much stronger lines.

Barium $(Z=56)$. - $\mathrm{Ba}$ lines possess both $\mathrm{hfs}$ and isotopic subcomponents. In addition to performing singleline analyses, we also computed blended-line EWs incorporating the hfs and isotope information from the $\mathrm{Ba}$ II line lists of McWilliam (1998). In the case of BD $+80^{\circ} 245$, for example, a single-line analysis yields $\log \epsilon(\mathrm{Ba})=-1.80$; assuming a solar abundance ratio distribution among the ${ }^{134-138} \mathrm{Ba}$ isotopes yields $\log \epsilon(\mathrm{Ba})=$ -1.83 ; and assuming an $r$-process ratio distribution among the isotopes yields $\log \epsilon(\mathrm{Ba})=-1.85$. For the slightly stronger features in G4-36, we derive $\log \epsilon(\mathrm{Ba})=$ $-0.51,-0.54$, and -0.56 , respectively. In addition, in the case of the upper limit we derive for CS 22966-043, $\log \epsilon(\mathrm{Ba})<-0.44,-0.45$, and -0.45 , respectively. It is clear that the differences in all cases are slight. This is to be expected; our program stars are all neutron-capturepoor and do not possess strong $\mathrm{Ba}$ II features. Where stated or shown, all tables and figures display the $[\mathrm{Ba} / \mathrm{Fe}]$ ratio results obtained by assuming the solar abundance ratios among the $\mathrm{Ba}$ isotopes in the calculations of the blended-line EW computations. We return to 
the issue of choosing between $r$-only and $r+s$ isotope distributions in $\S 4.2 .4$.

\subsection{Comparisons with Other Studies}

In Table 4 we summarized the stellar parameters adopted in our study, along with those derived in others. In Tables 6 , 7 , and 8 we compare our abundance results against those same studies and include from the literature the estimated error of the derived abundances (where indicated for individual stellar abundances) and the number of lines used in each analysis. In the remainder of this section we investigate the differences between these studies. Where there are few previous analyses, or where there is little disagreement between the results from various groups, discussion is brief. Instead, we focus attention on more difficult cases, specifically cases in which various studies yield results that are in poor agreement.

\subsection{1. $B D+80^{\circ} 245$}

Column (1) of Table 6 shows the elemental ratios included in this study; columns (2)-(4), (5)-(7), (8)-(10), (11)-(13), and (14)-(16) show the abundance, $\sigma$, and number of lines used to derive the abundances in five studies: the present one, Carney et al. (1997), Fulbright (2000), James (2000), and Stephens \& Boesgaard (2002). The derived abundances presented in Table 6 are all in reasonable agreement, despite the existence of a $344 \mathrm{~K}$ range in adopted temperatures as shown in Table 4. All results agree that this star has $[\mathrm{Fe} / \mathrm{H}] \simeq-2.0$ and ratios of $[\mathrm{Na} / \mathrm{Fe}],[\mathrm{Mg} / \mathrm{Fe}]$, $[\mathrm{Ca} / \mathrm{Fe}],[\mathrm{Ti} / \mathrm{Fe}],[\mathrm{Mn} / \mathrm{Fe}]$, and $[\mathrm{Co} / \mathrm{Fe}]$ well below the scaled solar value, as well as extremely low abundance ratios of $[\mathrm{Zn} / \mathrm{Fe}]$, [Sr/Fe], [Ba/Fe], and [Eu/Fe].

$$
\text { 3.2.2. } G 4-36
$$

Results from this study and those of James (2000) are presented in Table 7. For all but two of the species in common, we are in excellent accord. While both analyses find unusually high $[(\mathrm{Mn}, \mathrm{Co}) / \mathrm{Fe}]$ ratios, the ones reported by James are significantly higher. The $[\mathrm{Mn} / \mathrm{Fe}]$ difference can be explained by accounting for hfs information in our analysis that resulted in a lower abundance ratio by 0.18 dex than we would otherwise have derived. In the case of the $[\mathrm{Co} / \mathrm{Fe}]$ ratio, the result reported by James was based on a single line with an excitation potential of $1.05 \mathrm{eV}$. Lowering the excitation potential to correspond with the value we employed in this study lowers the $[\mathrm{Co} / \mathrm{Fe}]$ by $0.46 \mathrm{dex}$.

Our stellar parameters are in very good agreement; in both studies, they were derived by employing spectroscopic constraints. As a consistency check of the $\log g$ value we derived by satisfying the iron ionization equilibrium constraint, we employed the Hipparcos (ESA 1997) parallax of $\pi=4.16 \pm 3.37$ mas to calculate a gravity of $4.72_{-1.44}^{+0.72}$ via the following relation derived from fundamental considerations:

$$
\begin{aligned}
\log \left(\frac{g_{*}}{g_{\odot}}\right)= & \log \left(\frac{M_{*}}{M_{\odot}}\right)+4 \log \left(\frac{T_{\text {eff } *}}{T_{\text {eff } \odot}}\right)+2 \log \pi \\
& +0.4\left(V+\mathrm{BC}_{V}-A_{V}\right)+0.13
\end{aligned}
$$

assuming that the star is $0.8 M_{\odot}$ and suffers from a reddening of $E(B-V)=0.04$. Unfortunately, none of our stars have a measured parallax greater than 8 mas; for some of our stars, the stated errors are almost as large as the parallaxes themselves. Nonetheless, for G4-36, we find that the $\log g$ value derived from the Hipparcos parallax is, to within the errors, in agreement with that derived spectroscopically.

Aside from a few photometric estimates of temperature and metallicity, we found only one reference in the literature prior to James' discovery of the unusual nature of G4-36 that included this object in a spectroscopic analysis. Axer, Fuhrmann, \& Gehren (1994) derive values for $\left(T_{\text {eff }}, \log g\right.$, $[\mathrm{Fe} / \mathrm{H}])$ of $(6114 \mathrm{~K}, 4.39,-1.51)$ via a best fit to Balmer line profiles and ionization equilibrium constraints. To test the dependence of the abundances we derived on the choice of stellar parameters, we repeated the analysis of G4-36 using the Axer et al. (1994) parameters. We include the results in column (8) of Table 7. For both sets of stellar parameters, the spectroscopic constraints for $T_{\text {eff }}$ (excitation potential), $\log g$ (ionization equilibrium), and $\xi_{t}$ (equivalent width) are met equally well (to within the errors of the abundance determinations). The only differences of any significance between the results employing the parameters adopted in our study and those from using the Axer et al. (1994) model are that, using our parameters, we derive a slightly higher $\left[\mathrm{O}_{\mathrm{I}} / \mathrm{Fe} \mathrm{II}\right]$ ratio and a lower iron abundance. The remaining abundances, with respect to the derived iron abundance, are very robust. Anomalous $[\mathrm{X} / \mathrm{Fe}]$ values remain anomalous and are not a result of choices in the model parameters.

In our analysis of this star, there exist a few elemental abundances that depend on the EW of only one feature: $\mathrm{Si}, \mathrm{K}$, and $\mathrm{Cu}$. Furthermore, since these elements were not included in James' analysis, there is no independent comparison available for the abundances we derived. For $\mathrm{K}$ and $\mathrm{Cu}$ in this star, we determined only upper limits. In the case of $\mathrm{Si}$, we used the $\mathrm{Si}$ I $\lambda 3905.53$ feature employed in previous studies by our group (see, e.g., Cowan et al. 2002). The red wing of this feature is blended with $\mathrm{CH}$ and another unidentified feature at $3905.769 \AA$ (Moore et al. 1966). However, the EW we adopted is based on double that of the Gaussian profile fit to the clean blue side of the feature.

\subsubsection{CS 22966-043}

In Table 8, columns (2)-(4) and (5)-(7) show the abundance, $\sigma$, and number of lines used to derive the abundances of CS 22966-043 in the present study and that of Preston \& Sneden (2000). Columns (8)-(10) and (11)-(13) show the same quantities for CS 22941-012, the adopted comparison BMP star. Between the two studies, in both stars all but the abundances of two of the elements agree to within $1 \sigma$. In the case of the $[\mathrm{Sc} / \mathrm{Fe}]$ ratio in CS 22966-043, the agreement is within $3 \sigma$, with both studies finding very low values. In addition, in the case of the $[\mathrm{Sr} / \mathrm{Fe}]$ ratio, the agreement is within $2 \sigma$ for both stars. Both cases can be understood as being due to the differences in the adopted stellar parameters (as shown in Table 4), largely being driven by the difference in $\log g$. The present study not only confirms the results reported by Preston \& Sneden (2000) but expands the number of elements for which abundances or upper limits have been derived. Of particular interest are the enhanced values of Ni, Zn, and possibly Ga observed in CS 22966-043, which will be discussed further in $\S 3.4$ and subsequent sections.

\subsection{4. $G 84-29$}

The literature regarding this relatively bright metal-poor star shows poor agreement in the adopted stellar parameters 


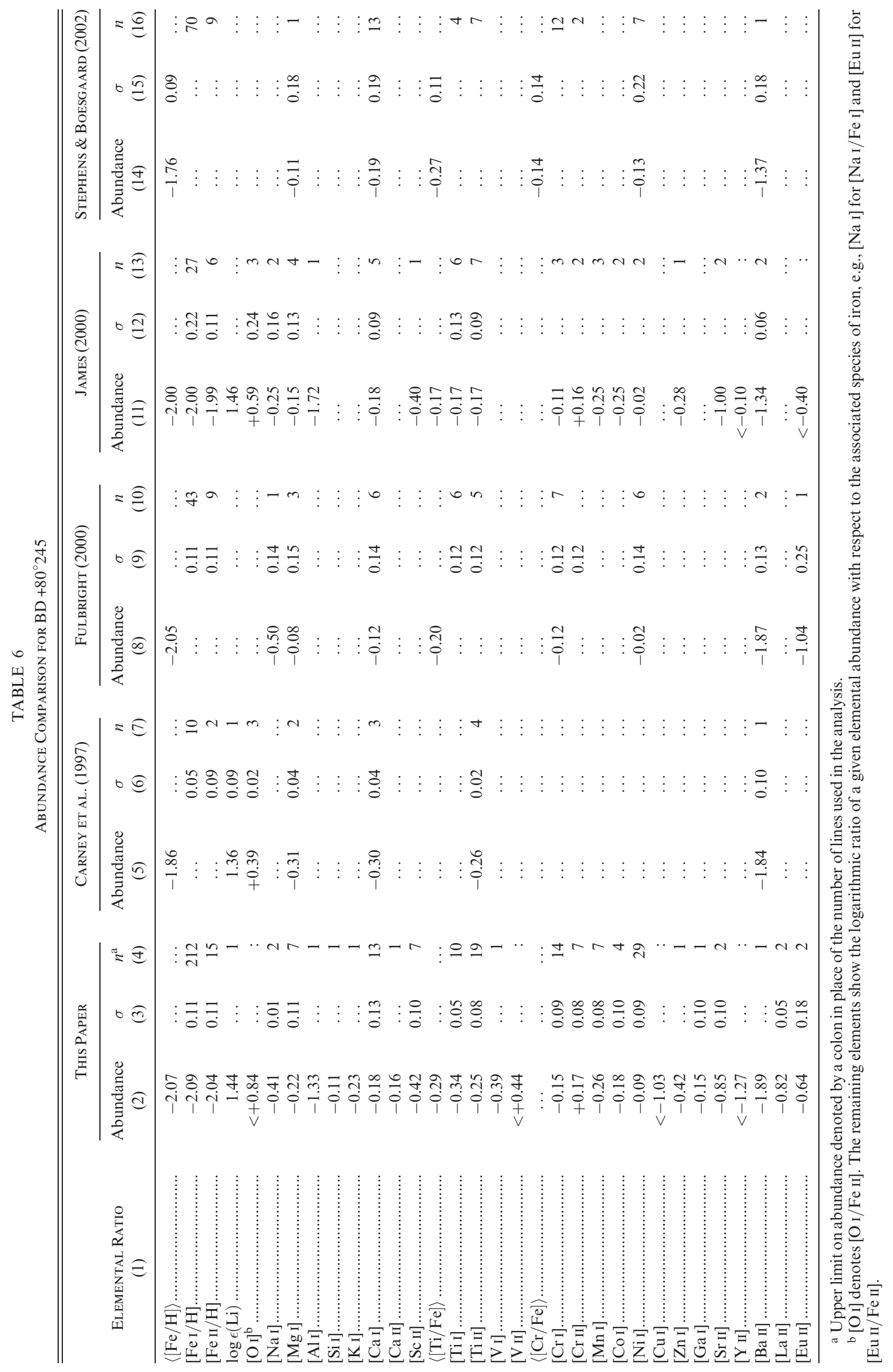


TABLE 7

Abundance Comparison for G4-36

\begin{tabular}{|c|c|c|c|c|c|c|c|}
\hline \multirow[b]{2}{*}{$\begin{array}{c}\text { ELEMENTAL RATIO } \\
\text { (1) }\end{array}$} & \multicolumn{3}{|c|}{ THIS PAPER } & \multicolumn{3}{|c|}{ JAMES (2000) } & \multirow{2}{*}{$\begin{array}{c}\text { Stellar Model Parameters } \\
\text { From AxER ET AL. (1994) } \\
\text { (Our EWs) } \\
(8)\end{array}$} \\
\hline & $\begin{array}{l}\text { Abundance } \\
\text { (2) }\end{array}$ & $\begin{array}{c}\sigma \\
(3)\end{array}$ & $\begin{array}{l}n^{\mathrm{a}} \\
(4)\end{array}$ & $\begin{array}{l}\text { Abundance } \\
\text { (5) }\end{array}$ & $\begin{array}{c}\sigma \\
(6)\end{array}$ & $\begin{array}{l}n \\
(7)\end{array}$ & \\
\hline$\langle[\mathrm{Fe} / \mathrm{H}]\rangle \ldots \ldots \ldots \ldots \ldots$ & -1.94 & & $\ldots$ & -1.96 & $\ldots$ & $\ldots$ & -1.80 \\
\hline$\left[\mathrm{Fe}_{\mathrm{I}} / \mathrm{H}\right] \ldots \ldots \ldots \ldots \ldots \ldots \ldots \ldots \ldots \ldots$ & -1.93 & 0.11 & 160 & -1.95 & 0.05 & 29 & -1.81 \\
\hline$[\mathrm{Fe}$ II $/ \mathrm{H}] \ldots \ldots \ldots \ldots \ldots$ & -1.95 & 0.11 & 20 & -1.97 & 0.08 & 7 & -1.79 \\
\hline $\log \epsilon(\mathrm{Li}) \ldots \ldots \ldots \ldots \ldots \ldots$ & 2.39 & $\ldots$ & 1 & 2.33 & $\ldots$ & 1 & 2.51 \\
\hline$[\mathrm{O}]^{\mathrm{b}}$ & +0.30 & 0.07 & 3 & +0.28 & 0.07 & 3 & +0.19 \\
\hline$[\mathrm{Na}$ I $] \ldots \ldots \ldots \ldots \ldots \ldots \ldots \ldots \ldots \ldots \ldots \ldots \ldots$ & -0.28 & 0.08 & 4 & -0.08 & 0.12 & 4 & -0.36 \\
\hline 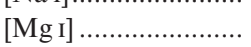 & -0.19 & 0.14 & 10 & -0.12 & 0.20 & 6 & -0.29 \\
\hline$[\mathrm{Al}$ I] $\ldots \ldots \ldots \ldots \ldots \ldots \ldots \ldots \ldots \ldots \ldots$ & -1.44 & $\ldots$ & 1 & -1.53 & $\ldots$ & 1 & -1.17 \\
\hline$[\mathrm{Si}$ I] $] \ldots \ldots \ldots \ldots \ldots \ldots \ldots \ldots \ldots \ldots \ldots \ldots \ldots$ & -0.26 & $\ldots$ & 1 & $\ldots$ & $\ldots$ & $\ldots$ & -0.30 \\
\hline$[\mathrm{K}$ I $] \ldots \ldots \ldots \ldots \ldots \ldots \ldots \ldots \ldots \ldots \ldots$ & $<+0.15$ & $\ldots$ & : & $\ldots$ & $\ldots$ & $\ldots$ & $\ldots$ \\
\hline 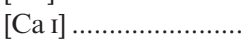 & -0.21 & 0.11 & 21 & -0.12 & 0.16 & 5 & -0.26 \\
\hline$[\mathrm{Ca}$ II $] \ldots \ldots \ldots \ldots \ldots \ldots$ & -0.11 & & 1 & $\ldots$ & $\ldots$ & $\ldots$ & -0.19 \\
\hline$[\mathrm{Sc}$ II $] \ldots \ldots \ldots \ldots \ldots$ & -0.76 & 0.02 & 2 & -0.46 & $\ldots$ & 1 & -0.70 \\
\hline$\langle[\mathrm{Ti} / \mathrm{Fe}]\rangle \ldots \ldots \ldots \ldots \ldots$ & +0.54 & $\ldots$ & $\ldots$ & +0.52 & $\ldots$ & $\ldots$ & +0.58 \\
\hline 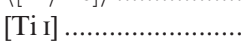 & +0.48 & 0.08 & 13 & +0.53 & 0.05 & 6 & +0.50 \\
\hline$[$ [Ti II $] \ldots \ldots \ldots \ldots \ldots \ldots \ldots$ & +0.60 & 0.13 & 25 & +0.51 & 0.04 & 7 & +0.65 \\
\hline$\left[\mathrm{V}_{\mathrm{I}} \mathrm{\ldots}\right] \ldots \ldots \ldots \ldots \ldots$ & +0.32 & 0.03 & 2 & $\ldots$ & $\ldots$ & $\ldots$ & +0.33 \\
\hline$[\mathrm{V}$ II $] \ldots \ldots \ldots \ldots \ldots \ldots \ldots \ldots \ldots \ldots \ldots \ldots \ldots \ldots$ & +0.55 & & 1 & $\ldots$ & $\ldots$ & $\ldots$ & +0.59 \\
\hline 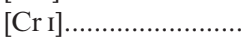 & +0.34 & 0.08 & 13 & +0.25 & 0.14 & 3 & +0.34 \\
\hline$[\mathrm{Cr}$ II] $] \ldots \ldots \ldots \ldots \ldots \ldots \ldots \ldots \ldots \ldots \ldots$ & +0.48 & 0.10 & 6 & +0.19 & 0.05 & 2 & +0.46 \\
\hline 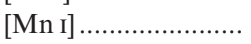 & +0.37 & 0.09 & 10 & +0.52 & 0.07 & 3 & +0.35 \\
\hline$[\mathrm{Co}$ I $] \ldots \ldots \ldots \ldots \ldots \ldots$ & +0.33 & 0.20 & 3 & +0.70 & $\ldots$ & 1 & +0.37 \\
\hline$[\mathrm{Ni}$ I $] \ldots \ldots \ldots \ldots \ldots \ldots$ & +0.48 & 0.11 & 24 & +0.54 & 0.02 & 2 & +0.46 \\
\hline 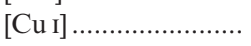 & $<-0.76$ & & : & $\ldots$ & $\ldots$ & 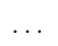 & \\
\hline$[\mathrm{Zn} \mathrm{I}] \ldots \ldots \ldots \ldots \ldots \ldots$ & +1.00 & 0.05 & 2 & +0.96 & $\ldots$ & 1 & +1.00 \\
\hline$[\mathrm{Ga}$ I $] \ldots \ldots \ldots \ldots \ldots \ldots \ldots \ldots \ldots \ldots \ldots$ & +0.64 & 0.10 & 1 & $\ldots$ & $\ldots$ & $\ldots$ & $\ldots$ \\
\hline$[\mathrm{Sr}$ II $] \ldots \ldots \ldots \ldots \ldots \ldots$ & -0.65 & 0.26 & 2 & -0.95 & $\ldots$ & 1 & -0.57 \\
\hline 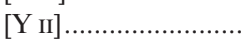 & $<-0.77$ & $\ldots$ & : & $\ldots$ & $\ldots$ & : & $\ldots$ \\
\hline$[\mathrm{Ba}$ II] $\ldots \ldots \ldots \ldots \ldots \ldots \ldots$ & -0.72 & 0.13 & 4 & -0.70 & 0.14 & 4 & -0.61 \\
\hline 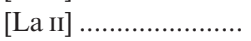 & $<+1.38$ & $\ldots$ & : & $\ldots$ & $\ldots$ & $\ldots$ & $\ldots$ \\
\hline$[\mathrm{Eu}$ II] $\ldots \ldots \ldots \ldots \ldots \ldots \ldots \ldots \ldots \ldots \ldots$ & $<+0.41$ & $\ldots$ & $:$ & $<+0.32$ & $\ldots$ & : & $\ldots$ \\
\hline
\end{tabular}

a Upper limit on abundance denoted by a colon in place of the number of lines used in the analysis.

b $[\mathrm{O}$ I] denotes [O I/Fe II]. The remaining elements show the logarithmic ratio of a given elemental abundance with respect to the associated species of iron, e.g., [Na I] for [Na I/Fe I] and [Eu II] for [Eu II/Fe II].

from study to study. Satisfying the spectroscopic constraints in excitation potential, EW, and ionization equilibrium results in relatively low values for the temperature, $\log g$, and $[\mathrm{Fe} / \mathrm{H}]$ compared to other studies as shown in Table 4. This behavior is consistent with the idea of $\mathrm{Fe}$ overionization due to non-LTE effects, which are expected to exhibit a larger effect in lower metallicity and higher temperature stars (see, e.g., Thévenin \& Idiart 1999; Korn, Shi, \& Gehren 2003; Kraft \& Ivans 2003).

To explore the effects that a hotter temperature and higher $\log g$ model would make on the derived abundances for this star, we ran an analysis with our EWs using an atmospheric model based on the Fuhrmann (1998) parameters $\left[\left(T_{\text {eff }}, \log g\right)=(6330 \mathrm{~K}, 3.45)\right.$ vs. the spectroscopically derived $(6000 \mathrm{~K}, 3.0)]$. While the derived iron abundance using the Fuhrmann parameters is a function of neither EW nor ionization state, there is a strong trend with excitation potential that indicates that the temperature is too hot by a few hundred degrees. A similar and unresolved difficulty was encountered by Carretta et al. (2002) in their analysis of HE 0132-2429 (see their Fig. $5 a$ ). In the case of G84-29, the higher temperature is closer to (1) the estimates derived employing the empirical
$T_{\text {eff-color calibrations of Alonso, Arribas, \& Martínez- }}$ Roger $(1996,1999,2001)$ and (2) the $6400 \mathrm{~K}$ temperature derived by Cohen et al. (2002) via a best fit to the $\mathrm{H} \alpha$ Balmer line profile.

In this study we will treat the derived abundances for G84-29 from both sets of models as being equally plausible in order to investigate the effects of the abundance ratio differences. In Tables 5, 9, and 12, the results from "model $a$ " refer to those obtained with stellar parameters of ( $T_{\text {eff }}, \log g$, $[\mathrm{Fe} / \mathrm{H}])$ of $(6000 \mathrm{~K}, 2.80,-2.97)$, and the results from "model $b$ " refer to those of $(6330 \mathrm{~K}, 3.50,-2.75)$. Within the errors, most of the abundance ratios relative to iron show no significant differences.

In Table 9 we also catalog the abundances found in previous studies of G84-29 along with those derived in the present study. Among previous claims are unusually low $\alpha$ element abundances. In the remainder of this subsection we give an overview of some of the differences between various studies of this star.

Thévenin \& Idiart. (1999). - In their study, input values of $\left(T_{\text {eff }}, \log g,[\mathrm{M} / \mathrm{H}], \xi_{t}\right)=(6146 \mathrm{~K}, 3.50,-2.8,1.2) \quad$ were adopted from the compilation of Thévenin (1998). Table 9 shows the abundance ratios subsequent to a straightforward 
TABLE 8

BMP Star Abundance Comparisons with Preston \& Sneden (2000)

\begin{tabular}{|c|c|c|c|c|c|c|c|c|c|c|c|c|}
\hline \multirow[b]{3}{*}{$\begin{array}{c}\text { ELEMENTAL Ratio } \\
\text { (1) }\end{array}$} & \multicolumn{6}{|c|}{ CS 22966-043 } & \multicolumn{6}{|c|}{ CS 22941-012 } \\
\hline & \multicolumn{3}{|c|}{ THIS PAPER } & \multicolumn{3}{|c|}{ Preston \& SNEdEN (2000) } & \multicolumn{3}{|c|}{ THIS PAPER } & \multicolumn{3}{|c|}{ PREston \& SNEDEN (2000) } \\
\hline & $\begin{array}{c}\text { Abundance } \\
\text { (2) }\end{array}$ & $\begin{array}{c}\sigma \\
(3)\end{array}$ & $\begin{array}{l}n^{\mathrm{a}} \\
(4)\end{array}$ & $\begin{array}{l}\text { Abundance } \\
\text { (5) }\end{array}$ & $\begin{array}{c}\sigma \\
(6)\end{array}$ & $\begin{array}{c}n \\
(7)\end{array}$ & $\begin{array}{l}\text { Abundance } \\
\text { (8) }\end{array}$ & $\begin{array}{c}\sigma \\
(9)\end{array}$ & $\begin{array}{c}n \\
(10)\end{array}$ & $\begin{array}{l}\text { Abundance } \\
\text { (11) }\end{array}$ & $\begin{array}{c}\sigma \\
(12)\end{array}$ & $\begin{array}{c}n \\
(13)\end{array}$ \\
\hline$\langle[\mathrm{Fe} / \mathrm{H}]\rangle \ldots \ldots \ldots \ldots \ldots$ & -1.91 & $\ldots$ & $\ldots$ & -1.96 & $\ldots$ & $\ldots$ & -2.01 & $\ldots$ & $\ldots$ & -2.03 & $\ldots$ & $\ldots$ \\
\hline$[\mathrm{Fe}$ I/H] $] \ldots \ldots \ldots \ldots \ldots \ldots$ & -1.91 & 0.15 & 55 & $\ldots$ & 0.05 & 26 & -2.02 & 0.13 & 69 & $\ldots$ & 0.05 & 29 \\
\hline$[\mathrm{Fe}$ II $/ \mathrm{H}] \ldots \ldots \ldots \ldots \ldots \ldots$ & -1.91 & 0.16 & 10 & $\ldots$ & 0.10 & 7 & -2.00 & 0.12 & 11 & $\ldots$ & 0.13 & 4 \\
\hline$[\mathrm{O} \text { I }]^{\mathrm{b}} \ldots \ldots \ldots \ldots \ldots \ldots \ldots$ & $<+2.37$ & $\ldots$ & : & $\ldots$ & $\ldots$ & $\ldots$ & $<+2.71$ & $\ldots$ & : & $\ldots$ & $\ldots$ & $\ldots$ \\
\hline$[\mathrm{Na} \mathrm{I}] \ldots \ldots \ldots \ldots \ldots \ldots \ldots \ldots$ & -0.64 & 0.16 & 2 & $\ldots$ & $\ldots$ & $\cdots$ & -0.08 & 0.03 & 2 & $\ldots$ & $\ldots$ & $\cdots$ \\
\hline$[\mathrm{Mg} \mathrm{I}] \ldots \ldots \ldots \ldots \ldots \ldots \ldots$ & -0.65 & 0.15 & 2 & -0.64 & 0.11 & 2 & +0.27 & 0.12 & 4 & +0.31 & 0.09 & 3 \\
\hline$[\mathrm{Al}$ I $] \ldots \ldots \ldots \ldots \ldots \ldots \ldots \ldots \ldots \ldots$ & $<-0.96$ & $\ldots$ & : & $\ldots$ & $\ldots$ & $\ldots$ & -0.47 & $\ldots$ & 1 & $\ldots$ & $\ldots$ & $\ldots$ \\
\hline 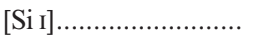 & -0.97 & $\ldots$ & 1 & $\ldots$ & $\ldots$ & $\ldots$ & +0.11 & $\ldots$ & 1 & $\ldots$ & $\ldots$ & $\ldots$ \\
\hline$[\mathrm{Ca}$ I $] \ldots \ldots \ldots \ldots \ldots \ldots \ldots \ldots \ldots \ldots$ & -0.24 & $\ldots$ & 1 & -0.35 & 0.13 & 2 & +0.49 & 0.11 & 9 & +0.45 & 0.04 & 6 \\
\hline$[\mathrm{Sc}$ II $] \ldots \ldots \ldots \ldots \ldots \ldots \ldots$ & -0.76 & 0.02 & 2 & -0.46 & 0.11 & 2 & +0.58 & 0.33 & 2 & +0.35 & 0.15 & 1 \\
\hline$[\mathrm{Ti}$ I $] \ldots \ldots \ldots \ldots \ldots \ldots \ldots \ldots$ & +0.71 & 0.00 & 2 & $\ldots$ & $\ldots$ & $\ldots$ & +0.68 & 0.16 & 2 & $\ldots$ & $\ldots$ & $\ldots$ \\
\hline$[\mathrm{Ti}$ II $] \ldots \ldots \ldots \ldots \ldots \ldots \ldots$ & +0.49 & 0.10 & 8 & +0.60 & 0.06 & 15 & +0.56 & 0.13 & 6 & +0.55 & 0.06 & 15 \\
\hline$\left[\mathrm{V}_{\mathrm{I}}\right] \ldots \ldots \ldots \ldots \ldots \ldots \ldots \ldots \ldots \ldots$ & $<+1.23$ & $\cdots$ & $:$ & $\ldots$ & $\ldots$ & $\cdots$ & $<+1.11$ & $\cdots$ & $:$ & $\ldots$ & $\ldots$ & $\cdots$ \\
\hline$\left[\mathrm{V}_{\mathrm{II}}\right] \ldots \ldots \ldots \ldots \ldots \ldots \ldots \ldots \ldots \ldots \ldots$ & $<+0.85$ & $\ldots$ & $:$ & $\ldots$ & $\ldots$ & $\ldots$ & $<+1.22$ & $\ldots$ & $:$ & $\ldots$ & $\ldots$ & $\ldots$ \\
\hline$[\mathrm{Cr}$ I $] \ldots \ldots \ldots \ldots \ldots \ldots \ldots \ldots \ldots \ldots \ldots \ldots$ & +0.33 & 0.08 & 5 & +0.37 & 0.06 & 2 & +0.04 & 0.15 & 3 & -0.07 & 0.06 & 2 \\
\hline$[\mathrm{Cr}$ II $] \ldots \ldots \ldots \ldots \ldots \ldots \ldots$ & +0.36 & 0.09 & 3 & $\ldots$ & $\ldots$ & $\ldots$ & +0.48 & 0.16 & 3 & $\ldots$ & $\ldots$ & $\cdots$ \\
\hline$[\mathrm{Mn} \mathrm{I}] \ldots \ldots \ldots \ldots \ldots \ldots \ldots$ & +0.41 & 0.12 & 5 & +0.33 & 0.15 & 2 & -0.36 & 0.17 & 3 & -0.21 & 0.15 & 2 \\
\hline$[\mathrm{Co}$ I $] \ldots \ldots \ldots \ldots \ldots \ldots \ldots \ldots$ & $<+0.55$ & $\ldots$ & : & $\ldots$ & $\ldots$ & $\ldots$ & $<+0.29$ & $\ldots$ & : & $\ldots$ & $\ldots$ & $\ldots$ \\
\hline$[\mathrm{Ni}$ I $] \ldots \ldots \ldots \ldots \ldots \ldots \ldots \ldots \ldots \ldots \ldots$ & +0.54 & 0.15 & 4 & $\cdots$ & $\ldots$ & $\cdots$ & +0.28 & 0.13 & 2 & $\cdots$ & $\cdots$ & $\cdots$ \\
\hline$[\mathrm{Cu}$ I $] \ldots \ldots \ldots \ldots \ldots \ldots \ldots \ldots$ & $<+1.49$ & $\ldots$ & $:$ & $\ldots$ & $\ldots$ & $\ldots$ & $<+1.28$ & $\ldots$ & $:$ & $\ldots$ & $\cdots$ & $\ldots$ \\
\hline$[\mathrm{Zn} \mathrm{I}] \ldots \ldots \ldots \ldots \ldots \ldots \ldots$ & +1.09 & $\ldots$ & 1 & $\ldots$ & $\ldots$ & $\ldots$ & $<+0.77$ & $\ldots$ & $:$ & $\ldots$ & $\ldots$ & $\ldots$ \\
\hline$[\mathrm{Ga}$ I $] \ldots \ldots \ldots \ldots \ldots \ldots \ldots$ & +1.75 & 0.20 & 1 & $\ldots$ & $\ldots$ & $\ldots$ & -0.87 & 0.50 & 1 & $\ldots$ & $\ldots$ & $\ldots$ \\
\hline$[\mathrm{Sr}$ II $] \ldots \ldots \ldots \ldots \ldots \ldots \ldots \ldots \ldots \ldots \ldots \ldots$ & -0.99 & 0.02 & 2 & -1.11 & 0.08 & 2 & -0.19 & 0.01 & 2 & -0.04 & 0.08 & 2 \\
\hline$[\mathrm{Y}$ II $] \ldots \ldots \ldots \ldots \ldots \ldots \ldots \ldots \ldots \ldots \ldots$ & $<+0.51$ & $\ldots$ & $:$ & $\ldots$ & $\ldots$ & $\cdots$ & +0.55 & $\cdots$ & 1 & $\ldots$ & $\ldots$ & $\cdots$ \\
\hline$[\mathrm{Ba}$ II $] \ldots \ldots \ldots \ldots \ldots \ldots \ldots \ldots \ldots \ldots$ & $<-0.67$ & $\cdots$ & $:$ & $<-0.36$ & $\ldots$ & : & +0.06 & 0.18 & 2 & +0.13 & $\cdots$ & 1 \\
\hline$[\mathrm{La}$ II $] \ldots \ldots \ldots \ldots \ldots \ldots \ldots$ & $<+3.35$ & $\ldots$ & $:$ & $\ldots$ & $\ldots$ & $\ldots$ & $<+3.55$ & $\ldots$ & : & $\ldots$ & $\ldots$ & $\ldots$ \\
\hline$[\mathrm{Eu}$ II] $] \ldots \ldots \ldots \ldots \ldots \ldots \ldots$ & $<+1.11$ & $\ldots$ & $:$ & $\cdots$ & $\cdots$ & $\cdots$ & $<+1.31$ & $\cdots$ & : & $\ldots$ & $\ldots$ & $\ldots$ \\
\hline
\end{tabular}

\footnotetext{
a Upper limit on abundance denoted by a colon in place of the number of lines used in the analysis.

${ }^{b}[\mathrm{O}$ I] denotes [O I/Fe II]. The remaining elements show the logarithmic ratio of a given elemental abundance with respect to the associated species of iron, e.g., [Na I] for [Na I/Fe I] and [Eu II] for [Eu II/Fe II].
}

revision of $\log \epsilon(\mathrm{Fe})=7.52$ from the value of 7.46 adopted by Thévenin.

Axer et al. (1994); Fuhrmann et al. (1995); Fuhrmann (1998).-Employing stellar parameters derived by Axer et al. (1994), Fuhrmann, Axer, \& Gehren (1995) report an unusually low $[\mathrm{Mg} / \mathrm{Fe}]$ ratio of $-0.28 \pm 0.11$, based on two $\mathrm{Mg}$ lines. For the same two lines, employing model $a$, we derive an abundance of $0.52 \pm 0.08$. Going to the hotter and higher gravity model $b$ drops the $[\mathrm{Mg} / \mathrm{Fe}]$ ratio by 0.19 dex, but the result is still significantly higher than the abundance derived by Fuhrmann et al. (1995). We are unable to confirm the unusually low $\alpha$ results of Fuhrmann et al. (1995).

Carretta et al. (2000, 2002).-Caretta, Gratton, \& Sneden (2000) employed stellar parameters similar to those derived by Fuhrmann (1998): $\left(T_{\text {eff }}, \log g,[\mathrm{Fe} / \mathrm{H}], \xi_{t}\right)=$ $(6224 \mathrm{~K}, 3.57,-2.73,2.10)$. Using this model, Carretta et al. (2000) found $[\mathrm{Mg} / \mathrm{Fe}]=0.41$. More recently, Carretta et al. (2002) have performed a new analysis of this object based on new data (Cohen et al. 2002). With their new model $(6355 \mathrm{~K}, 4.0,-2.66,1.42)$, they derive $[\mathrm{Mg} / \mathrm{Fe}]=$ $0.35 \pm 0.16$, in good agreement with the previous value.

Magain (1989); Zhao \& Magain (1990). - The model parameters chosen by the Magain studies are very similar to those we derived. Thus, the good agreement between the abundances of $\mathrm{Fe}, \mathrm{Na}$, and $\mathrm{Mg}$ derived in the two studies is no surprise. There are offsets in the abundances derived for $\mathrm{Ti}$ and $\mathrm{Cr}$, but these are both within $2 \sigma$. Given the good agreement with the rest of the elements in their studies, it seems likely that different choices in the adopted atomic parameters are the cause of the differences in the abundance determinations for these specific elements, for which we have no lines in common.

Fulbright $(1999,2000,2002)$.- Aside from differences in the derived metallicity and model parameters, the remainder of the abundances we derive for species in common are exceptionally good. The ratios of $[\mathrm{Na} / \mathrm{Fe}],[\mathrm{Mg} / \mathrm{Fe}]$, and $[\mathrm{Ca} / \mathrm{Fe}]$ are all within $0.02 \mathrm{dex}$, and the ratio of $[\mathrm{Cr} / \mathrm{Fe}]$ is within $0.1 \mathrm{dex}$. This agreement, in spite of the difference in stellar parameters, attests to the reliability with which our derived abundances should be viewed.

In summary, our results for G84-29 include abundances for more elements than those obtained in previous studies. Employing two different sets of stellar parameters, we find that this star has "normal" $\alpha$-element abundances for its metallicity. We otherwise derive abundances that are in good agreement with the majority of results reported in the literature.

$$
\text { 3.2.5. } B D+24^{\circ} 1676
$$

We note that there is some ambiguity about the evolutionary state of $\mathrm{BD}+24^{\circ} 1676$. Kinman (1999) draws attention to this star as a potentially interesting subdwarf with an 


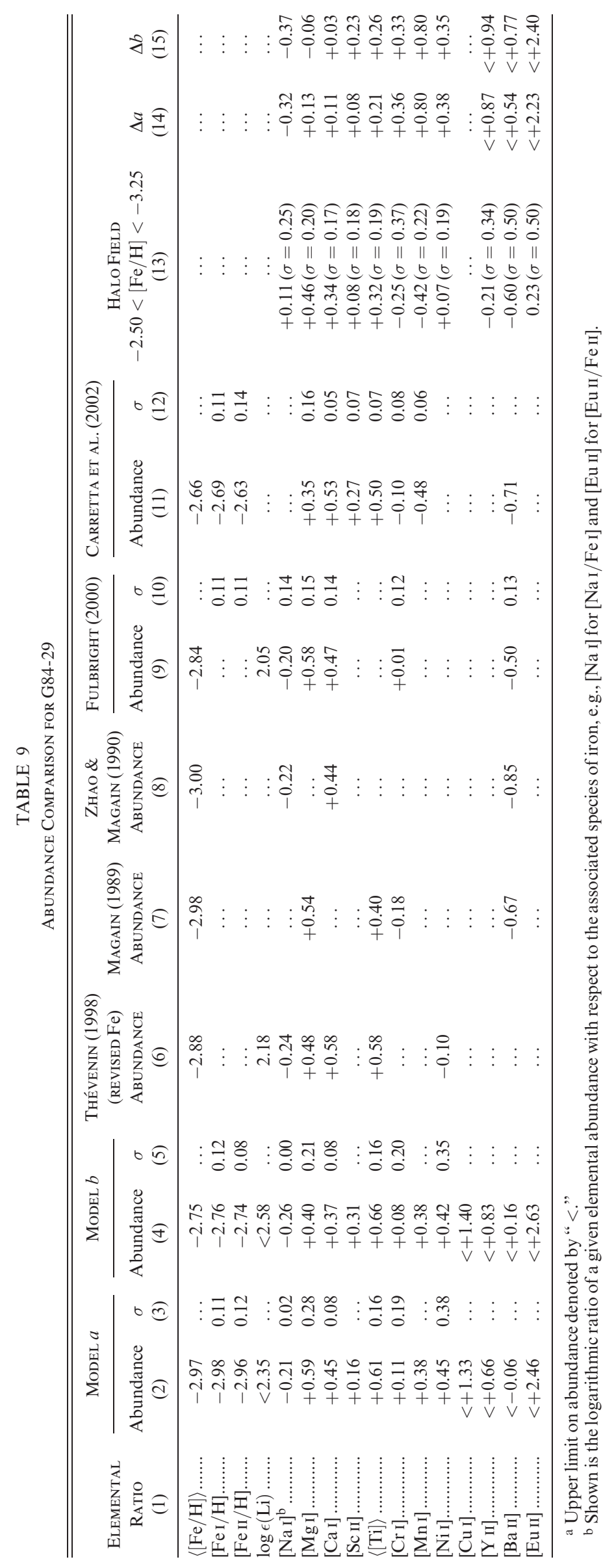


inferred $\left|Z_{\max }\right| \geq 10 \mathrm{kpc}$. However, the stellar parameters employed in our analysis $\left[\left(T_{\text {eff }}, \log g\right)=(6250 \mathrm{~K}, 3.45)\right]$ correspond to a more evolved star. Our $\log g$ value does satisfy the ionization equilibrium constraint and is also in accord with the $\log g$ calculated using equation (1) and the Hipparcos parallax, $3.79_{-1.22}^{+0.49}$. We also note that the spectroscopic constraints for this star can also be satisfied by a cooler model $\left[\left(T_{\text {eff }}, \log g\right)=(6050 \mathrm{~K}, 3.00)\right]$. However, these stellar parameters would then place the star on a color-magnitude diagram in a position far from any reasonable choice of stellar evolutionary isochrone (e.g., Bergbusch \& VandenBerg 1992; Straniero, Chieffi, \& Limongi 1997; or the updated Revised Yale Isochrones by Yi et al. 2001).

We present a catalog of abundances for this star in Table 10. Results for $\mathrm{BD}+24^{\circ} 1676$ were included in the "abundances from high-resolution spectra of kinematically interesting halo stars" by Stephens \& Boesgaard (2002). Our abundances, to within the errors, are in accord. Although we derive an $\mathrm{Ni}$ abundance that is elevated with respect to the usual trend, there is a large associated error $([\mathrm{Ni} / \mathrm{Fe}]=0.30 ; \sigma=0.19)$. The bulk of the results obtained in the present analysis are in accord with previously reported results by both James (2000) and Stephens \& Boesgaard (2002).

\subsection{Evidence of Binarity in $G 84-29$ and $B D+24^{\circ} 1676$ ?}

In Figure 7 we present the radial velocity information for stars G84-29 and BD $+24^{\circ} 1676$. The data from Latham et al. (2002) for both of these stars are plotted with the individual measurement error estimates taken from their Table 2, overplotted with a dashed line indicating the mean heliocentric radial velocity and dotted lines indicating the $1 \sigma$ range in the internal error estimates from their Table 3. Neither star shows variations from the mean that exceed the expected statistical variations based on the error estimates of the radial velocity measurements. Close companions are ruled out for both stars.

In the case of G84-29, the previous literature shows a range in adopted stellar parameters that may be a function of the wavelength employed in the determination. Based on uvby photometry, Schuster \& Nissen (1989) derive a metallicity of $[\mathrm{Fe} / \mathrm{H}] \simeq-1.7$; based on (largely redder) highresolution, low-S/N spectroscopy, Carney et al. (1994) estimate a metallicity of $[\mathrm{Fe} / \mathrm{H}] \simeq-2.78$. The derived spectroscopic gravity is also inconsistent with the evolutionary state of the star as deduced from Hipparcos parallax data. Applying spectroscopic constraints of ionization equilibrium, Fuhrmann (1998) derived a $\log g$ of 3.5 and $[\mathrm{Fe} / \mathrm{H}]=-2.7$ for $T_{\text {eff }}=6330 \mathrm{~K}$. Employing equation (1) and using the Hipparcos parallax of $\pi=7.80 \pm 2.09$ mas, we derive a trigonometric gravity of $4.17_{-0.31}^{+0.16}$ for this temperature, which, even in the errors, is 1 dex higher than the value we derived imposing the ionization equilibrium constraint. While it is possible that the error in the Hipparcos parallax measurement suggests that the derived $\log g$ should not be relied upon, perhaps the poor agreement in parameters is the result of distortion by an otherwise unseen companion.

TABLE 10

AbUndance COMParison for BD $+24^{\circ} 1676$

\begin{tabular}{|c|c|c|c|c|c|c|c|c|}
\hline Elemental Ratio & \multicolumn{2}{|c|}{ THIS PAPER } & \multicolumn{2}{|c|}{ JAMES (2000) } & \multicolumn{2}{|c|}{ STEPHENS \& BoEsGaARD (2002) } & $\begin{array}{c}\text { HALo FIELD } \\
-2.25<[\mathrm{Fe} / \mathrm{H}]<-2.75\end{array}$ & $\triangle$ ABUNDANCI \\
\hline$\langle[\mathrm{Fe} / \mathrm{H}]\rangle \ldots \ldots \ldots \ldots \ldots$ & -2.49 & $\ldots$ & -2.52 & & -2.55 & 0.06 & $\ldots$ & $\ldots$ \\
\hline$[\mathrm{Fe}$ II $/ \mathrm{H}] \ldots \ldots \ldots \ldots \ldots \ldots$ & -2.48 & 0.15 & $\ldots$ & 0.04 & $\ldots$ & $\ldots$ & $\ldots$ & $\ldots$ \\
\hline $\log \epsilon(\mathrm{Li}) \ldots \ldots \ldots \ldots \ldots \ldots$ & 2.45 & $\ldots$ & 2.19 & $\ldots$ & $\ldots$ & $\ldots$ & $\ldots$ & $\ldots$ \\
\hline 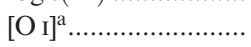 & +0.87 & 0.16 & +0.81 & 0.17 & $\ldots$ & $\ldots$ & $\ldots$ & $\ldots$ \\
\hline$\left[\mathrm{Al} \mathrm{I}^{\mathrm{b}}\right]^{\mathrm{b}} \ldots \ldots \ldots \ldots \ldots \ldots \ldots \ldots \ldots \ldots \ldots$ & $<+1.08$ & $\ldots$ & $\ldots$ & $\ldots$ & $\ldots$ & $\ldots$ & $-0.44(\sigma=0.40)$ & $<+1.52$ \\
\hline 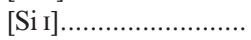 & $<+0.78$ & $\ldots$ & $\ldots$ & $\ldots$ & $\ldots$ & $\ldots$ & $+0.40(\sigma=0.24)$ & $<+0.38$ \\
\hline$[\mathrm{K}$ I $] \ldots \ldots \ldots \ldots \ldots \ldots \ldots \ldots \ldots \ldots \ldots \ldots \ldots \ldots \ldots$ & $<+0.87$ & $\ldots$ & $\ldots$ & $\ldots$ & $\ldots$ & $\ldots$ & $\ldots$ & $\ldots$ \\
\hline$[\mathrm{Ca} \mathrm{I}] \ldots \ldots \ldots \ldots \ldots \ldots$ & +0.43 & 0.09 & +0.45 & 0.04 & +0.37 & 0.09 & $+0.33(\sigma=0.14)$ & +0.10 \\
\hline$[\mathrm{Sc} \mathrm{II}] \ldots \ldots \ldots \ldots \ldots \ldots \ldots \ldots \ldots \ldots \ldots \ldots$ & +0.37 & 0.14 & $\ldots$ & $\ldots$ & $\ldots$ & $\ldots$ & $+0.09(\sigma=0.17)$ & +0.28 \\
\hline$\langle[\mathrm{Ti} / \mathrm{Fe}]\rangle \ldots \ldots \ldots \ldots \ldots$ & +0.40 & $\ldots$ & +0.56 & $\ldots$ & +0.46 & 0.09 & $\ldots$ & $\ldots$ \\
\hline$[\mathrm{Mn}$ I $] \ldots \ldots \ldots \ldots \ldots \ldots$ & +0.41 & $\ldots$ & $\ldots$ & $\ldots$ & $\ldots$ & $\ldots$ & $-0.31(\sigma=0.19)$ & +0.72 \\
\hline$[\mathrm{Ni}$ I $] \ldots \ldots \ldots \ldots \ldots \ldots$ & +0.30 & 0.19 & $\ldots$ & $\ldots$ & +0.10 & 0.17 & $+0.04(\sigma=0.16)$ & +0.26 \\
\hline$[\mathrm{Cu}$ I $] \ldots \ldots \ldots \ldots \ldots \ldots$ & +0.61 & $\ldots$ & $\ldots$ & $\ldots$ & $\ldots$ & $\ldots$ & $\ldots$ & $\ldots$ \\
\hline$[\mathrm{Y}$ II $] \ldots \ldots \ldots \ldots \ldots \ldots \ldots$ & +0.17 & $\cdots$ & $\ldots$ & $\cdots$ & $\ldots$ & $\cdots$ & $-0.24(\sigma=0.21)$ & +0.41 \\
\hline$[\mathrm{Ba}$ II] $\ldots \ldots \ldots \ldots \ldots \ldots \ldots \ldots \ldots \ldots \ldots \ldots \ldots$ & -0.49 & 0.04 & -0.40 & 0.06 & -0.42 & 0.15 & $-0.33(\sigma=0.52)$ & -0.16 \\
\hline$[$ La II $] \ldots \ldots \ldots \ldots \ldots \ldots$ & $<+2.14$ & $\ldots$ & $\ldots$ & $\ldots$ & $\ldots$ & $\ldots$ & $\ldots$ & $\ldots$ \\
\hline 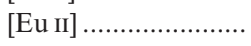 & $<+2.21$ & $\ldots$ & $\ldots$ & $\ldots$ & $\ldots$ & $\ldots$ & $\ldots$ & $\ldots$ \\
\hline
\end{tabular}

a $\left[\mathrm{O}_{\mathrm{I}}\right]$ denotes $[\mathrm{O} \mathrm{I} / \mathrm{Fe} \mathrm{II}]$. The remaining elements show the logarithmic ratio of a given elemental abundance with respect to the associated species of iron, e.g., [Na I] for [ $\mathrm{Na} / \mathrm{Fe} \mathrm{I}]$ and $[\mathrm{Eu} \mathrm{II}]$ for $[\mathrm{Eu} \mathrm{II} / \mathrm{Fe} \mathrm{II}]$.

b Upper limit on abundance denoted by " $<$." 


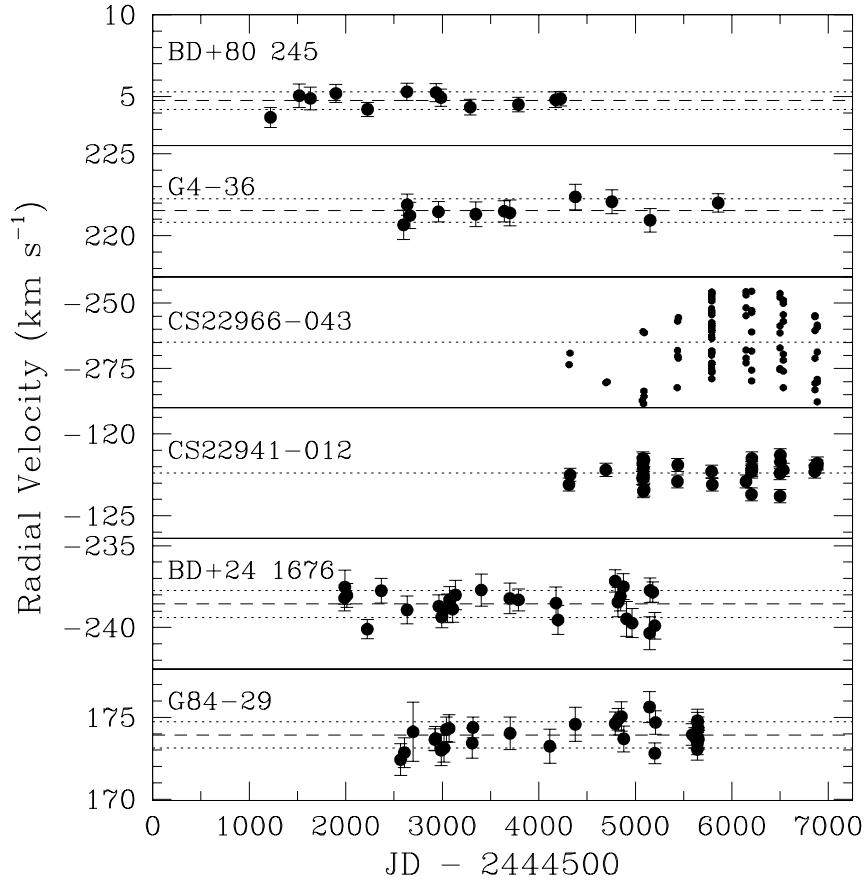

FIG. 7.- Radial velocity measurements and individual measurement error estimates taken from Latham et al. (2002) for BD $+80^{\circ} 245$, G4-36, G84-29, and BD $+24^{\circ} 1676$ and from Preston \& Sneden (2000) for CS 22966-043 and CS 22941-012. All but the panel for CS 22966-043 are on the same scale. Plotted in each panel is a dashed line at the value of the mean radial velocity. Dotted lines in the panels of the non-BMP stars indicate the $1 \sigma$ range in the estimate of the internal errors.

\subsection{Comparisons with Other Halo Stars of Similar Metallicity}

Many of the over- and underabundances derived in this study are easily seen in direct spectral comparisons. As noted in $\S 2$ and displayed in Figures 1-4, we obtained spectra of CS 22966-043 and G4-36 along with two field stars of comparable stellar parameters observed with the same instrumental configurations. All of the spectral comparisons clearly show $\alpha$-element and neutron-capture process element deficiencies in the low- $\alpha$ stars, as well as some of the overabundances we have derived in the Fe peak elements for G4-36 and CS 22966-043. An illustration of the unusually low $\mathrm{Ca}$ and $\mathrm{Ti}$ abundances in $\mathrm{BD}+80^{\circ} 245$ can be found in Figure 8 of Carney et al. (1997) in a spectrum overplot against G90-3 (an object of similar stellar parameters).

Figures 8-12 illustrate some of the abundance results for our metal-poor low- $\alpha$ stars in the context of other abundance studies of halo stars of comparable metallicities. For most of the elements presented in these figures, families of elements are grouped together. The symbols in each subpanel distinguish the results by research group. Some of the scatter in the abundance ratios can be attributed to systematic differences in the analyses performed by different groups. For instance, in the $[\mathrm{Na} / \mathrm{Fe}]$ subpanel of Figure 8, the values derived by Magain (1989) show little star-to-star variation, whereas these abundances are seen to be consistently higher than those obtained in more recent studies. For some elements, however, the scatter is astrophysical in origin. For instance, included in this and the following figures are the $[(\mathrm{Na}, \alpha) / \mathrm{Fe}]$ ratios from Fulbright $(2000,2002)$ and Stephens \& Boesgaard (2002), who found, in their respective studies, that stars with high total space velocities

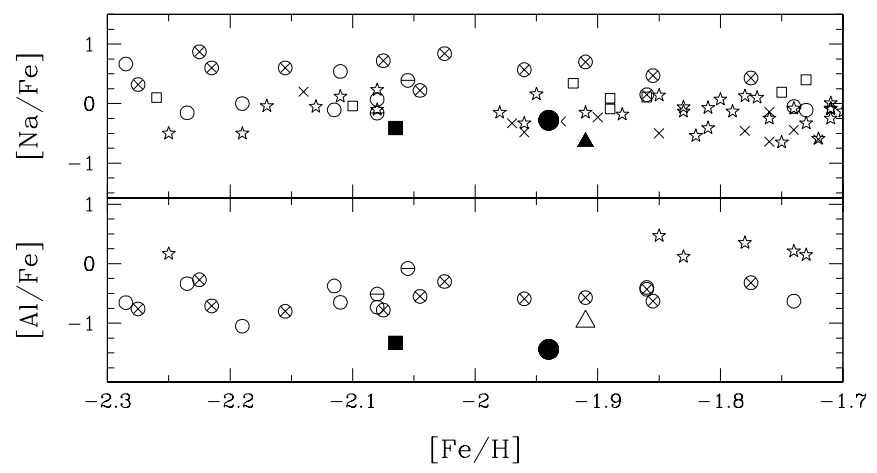

FIG. 8. - Abundances of light odd- $Z$ elements $[\mathrm{Na} / \mathrm{Fe}]$ and $[\mathrm{Al} / \mathrm{Fe}]$ as a function of $[\mathrm{Fe} / \mathrm{H}]$ for $\mathrm{BD}+80^{\circ} 245$ (filled squares), G4-36 (filled circles), and CS 22966-043 (filled triangles). Strict upper limits on derived abundances of our program stars for particular elements are denoted by hollow symbols. Small symbols denote results from other halo star studies: circled crosses for Magain (1989) and/or Zhao \& Magain (1990); circles with horizontal line for McWilliam et al. (1995a); open squares for Carretta et al. (2000); stars for Fulbright (2000); open circles for Johnson (2002); crosses for Stephens \& Boesgaard (2002). [See the electronic edition of the Journal for a color version of this figure.]

( $>300 \mathrm{~km} \mathrm{~s}^{-1}$ ) or stars with orbits with large $R_{\text {apo }}(>16 \mathrm{kpc})$ possess lower than average abundances of those elements. We find that the $[\mathrm{Na} / \mathrm{Fe}]$ ratios derived for our program stars are consistent with the low ratios found for the high-velocity/large- $R_{\text {apo }}$ stars.

In the case of Al, this study along with those of Magain (1989), McWilliam et al. (1995a, 1995b), and Johnson (2002) all employed the $\mathrm{Al}$ resonance line to derive the $\mathrm{Al}$ abundance, whereas the study by Fulbright (2000) employed the red $\mathrm{Al}$ lines. We refer the reader to a discussion by Ryan et al. (1996) that nicely summarizes the literature regarding the derived offsets in abundances from the red and violet $\mathrm{Al}$ features. For the metallicity range in which we are interested, the non-LTE calculations of Baumüller \& Gehren (1997) show that the difference in abundance from the violet features is $\sim 0.6$ dex lower than that derived from the red features. This value agrees well with the differences seen in the $[\mathrm{Al} / \mathrm{Fe}]$ subpanel of Figure 8, where the higher $[\mathrm{Al} / \mathrm{Fe}]$ ratio in the relatively more metal-rich stars was derived in a study employing the red features. However, even taking the non-LTE effects into account, the $[\mathrm{Al} / \mathrm{Fe}]$ ratios derived for our program stars are significantly lower than those found in other studies of comparable $[\mathrm{Fe} / \mathrm{H}]$.

In Figure 9 we show our $\alpha$-element abundance ratios for $[\mathrm{Mg} / \mathrm{Fe}],[\mathrm{Si} / \mathrm{Fe}]$, and $[\mathrm{Ca} / \mathrm{Fe}]$, in the context of those of other stars of comparable metallicities. All three even- $Z$ elements are significantly underabundant in our stars. Some systematic differences between the other studies are apparent. For instance, the $[\mathrm{Mg} / \mathrm{Fe}]$ ratios derived by Johnson (2002) are higher than average, whereas those of Mishenina \& Kovtyukh (2001) are, for the most part, lower than average. Our aim here is not to place all abundance studies on the same "system" - a worthwhile task but one that is beyond the scope of this paper. The main point here is that regardless of which system of analyses is chosen, the $\alpha$-element abundances of our low- $\alpha$ stars are significantly lower than those found in any of the other halo stars of comparable metallicities.

Figure 10 illustrates the abundance ratios we derived for the light $(A<26)$ iron peak elements, $[\mathrm{Sc} / \mathrm{Fe}],[\mathrm{Ti} / \mathrm{Fe}]$, 


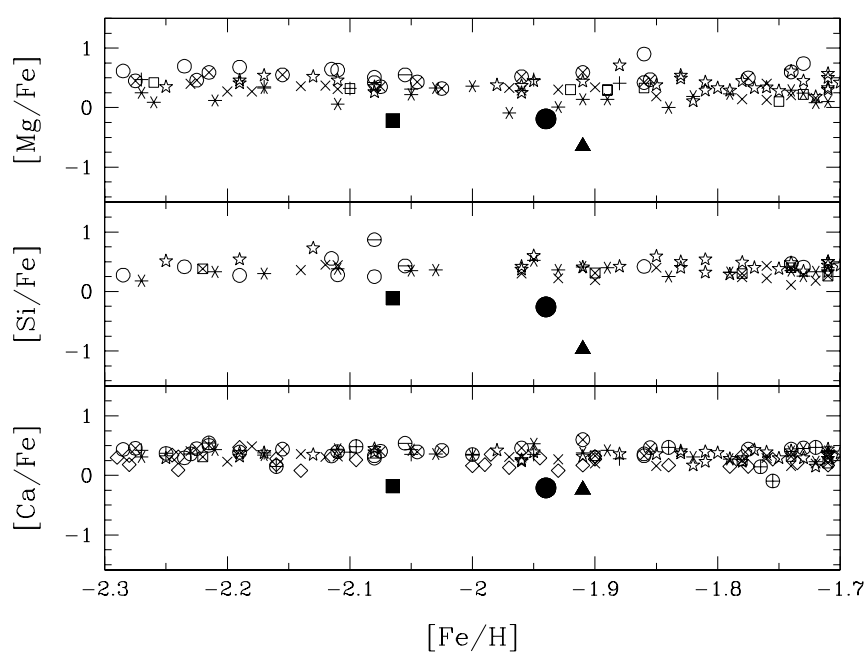

FIG. 9.-Abundances of $\alpha$-elements [Mg/Fe], [Si/Fe], and $[\mathrm{Ca} / \mathrm{Fe}]$ as a function of $[\mathrm{Fe} / \mathrm{H}]$ for $\mathrm{BD}+80^{\circ} 245$ (filled squares), G4-36 (filled circles), and CS 22966-043 (filled triangles). Small symbols denote results from other halo star studies: circled plus signs for Gilroy et al. (1988); circled crosses for Magain (1989) and/or Zhao \& Magain (1990); squared crosses for Gratton \& Sneden (1991) and/or Gratton \& Sneden (1994); plus signs for Nissen et al. (1994); circles with horizontal line for McWilliam et al. (1995a); open diamonds for Burris et al. (2000); open squares for Carretta et al. (2000); stars for Fulbright (2000); asterisks for Mishenina \& Kovtyukh (2001) and/or Mishenina et al. (2002); open circles for Johnson (2002); crosses for Stephens \& Boesgaard (2002). [See the electronic edition of the Journal for a color version of this figure.]

$[\mathrm{Cr} / \mathrm{Fe}]$, and $[\mathrm{Mn} / \mathrm{Fe}]$ in the context of other studies. As one marches through the subpanels describing the trends of the different elements, one can clearly see the shift in the relative abundance ratios of G4-36 and CS 22966-043 with respect to the mean ratios of stars of comparable metallicities. A similar but less pronounced shift may also be taking place in the abundance ratios of $\mathrm{BD}+80^{\circ} 245$.

Like Timmes, Woosley, \& Weaver (1995), in this figure we group titanium among the iron peak nuclei. However, as Timmes et al. (1995) note, it has been long known that $[\mathrm{Ti} / \mathrm{Fe}]$ ratios in metal-poor stars are enhanced over the scaled solar value (Wallerstein 1962) and that $[\mathrm{Ti} / \mathrm{Fe}]$ ratios instead follow the overabundances seen in $[\alpha / \mathrm{Fe}]$ ratios. In the case of CS 22966-043, when viewed against the general halo population, the abundance ratio we derive for $[\mathrm{Ti} / \mathrm{Fe}]$ appears to be enhanced, similar to the rest of the elemental abundance enhancements observed in this star near the $\mathrm{Fe}$ peak. However, when compared to the abundances derived for the other hot BMP star, CS 22941-012, the relative [Ti/Fe] ratio in CS 22966-043 appears to be "normal." Thus, the apparently high ratio of $[\mathrm{Ti} / \mathrm{Fe}]$ in CS 22966-043, when compared against the halo means, may simply be due to systematics in our abundance analysis of the BMP stars. However, a normal $[\mathrm{Ti} / \mathrm{Fe}]$ ratio in a star where both $\alpha$-element and Fe peak abundances are found to be unusual seems very unlikely. A possible explanation may be due to Ti being both an iron peak and $\alpha$-element. The "normal BMP" ratio of the [Ti/Fe] abundance in CS 22966-043 may be a result of the combined effects of Fe peak nucleosynthesis (present in CS 22966-043 material in abnormally high amounts) and the unusually low $\alpha$-element abundances, forcing the total $[\mathrm{Ti} / \mathrm{Fe}]$ to produce a normal BMP value.

Moving on to the abundance ratios of the heavier iron peak elements $(A>26),[\mathrm{Co} / \mathrm{Fe}],[\mathrm{Ni} / \mathrm{Fe}],[\mathrm{Cu} / \mathrm{Fe}]$, and

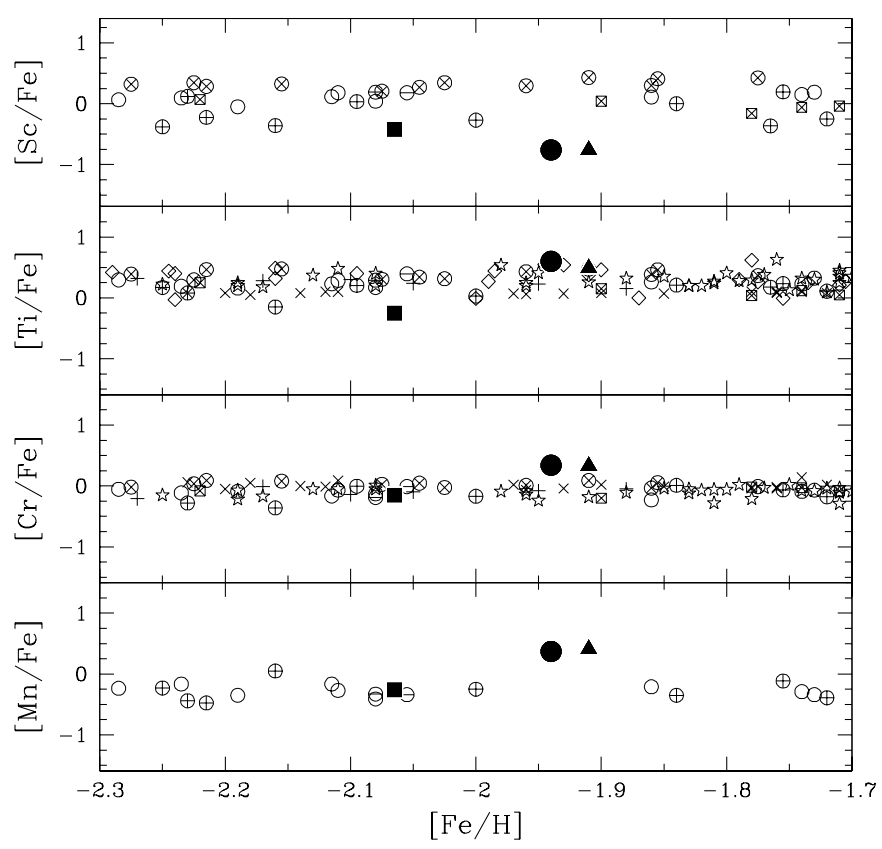

Fig. 10.-Abundance ratios of the light $(A<26) \mathrm{Fe}$ peak elements $[\mathrm{Sc} / \mathrm{Fe}],[\mathrm{Ti} / \mathrm{Fe}],[\mathrm{Cr} / \mathrm{Fe}]$, and $[\mathrm{Mn} / \mathrm{Fe}]$ as a function of $[\mathrm{Fe} / \mathrm{H}]$ for $\mathrm{BD}$ $+80^{\circ} 245$ (filled squares), G4-36 (filled circles), and CS 22966-043 (filled triangles). Small symbols denote results from other halo star studies: circled plus signs for Gilroy et al. (1988); circled crosses for Magain (1989) and/or Zhao \& Magain (1990); squared crosses for Gratton \& Sneden (1991) and/ or Gratton \& Sneden (1994); plus signs for Nissen et al. (1994); circles with horizontal line for McWilliam et al. (1995a); open diamonds for Burris et al. (2000); stars for Fulbright (2000); open circles for Johnson (2002); crosses for Stephens \& Boesgaard (2002). [See the electronic edition of the Journal for a color version of this figure.]

[Zn/Fe], Figure 11 shows that although the $[(\mathrm{Ni}, \mathrm{Zn}) / \mathrm{Fe}]$ ratios in G4-36 and CS 22966-043 are significantly overabundant with respect to halo stars of comparable metallicities, the upper limit for the abundance ratio of $[\mathrm{Cu} / \mathrm{Fe}]$ in G4-36 is in accord with the halo mean (we were unable to determine a useful upper limit in CS 22966-043). The dramatic difference in the $[(\mathrm{Cu}, \mathrm{Zn}) / \mathrm{Fe}]$ abundance ratios of this unusual star requires separate nucleosynthetic origins for these elements. This finding is in accord with results from investigations of the general metal-poor halo field star population (Sneden \& Crocker 1988; Sneden, Gratton, \& Crocker 1991a; Mishenina et al. 2002).

In Figure 12 our low- $\alpha$ stars also exhibit underabundances of neutron-capture elements when compared against other stars of comparable metallicities. Especially striking are the results for $\mathrm{BD}+80^{\circ} 245$, the star for which we have the highest $\mathrm{S} / \mathrm{N}$ and largest wavelength coverage.

The top panel of Figure 13 compares the results we obtained for the low- $\alpha$ stars against the average abundances found in the halo for stars of comparable metallicities (where the references for the halo star abundances are given in the captions of Figs. 8-12). The bottom panel of Figure 13 employs the difference of abundances for BMP star CS 22941-012 as a comparison for CS 22966-043 (instead of the halo mean). The differences presented in Figure 13 and Table 11 are in the sense of $\Delta[\mathrm{el} / \mathrm{Fe}]=$ this study - halo field or, where applicable, $\Delta[\mathrm{el} / \mathrm{Fe}]=\mathrm{CS} 22966-043-$ CS 22941-012. As shown in Table 11, abundances we derived for CS 22941-012 for elements other than Ti are in accord with those found in halo field stars of comparable 


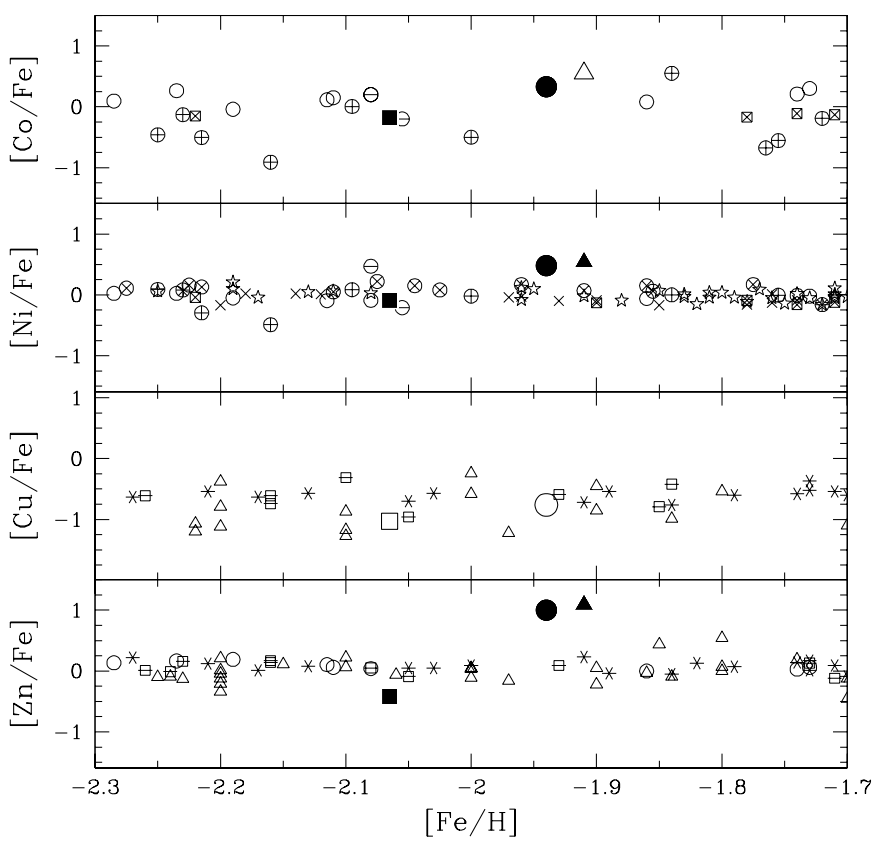

Fig. 11.-Abundances of the heavy $(A>26)$ Fe peak elements $[\mathrm{Co} / \mathrm{Fe}]$, $[\mathrm{Ni} / \mathrm{Fe}],[\mathrm{Cu} / \mathrm{Fe}]$, and $[\mathrm{Zn} / \mathrm{Fe}]$ as a function of $[\mathrm{Fe} / \mathrm{H}]$ for $\mathrm{BD}+80^{\circ} 245$ (filled squares), G4-36 (filled circles), and CS 22966-043 (filled triangles). Strict upper limits on derived abundances of our program stars for specific elements are denoted by hollow symbols. Small symbols denote results from other halo star studies: circled plus signs for Gilroy et al. (1988); circled crosses for Magain (1989) and/or Zhao \& Magain (1990); squared crosses for Gratton \& Sneden (1991) and/or Gratton \& Sneden (1994); squares with horizontal line for Sneden et al. (1991a); circles with horizontal line for McWilliam et al. (1995a); stars for Fulbright (2000); asterisks for Mishenina \& Kovtyukh (2001) and/or Mishenina et al. (2002); open triangles for F. Primas (2002, private communication; revised from Primas et al. 2000); open circles for Johnson (2002); crosses for Stephens \& Boesgaard (2002). [See the electronic edition of the Journal for a color version of this figure.]

metallicities. In the case of $\mathrm{Co}$, we only derived upper limits for the abundance of this element in both BMP stars; no $\Delta[\mathrm{Co} / \mathrm{Fe}]$ value for CS 22966-043 is plotted in Figure $13 b$.

Tables 9 and 10 contain similar comparisons for the abundances derived for stars G84-29 and BD +24 1676 against the average abundances found in lower metallicity halo field stars. In our analysis of G84-29, we derive the very high value of 0.45 for $[\mathrm{Ni} / \mathrm{Fe}]$. Combined with claims of low- $\alpha$ abundances in the literature, at first glance this star would appear to be an earlier generation low- $\alpha$ representative. However, further investigation shows that it is not likely: we are unable to confirm the low $\mathrm{Mg}$ result, and our Ni abundance has a very large associated error $(\sigma=0.38)$. Most of the remaining abundances in column (12) of Table 9 , are within $1 \sigma$ of the mean abundance of halo stars at comparable metallicities $(-2.75<[\mathrm{Fe} / \mathrm{H}]<-3.25)$; all but the differences in $\mathrm{Mn}, \mathrm{Ni}$, and $\mathrm{Y}$ fall within $2 \sigma$.

Like G84-29, most of the abundance differences in BD $+24^{\circ} 1676$, when compared with halo stars of similar metallicities (in this case, $-2.25<[\mathrm{Fe} / \mathrm{H}]<-2.75$ ), are within $1-$ $2 \sigma$ of the halo mean. The outstanding exception is $\mathrm{Mn}$, an elemental abundance ratio that also stands out in G84-29. Additional high-S/N data $(\sim 100)$ in the 3800-5000 $\AA$ wavelength range (in order to study more features) are required to resolve the degree of abundance anomalies in these two lower metallicity stars. As part of a related program, we

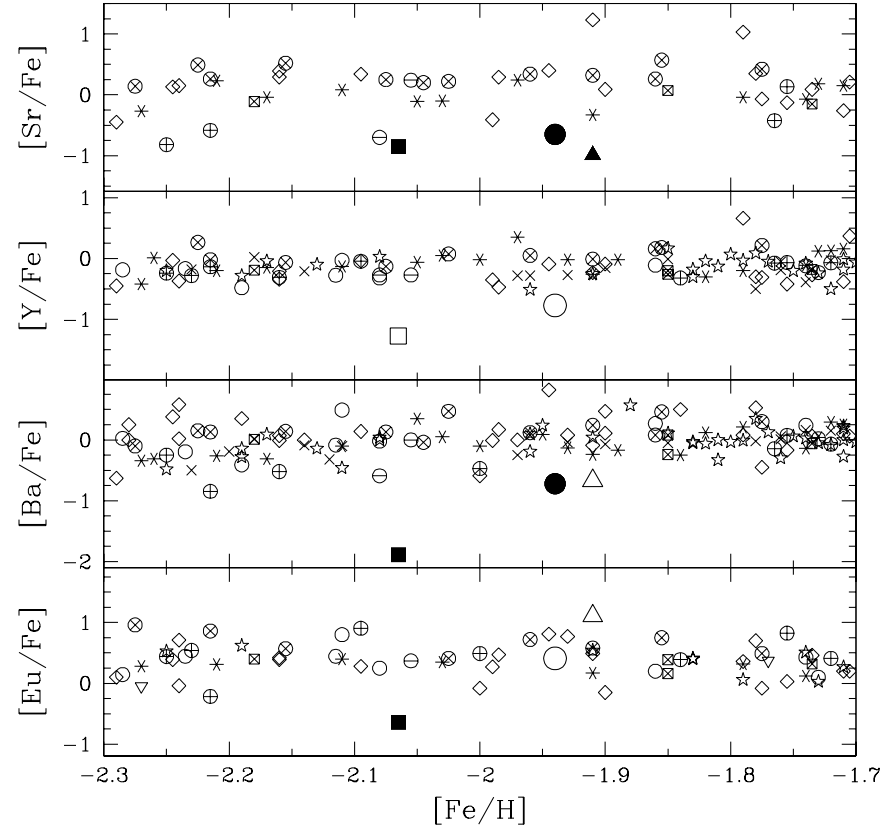

FIG. 12.-Abundances of the neutron-capture process elements $[\mathrm{Sr} / \mathrm{Fe}]$, $[\mathrm{Y} / \mathrm{Fe}],[\mathrm{Ba} / \mathrm{Fe}]$, and $[\mathrm{Eu} / \mathrm{Fe}]$ as a function of $[\mathrm{Fe} / \mathrm{H}]$ for $\mathrm{BD}+80^{\circ} 245$ (filled squares), G4-36 (filled circles), and CS 22966-043 (filled triangles). Strict upper limits on derived abundances of our program stars for specific elements are denoted by hollow symbols. Small symbols denote results from other halo star studies: circled plus signs for Gilroy et al. (1988); circled crosses for Magain (1989) and/or Zhao \& Magain (1990); downward-pointing triangles for Francois, Spite, \& Spite (1993); squared crosses for Gratton \& Sneden (1994); circles with horizontal line for McWilliam et al. (1995a); asterisks for Mishenina \& Kovtyukh (2001) and/or Mishenina et al. (2002); open diamonds for Burris et al. (2000); stars for Fulbright (2000); open circles for Johnson (2002); crosses for Stephens \& Boesgaard (2002). [See the electronic edition of the Journal for a color version of this figure.]

have acquired the necessary data, and the results will be presented in a future paper. We postpone further discussion of the results for these two low-metallicity stars to $\S$ 4.3.1.

\section{DISCUSSION}

The abundances derived in our three low- $\alpha$ stars are not shared by the typical metal-poor halo field star. Thus, they possess a chemical history different from those of the majority of the halo population. In this section we discuss some of the possible nucleosynthetic origins of the abundance anomalies observed in our stars.

\subsection{Supernova Nucleosynthesis: Comparisons to Model Yields}

The most obvious contribution from the yields of SNe Ia to Galactic evolution is the enrichment of the total iron content. This is most easily observed in the trend of a lowering of the $[\alpha / \mathrm{Fe}]$ ratio at about $[\mathrm{Fe} / \mathrm{H}] \simeq-1$ (Tinsley 1979; see, e.g., Pagel \& Tautvaisiene 1995, their Fig. 3; McWilliam 1997, his Fig. 1). Until now, however, we have not had a way in which to determine at what stage the SN Ia contributions possibly began.

In this study we attempt to match the abundances of our unusual low- $\alpha$ stars to various yields from supernova models in the literature. The yields we employed are those from 


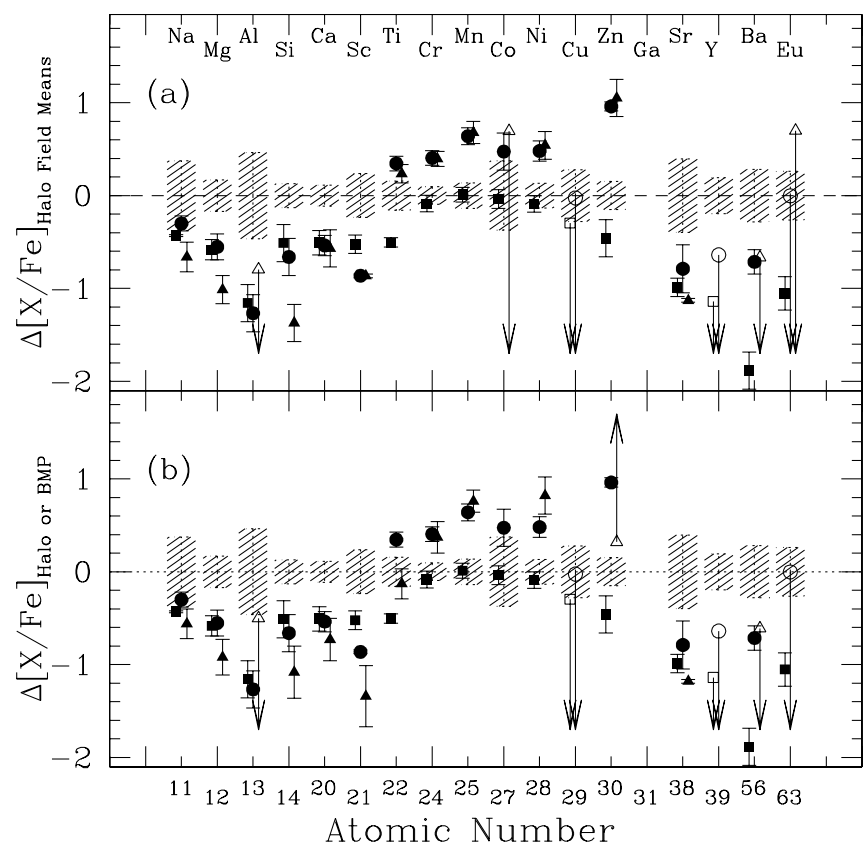

FIG. 13.-Abundance differences from Table 11 and $\sigma$ values from Table 5 grouped by atomic number. Abscissa shifts and symbols are those denoted in Fig. 5. Arrows indicate strict lower or upper limits on the differences; otherwise, shaded regions extending $1 \sigma$ from the mean are shown. Mean halo abundances in both panels are centered on $\Delta[\mathrm{X} / \mathrm{Fe}]=0$. (a) Offsets in the sense of $\Delta[\mathrm{X} / \mathrm{Fe}]_{\text {halo }}=$ this study - halo field means. (b) Offsets for CS 22966-043 with respect to BMP star CS 22941-012 and the combined errors of the abundances of both stars. [See the electronic edition of the Journal for a color version of this figure.] models representing SNe II from massive stars in the range of $10-50 M_{\odot}$ and those of SNe Ia resulting from thermonuclear explosions of electron-degenerate cores (Hoyle \& Fowler 1960), such as those found in massive white dwarfs in close binary systems. We did not include the calculated yields from SN Ia models of single-star progenitors of intermediate mass (see, e.g., carbon detonation and deflagration models by Arnett 1969; Fujimoto, Nomoto, \& Sugimoto 1976; Nomoto, Sugimoto, \& Neo 1976) or models of O-Ne$\mathrm{Mg}$ core collapse (see, e.g., Wanajo et al. 2003 and references therein).

Making the assumption that the total abundance of some element (X) we observe in these stars is simply explained as the result of a mix of SN Ia and SN II contributions, we can write the following ratio:

$$
\mathscr{R}_{\mathrm{obs}} \equiv \frac{M_{*}(\mathrm{X})}{M_{*}(\mathrm{Fe})}=\frac{N_{\mathrm{Ia}} M_{\mathrm{Ia}}(\mathrm{X})+N_{\mathrm{II}} M_{\mathrm{II}}(\mathrm{X})}{N_{\mathrm{Ia}} M_{\mathrm{Ia}}(\mathrm{Fe})+N_{\mathrm{II}} M_{\mathrm{II}}(\mathrm{Fe})},
$$

from which we can derive

$$
\frac{N_{\mathrm{Ia}}}{N_{\mathrm{II}}}=\frac{M_{\mathrm{II}}(\mathrm{X})-\mathscr{R}_{\mathrm{obs}} M_{\mathrm{II}}(\mathrm{Fe})}{\mathscr{R}_{\mathrm{obs}} M_{\mathrm{Ia}}(\mathrm{Fe})-M_{\mathrm{Ia}}(\mathrm{X})},
$$

where $\mathscr{R}_{\text {obs }} \equiv M_{*}(\mathrm{X}) / M_{*}(\mathrm{Fe})$ denotes the ratio of the mass of element $\mathrm{X}$ to the mass of iron in our star, $M_{\mathrm{Ia}}(\mathrm{X})$ and $M_{\mathrm{II}}(\mathrm{X})$ denote the mass of element $\mathrm{X}$ ejected from $\mathrm{SNe}$ Ia and SNe II, and $N_{\text {Ia }} / N_{\text {II }}$ represents the ratio of the number of SN Ia to SN II events that fit the observations and the synthesized mass of elements (X) and iron from the model yields.

TABLE 11

\begin{tabular}{|c|c|c|c|c|c|c|}
\hline Elemental Ratio & $\begin{array}{c}\text { Halo Field } \\
-1.75<[\mathrm{Fe} / \mathrm{H}]<-2.25\end{array}$ & $\Delta \mathrm{BD}+80^{\circ} 245$ & $\Delta \mathrm{G} 4-36$ & $\Delta \mathrm{CS} 22966-043$ & $\begin{array}{c}\text { CS 22941-012 } \\
{[\mathrm{el} / \mathrm{Fe}]}\end{array}$ & $\Delta$ CS 22966-043 \\
\hline$[\mathrm{Na} \mathrm{I}] \ldots \ldots \ldots \ldots \ldots \ldots$ & $+0.03(\sigma=0.38)$ & -0.44 & -0.31 & -0.67 & $-0.08(\sigma=0.03)$ & -0.56 \\
\hline$[\mathrm{Mg}$ I] $\ldots \ldots \ldots \ldots \ldots \ldots$ & $+0.37(\sigma=0.13)$ & -0.59 & -0.56 & -1.02 & $+0.27(\sigma=0.12)$ & -0.92 \\
\hline$[\mathrm{Al}$ I $] \ldots \ldots \ldots \ldots \ldots \ldots$ & $-0.10(\sigma=0.45)$ & -1.23 & -1.34 & $<-0.86^{\mathrm{a}}$ & $-0.47(\sigma=0.11)$ & $<-0.49$ \\
\hline$[\mathrm{Si}$ I] $\ldots \ldots \ldots \ldots \ldots \ldots \ldots \ldots \ldots \ldots \ldots \ldots$ & $+0.42(\sigma=0.15)$ & -0.53 & -0.68 & -1.39 & $+0.11(\sigma=0.20)$ & -1.08 \\
\hline$[\mathrm{Ca} \mathrm{I}] \ldots \ldots \ldots \ldots \ldots \ldots \ldots \ldots \ldots$ & $+0.31(\sigma=0.13)$ & -0.49 & -0.52 & -0.55 & $+0.49(\sigma=0.11)$ & -0.73 \\
\hline$[\mathrm{Sc}$ II] $\ldots \ldots \ldots \ldots \ldots \ldots \ldots \ldots \ldots \ldots$ & $+0.11(\sigma=0.26)$ & -0.53 & -0.87 & -0.87 & $+0.58(\sigma=0.33)$ & -1.34 \\
\hline$\langle[\mathrm{Ti}]\rangle \ldots \ldots \ldots \ldots \ldots \ldots$ & $+0.26(\sigma=0.16)$ & -0.55 & +0.28 & +0.34 & $+0.62(\sigma=0.16)$ & -0.02 \\
\hline$[\mathrm{Ti}$ I $] \ldots \ldots \ldots \ldots \ldots \ldots$ & $+0.26(\sigma=0.16)$ & -0.60 & +0.22 & +0.45 & $+0.68(\sigma=0.16)$ & +0.03 \\
\hline$[\mathrm{Ti}$ II] $\ldots \ldots \ldots \ldots \ldots \ldots \ldots$ & $+0.26(\sigma=0.16)$ & -0.51 & +0.34 & +0.23 & $+0.56(\sigma=0.13)$ & -0.07 \\
\hline$\left[\mathrm{V}_{\mathrm{I}}\right] \ldots \ldots \ldots \ldots \ldots \ldots \ldots \ldots \ldots \ldots$ & $-0.06(\sigma=0.23)$ & -0.33 & +0.38 & $\ldots$ & $\ldots$ & $\ldots$ \\
\hline$[\mathrm{Cr}$ I $] \ldots \ldots \ldots \ldots \ldots \ldots \ldots \ldots \ldots$ & $-0.06(\sigma=0.10)$ & -0.18 & +0.40 & +0.39 & $+0.04(\sigma=0.15)$ & +0.29 \\
\hline$[\mathrm{Cr}$ II $] \ldots \ldots \ldots \ldots \ldots \ldots \ldots \ldots \ldots \ldots$ & $-0.06(\sigma=0.10)$ & +0.21 & +0.54 & +0.42 & $+0.48(\sigma=0.16)$ & -0.12 \\
\hline$[\mathrm{Mn} \mathrm{I}] \ldots \ldots \ldots \ldots \ldots \ldots$ & $-0.28(\sigma=0.16)$ & +0.02 & +0.65 & +0.69 & $-0.36(\sigma=0.17)$ & +0.77 \\
\hline 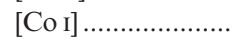 & $-0.27(\sigma=0.39)$ & -0.13 & +0.60 & $<-0.28$ & $<+0.29$ & $\ldots$ \\
\hline$[\mathrm{Ni}$ I $] \ldots \ldots \ldots \ldots \ldots \ldots$ & $+0.00(\sigma=0.14)$ & -0.09 & +0.36 & +0.54 & $+0.28(\sigma=0.13)$ & +0.26 \\
\hline$[\mathrm{Cu}$ I] $\ldots \ldots \ldots \ldots \ldots \ldots$ & $-0.63(\sigma=0.15)$ & $<-0.40$ & $<-0.13$ & $<+2.12$ & $<+1.28$ & $\ldots$ \\
\hline$[\mathrm{Zn}$ I] $\ldots \ldots \ldots \ldots \ldots \ldots$ & $+0.08(\sigma=0.08)$ & -0.50 & +0.92 & +1.01 & $<+0.77$ & $>+0.32$ \\
\hline$[\mathrm{Ga} I]^{\mathrm{b}} \ldots \ldots \ldots \ldots \ldots \ldots$ & $\ldots$ & $\ldots$ & $\ldots$ & $\ldots$ & $-0.87(\sigma=0.50)$ & +2.62 \\
\hline 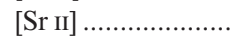 & $+0.17(\sigma=0.43)$ & -1.02 & -0.82 & -1.16 & $0.19(\sigma=0.01)$ & -1.18 \\
\hline$[\mathrm{Y}$ II $] \ldots \ldots \ldots \ldots \ldots \ldots$ & $-0.13(\sigma=0.20)$ & $<-1.14$ & $<-0.64$ & $<-0.38$ & $+0.55(\sigma=0.20)$ & $<-0.04$ \\
\hline$[\mathrm{Ba} I \mathrm{II}] \ldots \ldots \ldots \ldots \ldots \ldots$ & $-0.01(\sigma=0.32)$ & -1.88 & -0.70 & $<-0.66$ & $+0.06(\sigma=0.18)$ & $<-0.73$ \\
\hline$[\mathrm{La}$ II $] \ldots \ldots \ldots \ldots \ldots \ldots$ & $+0.07(\sigma=0.30)$ & -0.89 & $<1.31$ & $\ldots$ & $\ldots$ & $\ldots$ \\
\hline$[\mathrm{Eu} \mathrm{II}] \ldots \ldots \ldots \ldots \ldots \ldots$ & $+0.41(\sigma=0.26)$ & -1.05 & $<+0.01$ & $\ldots$ & $\ldots$ & $\ldots$ \\
\hline
\end{tabular}

Abundances in Our Low- $\alpha$ Stars Compared with Halo Field Stars

a Upper limit on abundance or difference denoted by " <"; lower limit on abundance difference denoted by " $>$."

b No Ga abundances for metal-poor stars exist in the literature. 


\subsubsection{Accreting White Dwarf Models of Iwamoto et al. (1999)}

We used the compilation of nucleosynthesis products of SN II and SN Ia models generated by Iwamoto et al. (1999, their Table 3) in which they report the yields from various models and tabulate the full decay of all unstable species. The SN II yields in the table are from calculations by Nomoto \& Hashimoto (1988), Hashimoto, Nomoto, \& Thielemann (1989), Hashimoto et al. (1996), and Thielemann, Nomoto, \& Hashimoto (1996) integrated over a Salpeter (1955) initial mass function (IMF) with SN II progenitor star masses from 10 to $50 M_{\odot}$. All of the SN Ia yields in the Iwamoto et al. (1999) table are modeled thermonuclear explosions of accreting white dwarfs. The table includes "W7," a Chandrasekhar mass " fast" deflagration model (Nomoto, Thielemann, \& Yokoi 1984; Thielemann, Nomoto, \& Yokai 1986; Hix \& Thielemann 1996), and "W70," the same hydrodynamical model as W7 but corresponding to zero initial metallicity. The "DD" models are all of a "slow" deflagration delayed detonation type. The "CDD" and "WDD" models differ from each other in the central ignition density and the "DD1," "DD2," and "DD3" models differ from each other in the density at the deflagration-detonation transition.

In our simple approach, we assume that the individual models could represent in some way the accumulated average and total output of supernova products prior to the birth of our stars. Equivalently, our stars could have been in close proximity to Type Ia explosions that deposited SN Ia residue on the surfaces of our stars. Whether formed from material enriched by early SN Ia events or merely witnesses, we assume that Type Ia material was deposited into preexisting SN II material. We also make two additional initial assumptions: no $s$-process contributions are necessary to explain the observed abundance ratios, and the contributed yields are fully represented by the models in Table 3 of Iwamoto et al. (1999).

We first tested the results of the model yields against the observed solar abundance ratios. Using the solar abundances of $\mathrm{O}, \mathrm{Na}, \mathrm{Mg}, \mathrm{Al}$, and $\mathrm{Si}$ from the compilation of Anders $\&$ Grevesse (1989) and excluding the results from the DD1 models (for which Iwamoto et al. 1999 also derived anomalous ratios), we derive a value of $\left\langle N_{\text {Ia }} / N_{\text {II }}\right\rangle_{\odot}=0.24 \pm 0.07$. This is in good agreement with literature values of Galactic ratios of SN Ia to SN II events: $0.12<\left(N_{\text {Ia }} / N_{\text {II }}\right)_{\odot}<0.36$ (van den Bergh \& Tammann 1991); 0.15 (Tsujimoto et al. 1995), based on similar nucleosynthetic arguments employing fits from 14 elements and their isotopes; and $0.187<\left(N_{\text {Ia }} / N_{\text {II }}\right)_{\odot}<0.257$ (Iwamoto et al. 1999), from the fit to the ratio of $[\mathrm{Si} / \mathrm{Fe}]$. with their DD2 SN Ia model.

We also performed trial determinations employing the suggested revision of the oxygen abundance from $\log \epsilon(\mathrm{O})=8.93$ (Anders \& Grevesse 1989) to 8.80 (Reetz 1999), 8.74 (Holweger 2001), and 8.69 (Allende Prieto, Lambert, \& Asplund 2001). These revisions resulted in $\left\langle N_{\text {Ia }} / N_{\text {II }}\right\rangle$ ratios of $0.28 \pm 0.07,0.29 \pm 0.09$, and $0.31 \pm 0.11$, respectively, with the $[\mathrm{O} / \mathrm{Fe}]$ ratio alone yielding ratios of $\left\langle N_{\mathrm{Ia}} / N_{\mathrm{II}}\right\rangle_{\mathrm{O}_{\text {new }}}=0.31,0.37$, and 0.43 , respectively. The result of using any of the newer $\mathrm{O}$ abundances produces a larger scatter in the ratios derived from the different elements. However, we have no way to determine whether the less than satisfactory results for the revised $\mathrm{O}$ abundance are due to errors in the input abundances or the model yields. We therefore tentatively dropped $[\mathrm{O} / \mathrm{Fe}]$ from our $\left\langle N_{\text {Ia }} / N_{\text {II }}\right\rangle$ determinations, recognizing that the evidence for this decision is not entirely compelling. Thus, employing the ratios of $[(\mathrm{Na}, \mathrm{Mg}, \mathrm{Al}, \mathrm{Si}) / \mathrm{Fe}]$. yields an $\mathrm{SN}$ Ia to $\mathrm{SN}$ II ratio of $\left\langle N_{\text {Ia }} / N_{\text {II }}\right\rangle_{\odot}=0.25 \pm 0.06$.

For other elements, the simple approach fails. When employing the abundances of N, Sc, Ti, V, Co, Ni, Cu, and $\mathrm{Zn}$, among others, we derive negative quantities in the SN ratios that are driven by the denominator of equation (3). Further discussion of the poor agreement for the prediction of the abundances of these elements by the SN models is beyond the scope of this paper, but clearly a better understanding is required as to why no combination of SN models is able to reproduce the abundances of these elements in the Sun (see, e.g., Timmes et al. 1995 and references therein).

As another sanity check, we also tested the results of the model yields against the observed abundances of CS 22892052, a star believed to have witnessed one (or only a few) previous SN event(s), of Type II only (Cowan et al. 1995, 1997; McWilliam et al. 1995a, 1995b; Sneden et al. 1994, 1996, 2000, 2002, 2003). The SN Ia to SN II ratio we derive from the published ratios of $[(\mathrm{Na}, \mathrm{Mg}, \mathrm{Si}) / \mathrm{Fe}]$ is $\left\langle N_{\text {Ia }} / N_{\text {II }}\right\rangle_{\text {CS 22892-052 }}=0.01 \pm 0.11$. Thus, we find that the formulation of equation (3) yields sensible results in this case: in the material out of which CS 22892-052 was born, contributions from $\mathrm{SNe}$ Ia had yet to arise.

We summarize the $\left(N_{\text {Ia }} / N_{\text {II }}\right)$ ratios we derive using the models of Iwamoto et al. (1999) in Table 12, along with the elemental ratios employed to derive those ratios. For our low- $\alpha$ star BD $+80^{\circ} 245$, of the elements that yield reasonable results in the Sun, there is a reasonable agreement in the $\alpha$-element abundances of $\mathrm{Mg}$ and $\mathrm{Si}$, as well as the light odd-element $\mathrm{Na}$ between the various models. The $\left\langle N_{\text {Ia }} / N_{\text {II }}\right\rangle$ ratio we derive for $\mathrm{BD}+80^{\circ} 245(0.58 \pm 0.21)$ is higher than that found in the Sun or averaged over the Galaxy but does correspond well to the idea that the low- $\alpha$ abundances in this star may be due to an iron enhancement resulting from $\mathrm{SNe}$ Ia. The results for other light elements, however, such as $\mathrm{Al}$, are not well matched to the results employing $\alpha$-elements. This suggests that there are contributors unaccounted for, or poorly accounted for, in either equation (2) or the model yields. While it appears, overall, that the abundances in this star are driven by SN Ia contributions, we assume that they were deposited into preexisting $\mathrm{SN}$ II material.

In the $\mathrm{SN}$ models we employed, the $\mathrm{Si}$ mass yield from $\mathrm{SNe}$ II is comparable to the yield from $\mathrm{SNe}$ Ia, whereas the yields of $\mathrm{Na}$ and $\mathrm{Al}$ are over 100 times greater in SNe II than $\mathrm{SNe}$ Ia. The abundances of isotopes with more neutrons than protons are expected to be sensitive to the neutron excess. Referring to their SN II models, Tsujimoto et al. (1995) note that some odd- $Z$ elements and neutron-rich species are produced in the deepest layers of the ejecta of SNe II and that the resulting abundances are "very sensitive to the neutron excess of the very thin layer, and are therefore subject to large uncertainties." Perhaps the relatively poor fit of the yields to the $\mathrm{Al}$ abundance in $\mathrm{BD}+80^{\circ} 245$ can be attributed to this particular uncertainty in the SN II yields.

In the case of CS 22966-043, we derive an even higher ratio of $\mathrm{SNe}$ Ia to $\mathrm{SNe}$ II. From the abundance ratios of $[(\mathrm{Na}, \mathrm{Mg}, \mathrm{Si}) / \mathrm{Fe}]$ we derive $\left\langle N_{\mathrm{Ia}} / N_{\mathrm{II}}\right\rangle=1.29 \pm 0.08$. As illustrated in Figure 5, the chemical abundances of CS 22966-043 closely resemble those derived for G4-36, and, as shown in Table 12, we derive similar $\left\langle N_{\text {Ia }} / N_{\text {II }}\right\rangle$ ratio results, as expected. In contrast to CS 22966-043, we would 
TABLE 12

SN Ratios Derived from Stellar Abundance Fits to SN Model Yields

\begin{tabular}{|c|c|c|c|}
\hline Star & $\left(N_{\text {Ia }} / N_{\text {II }}\right)$ & {$[\mathrm{el} / \mathrm{Fe}]$} & SN Ia Model Fit \\
\hline \multirow[t]{4}{*}{ Sun } & $0.24 \pm 0.07$ & $\mathrm{O}, \mathrm{Na}, \mathrm{Mg}, \mathrm{Al}, \mathrm{Si}$ & 1 \\
\hline & $0.25 \pm 0.06$ & $\mathrm{Na}, \mathrm{Mg}, \mathrm{Al}, \mathrm{Si}$ & 1 \\
\hline & $0.22 \pm 0.05$ & $\mathrm{Na}, \mathrm{Mg}, \mathrm{Si}$ & 1 \\
\hline & $0.18 \pm 0.01$ & $\mathrm{Na}, \mathrm{Mg}, \mathrm{Si}$ & 2 \\
\hline \multirow{2}{*}{ 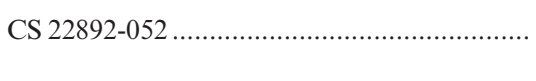 } & $0.01 \pm 0.11$ & $\mathrm{Na}, \mathrm{Mg}, \mathrm{Si}$ & 1 \\
\hline & $0.01 \pm 0.10$ & $\mathrm{Na}, \mathrm{Mg}, \mathrm{Si}$ & 2 \\
\hline \multirow{2}{*}{ 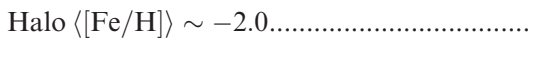 } & $0.09 \pm 0.11$ & $\mathrm{Na}, \mathrm{Mg}$ & 1 \\
\hline & $0.09 \pm 0.10$ & $\mathrm{Na}, \mathrm{Mg}$ & 2 \\
\hline \multirow[t]{2}{*}{ 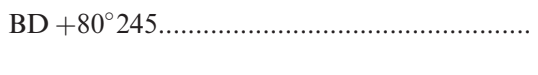 } & $0.58 \pm 0.21$ & $\mathrm{Na}, \mathrm{Mg}, \mathrm{Si}$ & 1 \\
\hline & $0.47 \pm 0.21$ & $\mathrm{Na}, \mathrm{Mg}, \mathrm{Si}$ & 2 \\
\hline \multirow[t]{4}{*}{ G4-36 } & $0.88 \pm 0.78$ & $\mathrm{Na}, \mathrm{Mg}, \mathrm{Si}$ & 1 \\
\hline & $1.79 \pm 0.13$ & $\mathrm{Si}$ & 1 \\
\hline & $0.43 \pm 0.10$ & $\mathrm{Na}, \mathrm{Mg}$ & 1 \\
\hline & $0.45 \pm 0.12$ & $\mathrm{Na}, \mathrm{Mg}, \mathrm{Si}$ & 2 \\
\hline \multirow[t]{2}{*}{ 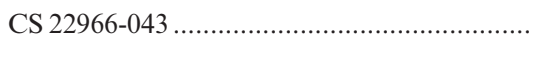 } & $1.29 \pm 0.08$ & $\mathrm{Na}, \mathrm{Mg}, \mathrm{Si}$ & 1 \\
\hline & $1.22 \pm 0.01$ & $\mathrm{Na}, \mathrm{Mg}, \mathrm{Si}$ & 2 \\
\hline \multirow[t]{2}{*}{ CS 22941-012.. } & $0.15 \pm 0.10$ & $\mathrm{Na}, \mathrm{Mg}, \mathrm{Si}$ & 1 \\
\hline & $0.13 \pm 0.09$ & $\mathrm{Na}, \mathrm{Mg}, \mathrm{Si}$ & 2 \\
\hline \multirow[t]{2}{*}{ 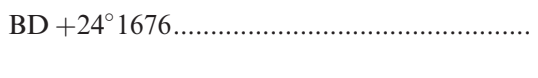 } & $0.17 \pm 0.19$ & $\mathrm{Na}, \mathrm{Mg}$ & 1 \\
\hline & $0.16 \pm 0.20$ & $\mathrm{Na}, \mathrm{Mg}$ & 2 \\
\hline \multirow[t]{2}{*}{ 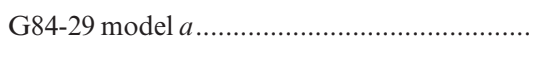 } & $0.16 \pm 0.21$ & $\mathrm{Na}, \mathrm{Mg}$ & 1 \\
\hline & $0.15 \pm 0.26$ & $\mathrm{Na}, \mathrm{Mg}$ & 2 \\
\hline \multirow[t]{2}{*}{ 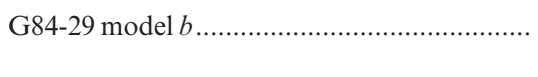 } & $0.21 \pm 0.22$ & $\mathrm{Na}, \mathrm{Mg}$ & 1 \\
\hline & $0.20 \pm 0.27$ & $\mathrm{Na}, \mathrm{Mg}$ & 2 \\
\hline \multirow{2}{*}{ 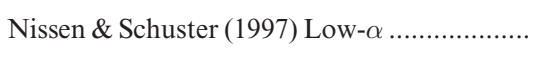 } & $0.23 \pm 0.11$ & $\mathrm{Na}, \mathrm{Mg}, \mathrm{Si}$ & 1 \\
\hline & $0.20 \pm 0.10$ & $\mathrm{Na}, \mathrm{Mg}, \mathrm{Si}$ & 2 \\
\hline \multirow[t]{2}{*}{ Nissen \& Schuster (1997) other halo ................. } & $0.08 \pm 0.03$ & $\mathrm{Na}, \mathrm{Mg}, \mathrm{Si}$ & 1 \\
\hline & $0.07 \pm 0.03$ & $\mathrm{Na}, \mathrm{Mg}, \mathrm{Si}$ & 2 \\
\hline \multirow[t]{2}{*}{ Pal $12 \ldots$} & $0.38 \pm 0.22$ & $\mathrm{Na}, \mathrm{Mg}, \mathrm{Si}$ & 1 \\
\hline & $0.29 \pm 0.18$ & $\mathrm{Na}, \mathrm{Mg}, \mathrm{Si}$ & 2 \\
\hline \multirow[t]{2}{*}{ Rup 106} & $0.39 \pm 0.32$ & $\mathrm{Na}, \mathrm{Mg}, \mathrm{Si}$ & 1 \\
\hline & $0.34 \pm 0.31$ & $\mathrm{Na}, \mathrm{Mg}, \mathrm{Si}$ & 2 \\
\hline \multirow[t]{2}{*}{ 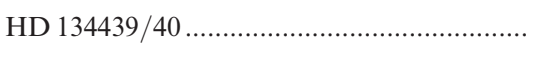 } & $0.41 \pm 0.18$ & $\mathrm{Na}, \mathrm{Mg}, \mathrm{Si}$ & 1 \\
\hline & $0.34 \pm 0.19$ & $\mathrm{Na}, \mathrm{Mg}, \mathrm{Si}$ & 2 \\
\hline
\end{tabular}

REFERENCES.- (1) Iwamoto et al. 1999. (2) Höflich et al. 1995; Höflich \& Khokhlov 1996; Höflich et al. 1998; Domínguez et al. 2001; Höflich et al. 2002.

expect our comparison BMP halo object, CS 22941-012, to show not only a relatively lower $\left\langle N_{\text {Ia }} / N_{\text {II }}\right\rangle$ ratio, but also one that would be lower than the solar $\left\langle N_{\text {Ia }} / N_{\text {II }}\right\rangle$ ratio, since more time has elapsed for the solar nebula to have incorporated additional SN Ia material relative to that of SNe II. This is indeed the case.

Employing the SN Ia model yields from Iwamoto et al. (1999), the results for G4-36 are rather ambiguous; there is poor agreement in the $\left\langle N_{\mathrm{Ia}} / N_{\mathrm{II}}\right\rangle$ ratios derived from the $[(\mathrm{Na}, \mathrm{Mg}, \mathrm{Si}) / \mathrm{Fe}]$ abundance ratios. However, if we treat the elements in two separate fits, one can group the ratios of $[(\mathrm{Na}, \mathrm{Mg}) / \mathrm{Fe}]$, which yields an $\mathrm{SN}$ Ia to SN II ratio of $\left\langle N_{\text {Ia }} / N_{\text {II }}\right\rangle=0.43 \pm 0.1$, and a separate fit from the ratio of [Si/Fe], which yields an $\mathrm{SN}$ Ia to $\mathrm{SN}$ II ratio of $\left\langle N_{\text {Ia }} / N_{\text {II }}\right\rangle=1.79 \pm 0.13$. Given that the abundance we derived for silicon is reliable (as previously discussed in $\S 3.2 .2$ ), the difference in the results could be due to a variety of reasons, among which include the following:

1. Perhaps SN II yields integrated over a Salpeter IMF are inappropriate, and perhaps some of the lower mass progenitors had not had sufficient time to contribute to the medium out of which G4-36 formed. However, by $[\mathrm{Fe} / \mathrm{H}]$ of -2 , if metallicity can be roughly tied to a "Galactic clock," based on lifetimes of low-mass SNe II $\sim 8-10 M_{\odot}$, all of the SN II masses should be represented.

2. Perhaps a significant contribution to the material in this star came from an SN II progenitor that had a mass cut different from the standard adopted by Iwamoto et al. (1999). A different mass cut could indeed produce a difference in the ratios of certain elements, $\mathrm{Si}$ among them, but this would then require G4-36 to have been made from material involving more than one odd SN event occurrence, not only the predominance of SNe Ia but a "special" SN II condition. We feel that this is unlikely to have taken place.

3. Perhaps the SN Ia yields that contributed to the mix out of which this star formed are not represented in the selection provided by Iwamoto et al. (1999).

In the next section we explore the dependence of the derived $\left\langle N_{\text {Ia }} / N_{\text {II }}\right\rangle$ ratio on the yields from alternative $\mathrm{SN}$ Ia scenarios.

\subsubsection{Additional and Alternative SN Ia Scenarios}

We expanded on the simple approach described in $\S 4.1 .1$ and incorporated a greater range of SN Ia model yields by including those produced by scenarios investigated in five additional studies: Höflich, Wheeler, \& Thielemann (1998), 
who employed a different modeling approach from the Iwamoto et al. (1999) group; Höflich \& Khokhlov (1996), who investigated $\mathrm{SNe}$ Ia resulting from merger scenarios; Höflich, Khokhlov, \& Wheeler (1995), who derived subChandrasekhar mass models; Domínguez, Höflich, \& Straniero (2001), who modeled a set of low-metallicity CO white dwarf yields; and Höflich et al. (2002), who investigated subluminous $\mathrm{SNe}$ Ia.

From the theoretical point of view, a wide variety of SN Ia scenarios are possible. Although nowadays we see that specific models (i.e., the explosion of Chandrasekhar mass models) dominate the sample, in the past other mechanisms may have dominated (e.g., mergers). The implications are systematic changes in the SN population. The identification of these changes is critical for the use of SNe Ia in cosmological applications, such as measuring the "dark energy"; that is, intrinsic SN effects must be separated from cosmological changes.

There are far more model yield predictions than there are observable elemental abundances that have trustworthy values. Thus, rather than sampling over all of the abundance yields currently available from a wide variety of SN scenarios, we selected a subset of 45 models that sampled well the " average " yield values of various progenitor scenarios.

The best-fit $\left\langle N_{\text {Ia }} / N_{\text {II }}\right\rangle$ ratio is defined here to be the result derived from the $[(\mathrm{Na}, \mathrm{Mg}, \mathrm{Si}) / \mathrm{Fe}]$ ratios that produced the least corresponding scatter. Table 12 contains our $\left\langle N_{\text {Ia }} / N_{\text {II }}\right\rangle$ results employing the models of both Iwamoto et al. (1999) and Höflich's group, along with the elemental abundance ratios employed.

As expected, the abundances for the $r$-process-rich star CS 22892-052 fit all of the SN Ia models. This very mild "test" of our methodology allows us to confirm our hypothesis but provides no additional constraints as to which SN Ia models produce reasonable chemical abundance yields. In the solar case, a handful of models reproduce the known abundance ratios equally well. ${ }^{13}$ The bestfit yields included those from a standard Chandrasekhar mass accreting white dwarf progenitor with "slow" deflagration detonation burning, a pulsating delayed detonation model of a "close to Chandrasekhar mass" white dwarf, and a few models of subsolar mass progenitors that explode by He detonation.

Approximately half the models we employed fit the abundance ratios of $\mathrm{BD}+80^{\circ} 245$, including numerous solar-metallicity $\mathrm{C} / \mathrm{O}$ white dwarf models from main-sequence progenitors of $\sim 5 M_{\odot}$ developed to investigate subluminous SNe Ia, such as SN 1999by. The abundances of G4-36, like those of $\mathrm{BD}+80^{\circ} 245$, are satisfied equally well by a number of different SN Ia yields. In the case of CS 22966043 , only a few models seem to fit the abundances we derived. In contrast, over half of the models we employed in these tests fit the abundances of the comparison BMP star, CS 22941-012.

We find that all of the stars listed in Table 1, as well as the extremely metal-poor CS 22892-052 and the Sun, are satisfied by yields produced in three different models, one of the close to Chandrasekhar mass pulsating delayed detonation types and two of the subsolar mass He detonation types. While higher $\mathrm{S} / \mathrm{N}$ data and improved atomic parameters

\footnotetext{
${ }^{13}$ The value derived using the Höflich group models is within the errors we derived for the result based on the Iwamoto et al. (1999) models, albeit barely.
}

will help shrink the error bars on the derived abundances, our ability to distinguish between models is mostly limited by the number of elements we have employed in the fits of the models to the derived abundances. To expand the present investigation in the most useful way, the existing $\mathrm{SN}$ models need to be refined. The SN model yields that provide a better match to the solar abundances will also likely provide better matches to the abundances of these much lower metallicity stars.

Worth pursuing would be an improved identification of the likely models that best represent the progenitors that contributed to the interstellar mix out of which our stars were born. Not only would the identification provide additional information regarding the nucleosynthetic histories of the low- $\alpha$ objects, among other stars, but, because the abundance pattern resulting from an SN characterizes the explosion physics, detailed abundance determinations would provide unique glimpses into the properties of the earliest SNe.

Recent work by Baumgartner, Mushotzky, \& Horner (2003) of X-ray elemental abundances in the intracluster medium employs yields from $\mathrm{SNe}$ in a way similar to that which we have derived in this study. Baumgartner et al. (2003) attempted to fit SN ratios to the intracluster abundances as a function of temperature (i.e., cluster mass). They find that the results based on the ratios of $[\mathrm{Si} / \mathrm{Fe}]$ and $[\mathrm{S} / \mathrm{Fe}]$ do not track each other as expected (they are both $\alpha$-elements) and suggest that either the elements are distributed and not homogenized in the intracluster gas or the $\mathrm{SN}$ models are in need of refinement. Although temperature inhomogeneities could play a role, given our results we suspect that the model yields need to be explored further.

In order to more accurately sum up the contributions from various nucleosynthesis sites in our stars in a more general form, equation (2) should be revised to include not only additional as yet unspecified sources but also novae and $\mathrm{AGB}$ yields [although $\sum N M(\mathrm{Fe})$ for novae and $\mathrm{AGB}$ are $\sim 0$, other elemental contributions are expected]. We also note the need to incorporate the history of SN yields. This last point is not realizable at this time. During early times in Galactic star formation, fractional yields from individual $\mathrm{SNe}$ have a larger effect, when local inhomogeneities would be expected to dominate the chemical mix out of which lowmetallicity stars formed. What is required is a set of models whose yields are based on the particular and sometimes inhomogeneous chemical abundances out of which individual SN progenitors were born.

\subsubsection{Results from $S N I b$ and High-Energy SN II ("Hypernova") Yields}

High-energy SNe.-Nucleosynthetic yields resulting from the core-collapse explosions of very massive Population III stars have been calculated by Umeda \& Nomoto (2002). Their explorations included studying the effects of variations in the ejecta resulting from the Si-burning regions on the abundances of $\mathrm{Zn}, \mathrm{Co}, \mathrm{Mn}, \mathrm{Cr}$, and $\mathrm{V}$ with respect to $\mathrm{Fe}$ with the aim of determining whether observed trends could be better explained with high-energy SN ("hypernova") models.

We employed the yields of the hypernova models in place of the SN II model calculations by Nomoto \& Hashimoto (1988), Hashimoto et al. $(1989,1996)$, and Thielemann et al. (1996) and rederived the $\left\langle N_{\text {Ia }} / N_{\text {II }}\right\rangle$ ratios for our program 
stars in order to ascertain whether the hypernovae might better reproduce their abundances. In no case, regardless of the mass and energy of the input model, does the variation between the abundances employed improve over those of standard SNe II. We find that the unusual abundances of our stellar trio cannot be explained by the hypernova models of Umeda \& Nomoto (2002).

Type Ib SNe.-Like SNe II, SNe Ib and SNe Ic are associated with young stellar populations (van Dyk 1992). Unlike SNe Ia, they are often radio sources (Weiler \& Sramek 1988). The radio emission suggests that the $\mathrm{SN}$ shock is plowing into a surrounding medium, consistent with the idea of mass loss from the progenitor star (see, e.g., review by Wheeler 2003 and references therein). $\mathrm{SNe} \mathrm{Ib}$ (and $\mathrm{SNe} \mathrm{Ic}$ ) are thought to be the result of massive stars stripped of their outer layers of hydrogen (and helium) through mass loss during their WolfRayet evolutionary phase. Thus, SNe Ib and SNe Ic basically differ from $\mathrm{SNe}$ II in their $\mathrm{H}$ and He layers but are otherwise expected to be similar in their cores.

Woosley, Langer, \& Weaver (1995) provide nucleosynthetic yields for models describing SNe Ib and SNe Ic for helium stars in the range of 4-20 $M_{\odot}$. For many elements, their models produce yields that span the range of yields produced by SN Ia and SN II models. For instance, the sodium yield from WLW20 is on the order of the sodium yield calculated by the Iwamoto SN II models. In contrast, the sodium yield from WLW4 is the same as that produced by the W7 SN Ia model, a full 2 orders of magnitude lower. We therefore attempted to fit their yields to our abundance ratios in two ways: (1) by treating the $\mathrm{SNe} \mathrm{Ib} / \mathrm{Ic}$ as alternatives to the SN II yields, and (2) by treating them as alternatives to the suite of SN Ia yields. In neither case were we able to find a satisfactory agreement among the abundances for any of the stars. Based on the yields of Woosley et al. (1995), we find that $\mathrm{SNe} \mathrm{Ib} / \mathrm{Ic}$ do not make a significantly unique contribution to the abundances in the stars studied here.

\subsection{Alternative Explanations?}

In this section we explore the possibility that the source of the abundance anomalies may have arisen from other sources: contamination from an $s$-process-enhanced partner, formation from AGB-enriched material, effects due to chemically stratified atmospheres, and atmospheric pollution from nearby SNe Ia.

\subsubsection{Binarity and Mass Transfer?}

Mass transfer from a binary partner is often invoked to explain the abundances observed in $\mathrm{Ba}$ II and $\mathrm{CH}$ stars, red giants that exhibit enhanced abundances of carbon and $s$ process elements (see, e.g., McClure \& Woodsworth 1990 and references therein). Since all $\mathrm{Ba}$ II and $\mathrm{CH}$ stars likely possess companions and the abundance anomalies in these stars are similar to those found in the classical (higher luminosity) carbon stars on the AGB, the peculiarities of the $\mathrm{Ba}$ II and $\mathrm{CH}$ stars have been explained by mass transfer from former AGB star companions overflowing into the Roche lobe of the observed star.

Lower luminosity stars near and above the main sequence possessing strong $\mathrm{CH}$ features and enhanced $s$-process elemental abundances were discovered by Bond (1974). These subgiant $\mathrm{CH}$ stars have also been found to be binaries (McClure 1997), and like their red giant counterparts, their abundance anomalies are the result of the atmospheric pollution from a former AGB star. Can the abundances of our low- $\alpha$ stars be explained by AGB pollution from a binary partner?

In Figure 7 we illustrate the available radial velocity measurements for our program stars. Data for the two BMP stars were taken from Preston \& Sneden (2000); data for the rest of our stars were taken from Latham et al. (2002). In the case of CS 22966-043, Preston \& Sneden (2000) derive a nearly circular orbit with a period of 317.0 days; using the same data, Carney et al. (2001) confirm the result, deriving a period of 306.4 days. G4-36 exhibits no variability; there is no evidence for a close binary with a period of order 10-20 yr. According to McClure \& Woodsworth (1990), periods longer than this would indicate systems sufficiently widely separated that mass transfer probably could not have taken place. The longest period subgiant CH star reported by McClure (1997) is 4144 days. Barring an unfavorable orbital inclination, G4-36 does not appear to have a period within this range. Ruling out AGB mass transfer for G4-36 would also rule it out as an explanation for CS 22966-043, which, although a known variable star in an SX Phe system, seems to share the abundance over- and underenhancements of G4-36. Finally, no radial velocity variability is observed in $\mathrm{BD}+80^{\circ} 245$, which exceeds the expected statistical variations.

\subsubsection{Formation from AGB-enriched Material?}

Some of the isotopes of elements that are usually associated with Fe peak material can originate in the $s$-process in AGB stars. Could CS 22966-043 and G4-36 (the two low- $\alpha$ stars that possess enhanced Fe peak abundances) have been formed from AGB-enriched material? In other words, could $s$-processing be responsible for the enhancements we observe in $\mathrm{Zn}$ and $\mathrm{Ga}$ without enhancing $\mathrm{Sr}$ or $\mathrm{Ba}$, for which we observe enormous underabundances in these stars with respect to the halo stars of comparable metallicities?

Raiteri, Gallino, \& Busso (1992, their Table 6) show, among other elements, the relative main and light $s$-process contributions to the solar abundances of Co, Ni (usually considered to be $\mathrm{Fe}$ peak elements), $\mathrm{Cu}, \mathrm{Zn}$ (whose origins in the solar system are still controversial), Ga, and Ge, as well as Sr and Y (usually considered to be light $s$-process elements). We employed the Raiteri et al. (1992) percentages to determine whether there exist statistically significant correlations with the corresponding abundances and upper limits in G4-36 and CS 22966-043. Where +1 or -1 would indicate a perfect correlation or anticorrelation, both Pearson's linear correlation coefficients $\left(-0.13>R^{2}>-0.51\right)$ and Spearman's rank correlation values $\left(+0.24>r_{s}>-0.48\right)$ are less than significant.

In our stars, the upper limit we derive for the ratio of $[\mathrm{Cu} / \mathrm{Fe}]$ is in accord with the abundances reported for other metal-poor stars. If anything, $\mathrm{Cu}$ is depleted, rather than enhanced (see Figs. 5 and 11). In both G4-36 and CS 22966043 , the abundance of $\mathrm{Zn}$, and probably $\mathrm{Ga}$, is enhanced. We sought but were unable to detect Ge, which might have provided additional clues about the origins of the $\mathrm{Zn}$ (and Ga) contributions.

Since none of the lightest $s$-process isotopes are found to be enhanced and no overabundance of the light $s$-process elements $\mathrm{Sr}$ or $\mathrm{Y}$ is detected, nor are any overabundances detected in the heavier neutron-capture elements of our stellar trio, there exists no evidence of pollution from an 
$s$-process site. Thus, we cannot explain the $\mathrm{Fe}$ peak abundance enhancements in this manner.

\subsubsection{Chemically Stratified Atmospheres?}

Gallium has not previously been reported in abundance studies of metal-poor halo stars. However, it is often observed and found to be enhanced in the class of objects known as chemically peculiar (CP) stars. Of present-day main-sequence stars of spectral types B8-F0, $20 \%-25 \%$ possess chemical peculiarities that vary systematically along the spectral type sequence (Preston 1974).

The peculiar abundances of the $\mathrm{CP}$ stars are attributed to diffusion in the atmospheres producing chemical stratification, a diffusion that is likely radiatively driven (see, e.g., Richer, Michaud, \& Turcotte 2000 and references therein). In order for chemical stratification due to selective radiation to work, the stellar envelope needs to be extremely quiet (Michaud 1970), a condition that can only be satisfied in stars undergoing no envelope convection. CS 22966-043 is an SX Phe pulsating star, exhibiting pulsations driven by convective instabilities, and thus cannot be a representative of an old population of CP stars. If not CP stars themselves, could the atmospheres of our objects have been polluted by binary partners or could our stars have formed from $\mathrm{CP}$-enriched material?

Takada-Hidai, Sadakane, \& Jugaku (1986) derived abundance enhancements of $\mathrm{Ga}$ of up to $\sim 3$ dex and found a strong correlation with $T_{\text {eff }}$ in a large sample of CP stars. In a subsequent study, Sadakane, Jugaku, \& Takada-Hidai (1988) found large over- and underabundances of $\mathrm{Zn}$. Although they found no correlation between $\mathrm{Zn}$ and $T_{\text {eff }}$ or with either of the neighboring elements, $\mathrm{Cu}$ and $\mathrm{Ga}$, they did find that both $\mathrm{Ga}$ and $\mathrm{Cu}$ follow the same $T_{\text {eff }}$ trends. In contrast to this, for G4-36 the abundances of $\mathrm{Zn}$ and Ga rise and fall together, and in none of our stars is $\mathrm{Cu}$ observed to be enhanced. Our sample of stars also do not match other abundance correlations seen in the CP group. For instance, the CP stars do not share the depletions of $\mathrm{Al}, \mathrm{Sr}$, or $\mathrm{Ba}$ that are observed in our stars. Instead, $\mathrm{Al}$ and the rare earth elements are among those elements found to be enhanced in CP stars.

Finally, in models where magnetically amplified radiative accelerations reproduce the $\mathrm{Al}, \mathrm{Ga}, \mathrm{Ni}$, and $\mathrm{Zn}$ enhancements observed in CP stars, the models also reproduce the accompanying $\mathrm{Mg}$ and $\mathrm{Ca}$ enhancements (see, e.g., Alecian $\&$ Stift 2002), in contrast to what is found in our stars, where the $\alpha$-element abundances are uniformly suppressed.

Given the severe physical limits within which the CP mechanism operates, along with the ability of the radiative diffusion models to successfully reproduce the abundance trends of the CP stars and the lack of correlation in those abundances with the differing elemental abundance trends observed in our stars, we cannot explain the abundances of our sample by invoking CP stars, either intrinsically, as atmospheric pollutants, or as chemically enriching the material out of which our stars were born.

\subsubsection{Extremely Metal-poor Stars Polluted by SNe Ia?}

In this section we consider the possibility that our stars were born from much less chemically enriched material and that the presence of SN Ia material does not come from early SN Ia events but was, instead, deposited by SN Ia events occurring at much later times.
Tsujimoto, Shigeyama, \& Yoshii (2002) investigated the chemical evolution of the light odd- $Z$ elements $\mathrm{Na}$ and $\mathrm{Al}$ with respect to the ratio of $[\mathrm{Mg} / \mathrm{H}]$ for very early generations of metal-poor stars formed from material produced in $\mathrm{SNe}$ II. Comparing our results to their Figure 1, the abundance ratios and upper limits we derive for $[\mathrm{Al} / \mathrm{Mg}],[\mathrm{Na} / \mathrm{Mg}]$, and $[\mathrm{Mg} / \mathrm{H}]$ in $\mathrm{BD}+80^{\circ} 245$, G4-36, and CS $22966-043$ would place all three stars in the same area of the diagram as those objects identified by Tsujimoto et al. (2002) as having been born in the first few generations of stars.

Turning next to the $[\mathrm{Ca} / \mathrm{Mg}]$ ratio, when viewed in the context of the McWilliam et al. (1995a) results for " 33 of the most metal-poor stars" then known, the ratios derived for our stars are in reasonable agreement with those derived by McWilliam et al. (1995a, see their Fig. 24). While the $[\mathrm{Ca} / \mathrm{Mg}]$ ratio we derive for CS $22966-043$ is very low, it is in accord with the three lowest $[\mathrm{Ca} / \mathrm{Mg}]$ ratios found by McWilliam et al. (1995a).

In the $[\mathrm{Ti} / \mathrm{Mg}]-[\mathrm{Mg} / \mathrm{H}]$ plane described by the McWilliam et al. (1995a) sample, the abundance we derive for CS 22966-043 would place it in an outlying position very close to that of the largest outlier in their Figure 25. The outlier, CS 22898-027, is also among their trio of low- $[\mathrm{Ca} / \mathrm{Mg}]$ stars. On the basis of these abundance similarities in the $\alpha$ element ratios, it is tempting to consider that CS 22966-043 and CS 22898-027 may share similar nucleosynthetic histories. However, the behavior of the neutron-capture elements in these two stars is vastly different: the Ba-to-iron ratio in CS 22898-027 is in excess of 2000 times the value derived for CS 22966-043. Other neutron-capture elements, including $\mathrm{La}$ and $\mathrm{Eu}$, are found to be similarly overabundant in CS 22898-027. The object is a CH subgiant (McWilliam et al. 1995a; Bartkevičius 1996) such as those discussed in $\S$ 4.2.1. The abundances of CS 22898-027 can thus be explained by the accretion of material from a former AGB star companion.

Since the abundance of neutron-capture material is low in our stars, we investigated the possibility that the abundances of elements such as $\mathrm{Sr}, \mathrm{La}$, and Ba could be attributed solely to $r$-process contributions. The rise in the Galactic abundances of $s$-process elements is not observed to take place until approximately $-3<[\mathrm{Fe} / \mathrm{H}]<-2$ (see, e.g., Spite \& Spite 1978, their Fig. 5; Burris et al. 2000, their Figs. 5,7 , and 9). Thus, if our stars began life as extremely metalpoor stars with iron enrichment taking place later, we would expect to find that elements with large $s$-process components are deficient with respect to those with large $r$-process components.

We compared the abundances derived for our low- $\alpha$ stars against theoretical predictions from Burris et al. (2000) for some of the elements that trace the relative contributions from $r$ - and $s$-processing. The $r$-process contribution of a given element is estimated by subtracting the calculated $s$ process yield from the observed solar abundance. In studies of the neutron-capture-rich star CS 22892-052, Sneden et al. (2003 and references therein) found a better match between their observed abundances and the scaled solar $r$ process determinations of Burris et al. (2000) than with those of Arlandini et al. (1999); in the case of the Cowan et al. (2002) study of $\mathrm{BD}+17^{\circ} 3248$, the opposite was found. We present here the results we obtained employing the Burris et al. (2000) $r$ - and $s$-process solar system abundance fractions but note that, for the elemental abundance ratios and associated errors we are concerned with here, the 
differences between the predictions of Arlandini et al. (1999) and Burris et al. (2000) are insignificant.

For our comparison, we employed the following elements (where the percentages listed are from Burris et al. 2000): Eu ( $97 \%$ of the solar abundance is formed via the $r$-process), $\mathrm{Sr}$ ( $89 \%$ of the solar abundance is formed via the $s$-process), $\mathrm{Ba}$ $(85 \% s)$, and $\mathrm{La}(75 \% s)$, along with $\mathrm{Zn}$ and $\mathrm{Ga}$, whose origins are less certain. We scaled the $r$-process curve from Burris et al. (2000) to fit the $\log \epsilon(\mathrm{Eu})$ value derived for $\mathrm{BD}$ $+80^{\circ} 245$ and then applied the same scaling for the Burris et al. (2000) $s$-process curve. Although our Ba abundance for $\mathrm{BD}+80^{\circ} 245$ falls below the total scaled solar $r+s$ value, the other neutron-capture element abundances ( $\mathrm{Sr}$ and $\mathrm{La}$ ) both fit the scaled $r+s$ curve very well. In fact, the same scaled solar $r+s$ curve fits the $\log \epsilon(\mathrm{Sr})$ abundance derived for all three stars. Both the $\log \epsilon(\mathrm{Ba})$ abundance for G4-36 and upper limit for CS 22966-043 also fit this curve. Therefore, employing the abundances we derived, we find that the low neutron-capture ratios with respect to iron are not due to contributions from $r$-process-only sites; the amount of neutron-capture material found in our stars requires a ratio of $s$ - to $r$-process contributions on the order of the ratio of $s: r$ contributions found in the Sun.

Thus, we cannot explain the abundances of our low- $\alpha$ stars as being a result of extremely low metallicity stars contaminated by material accreted from a nearby Type Ia explosion.

To sum up the results of our exploration of these alternative explanations, while it is possible that the atmospheres do not represent the interstellar material out of which they were born and instead exhibit the effects of pollution from another source, no object has been observed that displays abundances sufficiently similar to those we derive.

\subsection{Multiple Generations of Low- $\alpha$ Stars?}

4.3.1. Does an Earlier Generation Also Exist?

$\mathrm{BD}+80^{\circ} 245$, G4-36, and CS 22966-043 share a common low metallicity and low abundances of $\alpha$-elements and neutron-capture process elements. G4-36 and CS 22966-043 further share similar enhancements in their Fe peak abundances. Is there evidence that an earlier generation of stars possessing comparable abundance anomalies exists (or once existed)?

As discussed in $\S 2.4$, unusual abundances have been reported in previous studies of G4-89 and $\mathrm{BD}+24^{\circ} 1676$ that resemble those we derived for CS 22966-043 and G436. For $\mathrm{BD}+24^{\circ} 1676$ we find an SN Ia to SN II ratio (from $[(\mathrm{Na}, \mathrm{Mg}) / \mathrm{Fe}])$ of $\left\langle N_{\mathrm{Ia}} / N_{\mathrm{II}}\right\rangle_{\mathrm{BD}+24^{\circ} 1676}=0.17 \pm 0.2$, which is not significantly different from that which was derived for the BMP halo star CS 22941-012. For G84-29, for which we find normal halo star $\alpha$-element abundances, we derive $\left\langle N_{\mathrm{Ia}} / N_{\mathrm{II}}\right\rangle_{\mathrm{G} 84-29}=0.18 \pm 0.24$, which is neither significantly different from the rest of the halo nor the value one would expect with no SN Ia input.

We sought further evidence in the literature of low- $\alpha$ abundances for very low metallicity stars. Giridhar et al. (2001) report abundances of $\mathrm{Na}, \mathrm{Mg}, \mathrm{Al}, \mathrm{Si}, \mathrm{Ca}, \mathrm{Sc}$, and $\mathrm{Ti}$ in CS 22169-035 $([\mathrm{Fe} / \mathrm{H}]=-2.86)$ that are all $1<\sigma<3$ below the mean of the abundances derived for halo stars of comparable metallicity (as derived from the data presented in Tables 9 and 10). Thus, at first glance, the object seems to resemble BD $+80^{\circ} 245$. Employing the Giridhar et al. (2001) abundances, we attempted to derive an $\left\langle N_{\text {Ia }} / N_{\text {II }}\right\rangle$ ratio as we did for the other stars. With these abundances, however, the scatter in ratios derived for the various abundances is much larger and no SN Ia model stood out as being significantly better than the rest. Combined with the iron abundance Giridhar et al. (2001) derived, the $\mathrm{Al}$ and $\mathrm{Si}$ abundances yield negative ratios, $\mathrm{Mg}$ yields a ratio of $\left\langle N_{\text {Ia }} / N_{\text {II }}\right\rangle_{\text {CS 22169-035 }}=0.51 \pm 0.91$, and $\mathrm{Na}$ yields a ratio of $\left\langle N_{\text {Ia }} / N_{\text {II }}\right\rangle_{\text {CS } 22169-035}=2.72 \pm 1.62$. The abundances of this star are unusual, but it does not follow that they are a result of SN Ia contributions. We recommend that this star be subjected to further scrutiny. In particular, the abundances of the light $s$-elements of $\mathrm{Sr}$ and $\mathrm{Y}$, combined with abundances of $\mathrm{Cu}$ and $\mathrm{Zn}$, might shed further light on the nucleosynthetic history of this object.

Finally, we note that some extremely metal-poor stars also evidence low $\alpha$-element and low neutron-capture element abundances. For instance, two stars with $[\mathrm{Fe} / \mathrm{H}] \simeq-3.4$ found by McWilliam et al. (1995a) exhibit mildly low $\alpha$-element abundances along with either low $\mathrm{Sr}$ or low $\mathrm{Sr}$ and $\mathrm{Ba}$ ratios relative to iron. These abundances have been confirmed by Ryan et al. (1996), as well as in subsequent studies. McWilliam \& Searle (1999) suggest that the low neutron-capture abundances for these stars are what one might expect from a model of stochastic chemical evolution at extremely low metallicities. Thus, given the findings presented here, it would seem that lower metallicity analogs of our trio of low- $\alpha$ stars have not been uncovered.

\subsubsection{Comparisons with More Metal-rich, Low- $[\alpha / \mathrm{Fe}]$ Stars}

As shown in $\S 3.4$, our stars display unusual abundances in the context of other stars of comparably low metallicities. How do our low- $\alpha$ stars appear when viewed in the context of studies of more metal-rich but otherwise low- $\alpha$ stars?

In a detailed investigation of chemical abundance trends of halo and disk stars of metallicities in the range of $-1.3 \lesssim[\mathrm{Fe} / \mathrm{H}] \lesssim-0.5$, Nissen \& Schuster (1997) found that most of the halo stars in their sample possess significantly lower $\alpha$-element abundances than those of the disk stars with overlapping metallicities. The low- $\alpha$ stars they found also display underabundances in the ratios of $[\mathrm{Na} / \mathrm{Fe}]$, as well as small deficiencies in $[\mathrm{Ni} / \mathrm{Fe}]$. The abundance ratio trends observed in the Nissen \& Schuster (1997) stars resemble those we find in BD $+80^{\circ} 245$, albeit in a milder form.

When we apply the techniques of $\S 4.2$ and seek to match the abundances with various combinations of SN model yields, we derive $0.20 \pm 0.10<\left(N_{\text {Ia }} / N_{\text {II }}\right)_{\mathrm{NS} 97}<0.23 \pm 0.11$ for their low- $\alpha$ stars, a mean value comparable to that which we derive for the Sun. This high $\left\langle N_{\text {Ia }} / N_{\text {II }}\right\rangle$ ratio is consistent with adding more iron to the compositions of the low- $\alpha$ stars (from the yields of SN Ia events) than is required to explain the abundances of normal halo stars of comparable metallicities. This explanation is in accord with the idea that Nissen \& Schuster (1997) proposed: the stars should be viewed as being "iron-rich" as opposed to $\alpha$ poor; i.e., the low- $\alpha$ stars have a high iron content relative to a given $\alpha$-element content. In their corresponding scenario, Nissen \& Schuster (1997) propose that the low- $\alpha$ stars can thus be explained as being born out of gas that had been enriched by SN Ia material at an "unusually low metallicity." However, we note that no significant difference is seen in the $s$-process abundance ratios for $[(\mathrm{Y}, \mathrm{Ba}) /$ $\mathrm{Fe}$ ] between the halo and disk stars in the Nissen \& 
Schuster (1997) sample. Neither Y nor Ba is expected to emerge from SN Ia sites. If iron enrichment drove preexisting $\alpha$-element abundances in the halo stars to lower $[\alpha / \mathrm{Fe}]$ ratios, then one would expect that the iron enrichment should likewise have lowered any preexisting [s-process/ $\mathrm{Fe}]$ ratios by similar amounts. Further investigation of the origins of the Nissen \& Schuster (1997) sample of low- $\alpha$ stars would benefit from the knowledge of additional elements, such as those largely attributed to the $r$-process (e.g., $\mathrm{Eu})$.

With low- $\alpha$ abundances similar to those found in the Nissen \& Schuster (1997) study, stars in the outer halo globular cluster Pal $12\left([\mathrm{Fe} / \mathrm{H}] \sim-1 ; R_{\text {apo }}=15.9 \mathrm{kpc}, 2003\right.$ February revision of Harris 1996) also exhibit low values in the ratio $[\mathrm{Ni} / \mathrm{Fe}]=-0.34 \pm 0.14$ (Brown et al. 1997). However, for the abundances of elements largely created in the $s$ process, Brown et al. (1997) derive $[\mathrm{Ba} / \mathrm{Fe}]=0.13 \pm 0.14$ for the two stars in their sample, a value that is supported by their derivation $[\mathrm{La} / \mathrm{Fe}]=0.31 \pm 0.10$ and is unlike the low neutron-capture element abundances of either the Nissen \& Schuster (1997) stars or those of the present study. Finding that Pal 12 stars possess higher abundances of $s$-process material than the Nissen \& Schuster (1997) field stars may simply be the result of atmospheric contamination: it is possible that the observed cluster stars accreted material from earlier generations of AGB stars, which have long since shed their outer layers, during passages through the ISM of the cluster. However, not all clusters possess similar abundances of neutron-capture material with respect to iron. Employing abundance ratios of $[(\mathrm{Na}, \mathrm{Mg}, \mathrm{Si}) / \mathrm{Fe}]$, which excludes the information regarding the differences in the neutron-capture elemental abundances, we derive $0.29 \pm 0.18<\left(N_{\text {Ia }} / N_{\text {II }}\right)_{\text {Pal } 12}<0.38 \pm 0.22$.

Along with Pal 12, Brown et al. (1997) also studied a pair of stars in the lower metallicity outer halo cluster Rup 106 $\left([\mathrm{Fe} / \mathrm{H}]=-1.36 \pm 0.15 ; R_{\text {apo }}=18.5 \mathrm{kpc}, 2003\right.$ February revision of Harris 1996). Both Pal 12 and Rup 106 are approximately 3-4 Gyr younger than the average halo population (Pal 12, Gratton \& Ortolani 1988; Rup 106, Buonanno et al. 1990). Brown et al. (1997) found that the Rup 106 stars possess both lower $[\alpha / \mathrm{Fe}]$ ratios and a lower ratio of $[\mathrm{Ni} / \mathrm{Fe}](-0.46 \pm 0.08)$, and they also exhibit diminished abundance ratios of $s$-process elements with $[\mathrm{Ba} / \mathrm{Fe}]=-0.41 \pm 0.05$, which is supported by the upper limit found for La. Employing the $[(\mathrm{Na}, \mathrm{Mg}, \mathrm{Si}) / \mathrm{Fe}]$ ratios from Brown et al. (1997), we derive $0.34 \pm 0.32<$ $\left(N_{\text {Ia }} / N_{\text {II }}\right)_{\text {Rup 106 }}<0.39 \pm 0.32$. While the mean is essentially the same as that for Pal 12 , the error bars indicate that a more extensive analysis of Rup 106 abundances is required.

Consistent with the ratios derived in Pal 12 and Rup 106, King (1997) finds diminished $\alpha$-element abundances in the outer halo $\left(R_{\text {apo }}>40 \mathrm{kpc}\right.$; Carney et al. 1994) common proper-motion pair, HD 134439 and HD 134440 $([\mathrm{Fe} / \mathrm{H}] \sim-1.50)$, which also share the same radial velocity, retrograde orbit, and essentially the same photometric and trigonometric parallaxes. Studies by King (1997) and Fulbright (2000) find comparably low values of $\alpha$-element and neutron-capture element abundance ratios for this pair. Fulbright also finds $\mathrm{Na}$ underabundant $([\mathrm{Na} / \mathrm{Fe}]=$ $-0.58 \pm 0.04)$ and $\mathrm{Ni}$ very mildly so $([\mathrm{Ni} / \mathrm{Fe}]=-0.09 \pm$ 0.02). Employing King's (1997) abundance ratios of [( $\mathrm{Na}$, $\mathrm{Mg}, \mathrm{Si}) / \mathrm{Fe}]$, we derive $0.34 \pm 0.19<\left(N_{\mathrm{Ia}} / N_{\mathrm{II}}\right)_{\mathrm{CPM} \text { pair }}<$ $0.41 \pm 0.18$.
Of the three low- $\alpha$ stars analyzed for the present study, $\mathrm{BD}+80^{\circ} 245^{\prime}$ 's neutron-capture abundances are most similar to those of the low- $\alpha$ stars discussed in this section. It is plausible that $\mathrm{BD}+80^{\circ} 245$ is simply a lower metallicity representative of the more metal-rich low- $\alpha$ stars. A dynamical study, employing the orbital characteristics of the stars in this study, along with those of the more metal-rich low- $\alpha$ stars, might uncover a kinematic association between the objects. However, we find no halo stars in the literature that share the abundance trends of G4-36 and CS 22966-043.

\subsection{Kinematic and Chemical Connections to Dwarf Galaxy Environments?}

$\mathrm{BD}+80^{\circ} 245$ possesses a large apogalacticon distance of $\sim 20$ kpc (Carney et al. 1997; Fulbright 2002), which suggests that this object may not have been "born" within the halo but may have been accreted by the Galaxy along with other clusters and small galaxies (Carney et al. 1997). Both $\mathrm{BD}+80^{\circ} 245$ and G4-36 possess retrograde orbits (Carney et al. 1997; James 2000), a signature consistent with a capture scenario (Barnes 1996). No parallax information is available for CS 22966-043. However, with a radial velocity of roughly $-268 \mathrm{~km} \mathrm{~s}^{-1}$ and a position near the south Galactic pole, the star has a very high $W$ velocity. Combining proper-motion information from USNO B1.0 (Monet et

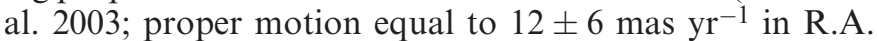
and $10 \pm 3$ mas $\mathrm{yr}^{-1}$ in declination) with an estimate of its photometric distance, CS 22966-043 clearly belongs to the outer halo population.

Recent high-resolution studies of the chemical compositions of stars in some of the nearby $\mathrm{dSph}$ galaxies show that the dwarf galaxies have undergone nucleosynthetic histories different both from those in our Galaxy and from each other. Of these dwarf galaxies, the abundance patterns of the Sagittarius dSph metal-poor stars most closely resemble some of the trends observed in the Galactic halo. For instance, the chemical abundances in Sgr dSph stars of $[\mathrm{Fe} / \mathrm{H}]<-1$ observed by Smecker-Hane \& McWilliam (2003) possess $[\alpha / \mathrm{Fe}]$ ratios comparable to those of most Milky Way halo stars. This $\alpha$-element abundance behavior is unlike that found by Shetrone et al. (2003) for their sample of low-metallicity stars in the Sculptor, Fornax, Carina, and Leo I dSph galaxies, where the abundances of $\alpha$ elements overall are lower than those found in Galactic halo stars of similar metallicities. Comparable underabundances of the $\alpha$-elements were found by Shetrone, Côte, \& Sargent (2001) for the Draco, Ursa Minor, and Sextans dSph galaxies.

Similar differences are observed in the neutron-capture element abundance ratios (e.g., $[\mathrm{Y} / \mathrm{Fe}]$ ) between those derived by Smecker-Hane \& McWilliam (2003) for stars in Sgr dSph galaxies and those derived by Shetrone et al. (2003) for stars in Sculptor, Fornax, Carina, and Leo I dSph galaxies. Furthermore, within the sample of galaxies observed by Shetrone et al. (2003), the behavior of the $[(\mathrm{Ba}, \mathrm{La}) / \mathrm{Eu}]$ abundance ratio pattern is different between Leo I and the others, providing further evidence of a variety of chemical enrichment histories.

Comparing the abundances derived in this study against those reported for individual dSph stars in the literature, we find that star Carina 5 not only exhibits $\alpha$-element abundances similar to those we find in $\mathrm{BD}+80^{\circ} 245$ but shares similarly low $\mathrm{Cu}$ and $\mathrm{Zn}$ abundances. However, Carina 5 does 
not resemble $\mathrm{BD}+80^{\circ} 245$ in the abundances of neutroncapture elements: the abundance ratios of $\mathrm{Y}, \mathrm{Ba}, \mathrm{La}$, and Eu reported by Shetrone et al. (2003) are all 0.8-2.0 dex higher than those we find in our low- $\alpha$ star. Recently, a Draco dSph star of $[\mathrm{Fe} / \mathrm{H}] \simeq-3$ has been found to possess $\mathrm{Sr}$ and $\mathrm{Ba}$ abundances that are from 0.6 to over $1.0 \mathrm{dex}$ lower than those found in $\mathrm{BD}+80^{\circ} 245$ (Fulbright 2003). However, the Draco star does not exhibit the same low- $\alpha$ abundance trend as $\mathrm{BD}+80^{\circ} 245$ or the other low- $\alpha$ stars in our study. Instead, the Draco star abundances are reminiscent of the two very low metallicity stars $([\mathrm{Fe} / \mathrm{H}] \simeq-3.4)$ found by McWilliam et al. (1995a) and Ryan et al. (1996) to exhibit mildly low $\alpha$-element abundances along with either low $\mathrm{Sr}$ or low $\mathrm{Sr}$ and $\mathrm{Ba}$ ratios relative to iron. In the present-day population of $\mathrm{dSph}$ galaxies orbiting the Galaxy, no abundance ratios have been reported that are similar to those found in G4-36 or CS 22966-043. However, with the wide variety of chemical enrichment histories presented by the present-day dSph galaxies, we cannot rule out the possibility that our stars may have originated in a system that is no longer separate from the Milky Way.

\section{SUMMARY AND CONCLUSIONS}

We present evidence of chemical substructure in the Galactic halo in the form of elemental abundances of three unusual stars, BD $+80^{\circ} 245$, G4-36, and CS 22966-043. These stars were all serendipitously discovered as having low $\alpha$-element and neutron-capture element abundances in the context of previous studies. In the present study we confirm the earlier reports and expand the number of chemical abundance analyses. All three of the stars display low $\alpha$-element abundances: the mean abundance ratio for $[(\mathrm{Mg}, \mathrm{Si}$, $\mathrm{Ca}) / \mathrm{Fe}$ in these stars is $0.68 \pm 0.07$ dex below the mean of halo stars of comparable metallicity. In addition, all three also display very depressed values of neutron-capture material: the mean abundance ratio for $[(\mathrm{Sr}, \mathrm{Ba}) / \mathrm{Fe}]$ in the low- $\alpha$ stars is $1.02 \pm 0.18$ dex below the halo mean. These results provide further evidence that chemical enrichment and star formation histories varied from region to region within the Milky Way halo.

Our low- $\alpha$ trio does not display uniform abundances of elements with atomic numbers $22 \leq A \leq 31$. $\mathrm{BD}+80^{\circ} 245$ exhibits diminished $\mathrm{Ti}(0.55 \pm 0.08$ dex below the halo mean) and diminished $\mathrm{Zn}$ but otherwise close to normal halo-type values of Fe peak abundances, whereas G4-36 and CS 22966-043 present mild to pronounced overabundances of these elements (from 0.2 to over 1 dex above the halo mean). In our analysis, G4-36 and CS 22966-043 require such large $\mathrm{Ga}$ enhancements to synthesize the observed spectra that it is possible, for the first time, to report $\mathrm{Ga}$ abundances in any metal-poor star.

Results for two other lower metallicity stars previously reported as possessing unusually low $\alpha$-element abundances, BD $+24^{\circ} 1676$ and G84-29, are less clear. Neither of these two stars displays diminished abundances of $\alpha$-elements in our study. Abundance enhancements we observe in $\mathrm{Mn}$ and $\mathrm{Ni}$, while comparable to those observed in G4-36 and CS 22966-043, are either based on only a single line or show a larger scatter than that observed for the same elements in the other program stars.

We explore the idea that the elemental abundance ratio diminishments in $\mathrm{BD}+80^{\circ} 245$, G4-36, and CS 22966-043 are the result of iron enhancements from SN Ia contribu- tions, by deriving the ratios of $\mathrm{SN}$ contributions ( $\mathrm{SNe} \mathrm{Ia} /$ SNe II). We sifted through combinations of SN Ia and SN II model yields to find the best matches to the chemical abundances we derived. Tests combining yields from various types of SN Ia scenarios and models with integrated SN II yields offer some insight but are of limited success in "explaining" the abundances of the low- $\alpha$ stars. There is simply insufficient reliable yield information available. In the best case, the solar case, only a handful of abundance ratios for relatively light elements (e.g., $\mathrm{Na}, \mathrm{Mg}, \mathrm{Si}$, and, possibly, O) can be modeled successfully using the available yields. For the Sun, applying our simple approach, we derive $0.18 \pm 0.01<\left(N_{\text {Ia }} / N_{\text {II }}\right)_{\odot}<0.25 \pm 0.06$, which is in good agreement with literature values of the Galactic ratios of SN Ia to SN II events.

The results for our program stars confirm the idea that the low- $\alpha$ abundances can be explained by a larger contribution of SN Ia yields than those that went into the average halo star. To summarize the findings presented in Table 12, $0.6 \lesssim\left(N_{\text {Ia }} / N_{\text {II }}\right)_{\text {New Pop }} \lesssim 1.3$. These ratios are in contrast to those found for other metal-poor field halo stars for which, as a result of the timescale of their formation, no significant contribution from $\mathrm{SNe}$ Ia is expected to be observed. Thus, BD $+80^{\circ} 245$, G4-36, and CS 22966-043 may have formed from material polluted by the earliest SN Ia events that occurred in the Milky Way. The unusual abundances of these stars in $\mathrm{Ti}, \mathrm{Cr}, \mathrm{Mn}, \mathrm{Ni}, \mathrm{Zn}$, and possibly $\mathrm{Cu}$ cannot be modeled, however, using existing SN Ia yields. An outstanding question of current interest is the determination of the SN Ia progenitors and the degree to which nonstandard progenitor scenarios contributed to the ISM. Trustworthy SN Ia and SN II yields for the Fe peak elements would allow us to determine the progenitor SNe Ia not only for our unusual program stars but for halo stars in general. To our knowledge, an identification or determination of SN Ia progenitors based on chemical abundances does not exist in the literature.

In the case of the three low- $\alpha$ stars we focus on in this study, these objects represent a nonnegligible part of early Galactic nucleosynthesis, yet understanding the origins of the abundances in these objects does not lie in current Galactic chemical evolution models. Clearly, the question of how these anomalous abundances arose requires a more detailed and rigorous confrontation with theoretical models.

This research has benefited from the use of NASA's Astrophysics Data System Bibliographic Services; the SIMBAD database, operated at CDS, Strasbourg, France; the NASA/IPAC Extragalactic Database, which is operated by the Jet Propulsion Laboratory, California Institute of Technology, under contract with NASA; and the Astronomical Data Center at NASA Goddard Space Flight Center. We warmly thank George Wallerstein, David Lambert, Judy Cohen, Bob Kraft, Dave Arnett, Stan Woosley, Alex Heger, Ken'ichi Nomoto, Wayne Baumgartner, John Norris, Roberto Gallino, Andy McWilliam, Mike Fall, Linda Rowan, Harriet Dinerstein, and Jennifer Simmerer for fruitful discussions and helpful information. We gratefully acknowledge Francesca Primas, Tamara Mishenina, Tammy Smecker-Hane, and Andy McWilliam for graciously conveying abundance results prior to publication. This research was supported via funding to I. I. I. from NASA through Hubble Fellowship grant 
HST-HF-01151.01-A from the Space Telescope Science Institute, operated by AURA, Inc., under NASA contract NAS 5-26555, and from the NSF through grants AST 9987162 to C. S., AST 99-88156 to B. W. C., and AST 0098644 to J. C. W. Furthermore, P. A. H. would also like to thank NASA for its support by NASA grant NAG 5-7937. Both I. I. I. and C. R. J. are also pleased to acknowledge graduate student support from the University of Texas at Austin through McDonald Observatory and the Department of Astronomy and by Graduate Fellowships to I. I. I. In addition, C. R. J. also acknowledges research support through the Research Enhancement Grant Program at Sam Houston State University.
Alecian, G., \& Stift, M. J. 2002, A\&A, 387, 27

Allende Prieto, C., Lambert, D., \& Asplund, M. 2001, ApJ, 556, L63

Alonso, A., Arribas, S., \& Martínez-Roger, C. 1996, A\&A, 313, 873 1999, A\&AS, 140, 261 2001, A\&A, 376, 1039

Anders, E., \& Grevesse, N. 1989, Geochim. Cosmochim. Acta, 53, 197

Arlandini, C., Käppeler, F., Wisshak, K., Gallino, R., Lugaro, M., Busso, M., \& Staniero, O. 1999, ApJ, 525, 886

Arnett, W. D. 1969, Ap\&SS, 5, 180

Arpigny, C., \& Magain, P. 1983, A\&A, 127, L7

Axer, M., Fuhrmann, K. \& Gehren, T. 1994, A\&A, 291,895

Barnes, J. E. 1996, in ASP Conf. Ser. 92, Formation of the Galactic HaloInside and Out, ed. H. L. Morrison \& A. Sarajedini (San Francisco: ASP), 415

Bartkevičius, A. 1996, Baltic Astron., 5, 217

Baumgartner, W. H., Mushotzky, R. F., \& Horner, D. J. 2003, in ASP Conf. Ser., Matter and Energy in Clusters of Galaxies, ed. S. Bowyer \& C.-Y. Hwang (San Francisco: ASP), in press

Baumüller, D. \& Gehren, T. 1997, A\&A, 325, 1088

Bergbusch, P. A., \& VandenBerg, D. A. 1992, ApJS, 81, 163

Biémont, E., Lyngå, C., Li, Z. S., Svanberg, S., Garnir, H. P., \& Doidge, P. S. 1999, MNRAS, 303, 721

Bond, H. E. 1974, ApJ, 194, 95

Brown, J. A., Wallerstein, G., \& Zucker, D. 1997, AJ, 114, 180

Buonanno, R., Buscema, G., Fusi Pecci, F., Richer, H. B., \& Fahlman, G. G. 1990, AJ, 100, 1811

Burris, D. L., Pilachowski, C. A., Armandroff, T. A., Sneden, C., Cowan, J. J., \& Roe, H. 2000, ApJ, 544, 302

Cameron, A. G. W. 2001, ApJ, 562, 456

Carney, B. W., \& Latham, D. W. 1985, ApJ, 298, 803

Carney, B. W., Latham, D. W., Laird, J. B., \& Aguilar, L. A. 1994, AJ, 107, 2240

Carney, B. W., Latham, D. W., Laird, J. B., Grant, C. E., \& Morse, J. A. 2001, AJ, 122, 3419

Carney, B. W., Wright, J. S., Sneden, C., Laird, J. B., Aguilar, L. A., \& Latham, D. W. 1997, AJ, 114, 363

Carretta, E., Gratton, R., Cohen, J. G., Beers, T. C., \& Christlieb, N. 2002, AJ, 124, 481

Caretta, E., Gratton, R. G., \& Sneden, C. 2000, A\&A, 356, 238

Castelli, F., Gratton, R. G., \& Kurucz, R. L. 1997, A\&A, 318, 841

Cohen, J. G., Christlieb, N., Beers, T. C., Gratton, R., \& Carretta, E. 2002, AJ, 124,470

Cowan, J. J., Burris, D. L., Sneden, C., McWilliam, A., \& Preston, G. W. 1995, ApJ, 439, L51

Cowan, J. J., McWilliam, A., Sneden, C., \& Burris, D. L. 1997, ApJ, 480, 246

Cowan, J. J., et al. 2002, ApJ, 572, 861

Domínguez, I., Höflich, P., \& Straniero, O. 2001, ApJ, 557, 279

ESA 1997, The Hipparcos and Tycho Catalogues (ESA SP-1200; Noordjwik: ESA)

Fitzpatrick, M. J., \& Sneden, C. 1987, BAAS, 19, 1129

Francois, P., Spite, M., \& Spite, F. 1993, A\&A, 274, 82

Freiburghaus, C., Rosswog, S., \& Thielemann, F.-K. 1999, ApJ, 525, L121

Fuhrmann, K. 1998, A\&A, 330, 626

Fuhrmann, K., Axer, M., \& Gehren T. 1995, A\&A, 301, 492

Fujimoto, M. Y., Nomoto, K., \& Sugimoto, D. 1976, Ap\&SS, 45, 71

Fulbright, J. P. 1999, Ph.D. thesis, Univ. California, Santa Cruz $2000, \mathrm{AJ}, 120,184$

2002, AJ, 123, 404 2003, ApJ submitte

Fulbright, J. P., \& Kraft, R. P. 1999, AJ, 118, 527

Gilroy, K. K., Sneden, C., Pilachowski, C. A., \& Cowan, J. J. 1988, ApJ, 327,298

Giridhar, S., Lambert, D. L., Gonzalez, G., \& Pandey, G. 2001, PASP, 113, 519

Gratton, R. G. 1989, A\&A, 208, 171

Gratton, R. G., \& Ortolani, S. 1988, A\&AS, 73, 137

Gratton, R. G., \& Sneden, C. 1991, A\&A, 241, 501 1994, A\&A, 287, 927

Grevesse, N., \& Sauval, A. J. 1998, Space Sci. Rev., 85, 161

Harris, W. E. 1996, AJ, 112, 1487

Hashimoto, M., Nomoto, K. \& Thielemann, F.-K. 1989, A\&A. 210, L5

Hashimoto, M., Nomoto, K., Tsujimoto, T., \& Thielemann, F.-K. 1996, in IAU Colloq. 145, Supernovae and Supernova Remnants, ed. R. McCray \& Z. Wang (Cambridge: Cambridge Univ. Press), 157

\section{REFERENCES}

Hix, W. R., \& Thielemann, F.-K. 1996, ApJ, 460, 869

Hobbs, L. M., \& Pilachowski, C. 1988, ApJ, 326, L23

Höflich, P., Gerardy, C. L., Fesen, R. A., \& Sakai, S. 2002, ApJ, 568, 791

Höflich, P., \& Khokhlov, A. 1996, ApJ, 457, 500

Höflich, P., Khokhlov, A. M., \& Wheeler, J. C. 1995, ApJ, 444, 831

Höflich, P., Wheeler, J. C., \& Thielemann, F. K. 1998, ApJ, 495, 617

Holweger, H. 2001, in AIP Conf. Proc. 598, Solar and Galactic Composition: A Joint $\mathrm{SOHO} / \mathrm{ACE}$ Workshop, ed. R. F. Wimmer-Schweingruber (New York: AIP), 23

Hoyle, F., \& Fowler, W. A. 1960, ApJ, 132, 565

Iwamoto, K., Brachwitz, F., Nomoto, K., Kishimoto, N., Umeda, H., Hix, W. R., \& Thielemann, F.-K. 1999, ApJS, 125, 439

James, C. R. 1998, BAAS, 20, 1321

2000, Ph.D. thesis, Univ. Texas, Austin

Johnson, J. A. 2002, ApJS, 139, 219

King, J. R. 1997, AJ, 113, 2302

Kinman, T. D. 1999 , Ap\&SS, 267, 83

Korn, A. J., Shi, J.-R., \& Gehren, T. 2003, A\&A, submitted

Kraft, R. P., \& Ivans, I. I. 2003, PASP, 115, 143

Kurucz, R. L. 1993, Kurucz CD-ROM 13, ATLAS 9 Stellar Atmosphere Programs and $2 \mathrm{~km} / \mathrm{s}$ Grid (Cambridge: SAO)

Kurucz, R. L., \& Bell, B. 1995, Kurucz CD-ROM 23, Atomic Line List (Cambridge: SAO)

Lambert, D. L., Heath, J. E., Lemke, M., \& Drake, J. 1996, ApJS, 103, 183

Latham, D. W., Stefanik, R. P., Torres, G., \& Davis, R. J. 2002, AJ, 124, 1144

Magain, P. 1989, A\&A, 209, 211

McClure, R. D. 1997, PASP, 109, 536

McClure, R. D., \& Woodsworth, A. W. 1990, ApJ, 352, 709

McWilliam, A. 1997, ARA\&A, 35, 503

. 1998, AJ, 115, 1640

McWilliam, A., Preston, G. W., Sneden, C., \& Searle, L. 1995a, AJ, 109, 2757

McWilliam, A., Preston, G. W., Sneden, C., \& Shectman, S. 1995b, AJ, 109,2736

McWilliam, A., \& Searle, S. 1999, Ap\&SS, 265, 133

Michaud, G. 1970, ApJ, 160, 641

Mishenina, T. V., \& Kovtyukh, V. V. 2001, A\&A, 370, 951

Mishenina, T. V., Kovtyukh, V. V., Soubiran, C., Travaglio, C., \& Busso, M. 2002, A\&A, 396, 189

Monet, D. G., et al. 2003, AJ, 125, 984

Moore, C. E., Minnaert, M. G. J., \& Houtgast, J. 1966, The Solar Spectrum 2935 A to $8770 \AA$ A (NBS Monogr. 61; Washington, DC: US Gov. Printing Office)

Nielsen, K., Karlsson, H., \& Wahlgren, G. M. 2000, A\&A, 363, 815

Nissen, P. E., Gustafsson, B., Edvardsson, B., \& Gilmore, G. 1994, A\&A, 285,440

Nissen, P. E., Primas, F., Asplund, M., \& Lambert, D. L. 2002, A\&A, 390, 235

Nissen P. E., \& Schuster W. J. 1997, A\&A, 326, 751

Nomoto, K., \& Hashimoto, M. 1988, Phys. Rep., 163, 13

Nomoto, K., Sugimoto, D., \& Neo, S. 1976, Ap\&SS, 39, L37

Nomoto, K., Thielemann, F.-K., \& Yokoi, K. 1984, ApJ, 286, 644

Pagel, B. E. G., \& Tautvaisiene, G. 1995, MNRAS, 276, 505

Peterson, R. C., Dorman, B., \& Rood, R. 2001, ApJ, 559, 372

Preston, G. W. 1974, ARA\&A, 12, 257

Preston, G. W., \& Landolt, A. U. 1998, AJ, 115, 2515 1999, AJ, 118, 3006

Preston, G. W., \& Sneden, C. 2000, AJ, 120, 1014

Primas, F., Brugamyer, E., Sneden, C., King, J. R., Beers, T. C., Boesgaard, A. M., \& Deliyannis, C. P. 2000, in The First Stars, ed. A. Weiss, T. G. Abel, \& V. Hill (New York: Springer), 51

Prochaska, J. X., \& McWilliam, A. 2000, ApJ, 537, L57

Raiteri, C. M., Gallino, R., \& Busso, M. 1992, ApJ, 387, 263

Reetz, J. 1999, Ap\&SS, 265, 171

Richer, J., Michaud, G., \& Turcotte, S. 2000, ApJ, 529, 338

Rosswog, S., Liebendörfer, M., Thielemann, F.-K., Davies, M. B., Benz, W., \& Piran, T. 1999, A\&A, 341, 499

Ryan, S. G., Norris, J. E., \& Beers, T. C. 1996, ApJ, 471, 254

Sadakane, K., Jugaku, J., \& Takada-Hidai, M. 1988, ApJ, 325, 776

Salpeter, E. E. 1955, ApJ, 121, 161

Schectman, S. A. 1984, Proc. SPIE, 445, 128

Schlegel, D. J., Finkbeiner, D. P., \& Davis, M. 1998, ApJ, 500, 525

Schuster, W. J., \& Nissen, P. E. 1989, A\&A, 222, 69

Shetrone, M. D., Côte, P., \& Sargent, W. L. W. 2001, ApJ, 548, 592 
Shetrone, M. D., Venn, K. A., Tolstoy, E., Primas, F., Hill, V., \& Kaufer, A. 2003, AJ, 125,684

Smecker-Hane, T. A., \& McWilliam, A. 2003, ApJ, submitted

Sneden, C. 1973, ApJ, 184, 839

Sneden, C., Cowan, J. J., Ivans, I. I., Fuller, G. M., Burles, S., Beers, T. C., \& Lawler, J. E. 2000, ApJ, 533, L139

Sneden, C., Cowan, J. J., Lawler, J. E., Burles, S., Beers, T. C., \& Fuller, G. M. 2002, ApJ, 566, L25

Sneden, C., \& Crocker, D. A. 1988, ApJ, 335, 406

Sneden, C., Gratton, R. G., \& Crocker, D. A. 1991a, A\&A, 246, 354

Sneden, C., Kraft, R. P., Prosser, C. F., \& Langer, G. E. 1991b, AJ, 102, 2001

Sneden, C., McWilliam, A., Preston, G. W., Cowan, J. J., Burris, D. L., \& Armosky, B. J. 1996, ApJ, 467, 819

Sneden, C., Preston, G. W., McWilliam, A., \& Searle, L. 1994, ApJ, 431, L27

Sneden, C., et al. 2003, ApJ, in press

Spite, M., \& Spite, F. 1978, A\&A, 67, 23

Stephens, A. \& Boesgaard, A. M. 2002, AJ, 123, 1647

Straniero, O., Chieffi, A., \& Limongi, M. 1997, ApJ, 490, 425

Takada-Hidai, M., Sadakane, K., \& Jugaku, J. 1986, ApJ, 304, 425

Terasawa, M., Sumiyoshi, K., Yamada, S., Suzuki, H., \& Kajino, T. 2002, ApJ, 578, L137

Thévenin, F. 1998, Chemical Abundances in Late-Type Stars

Thévenin, F., \& Idiart, T. P. 1999, ApJ, 521, 753

Thielemann, F.-K., Nomoto, K., \& Hashimoto, M.-A. 1996, ApJ, 460, 408
Thielemann, F.-K., Nomoto K., \& Yokoi, K. 1986, A\&A, 158, 17

Thompson, T. A., Burrows, A., \& Meyer, B. S. 2001, ApJ, 562, 887

Timmes, F. X., Woosley, S. E., \& Weaver, T. A. 1995, ApJS, 98, 617

Tinsley, B. M. 1979, ApJ, 229, 1046

Tsujimoto, T., Nomoto, K., Yoshii, Y., Hashimoto, M., Yanagida, S., \& Thielemann, F.-K. 1995, MNRAS, 277, 945

Tsujimoto, T., Shigeyama, T., \& Yoshii, Y. 2002, ApJ, 565, 1011

Tull, R. G., MacQueen, P. J., Sneden, C., \& Lambert, D. L. 1995, PASP, 107,251

Umeda, H., \& Nomoto, K. 2002, ApJ, 565, 385

van den Bergh, S., \& Tammann, G. A. 1991, ARA\&A, 29, 363

van Dyk, S. D. 1992, AJ, 103, 1788

Vogt, S. S., et al. 1994, Proc. SPIE, 2198, 362

Wallerstein, G. 1962, AJ, 67, 122

Wanajo, S., Itoh, N., Ishimaru, Y., Nozawa, S., \& Beers, T. C. 2002, ApJ, 577,853

Wanajo, S., Tamamura, M., Itoh, N., Nomoto, K., Ishimaru, Y., Beers, T. C., \& Nozawa, S. 2003, ApJ, in press

Weiler, K. W., \& Sramek, R. A. 1988, ARA\&A, 26, 295

Wheeler, J. C. 2003, Am. J. Phys., 71, 11

Woosley, S. E., Langer, N., \& Weaver, T. A. 1995, ApJ, 448, 315

Woosley, S. E., \& Weaver, T. A. 1995, ApJS, 101, 18

Yi, S., Demarque, P., Kim, Y.-C., Lee, Y.-W., Ree, C. H., Lejeune, T., \& Barnes, S. 2001, ApJS, 136, 417

Zhao, G., \& Magain, P. 1990, A\&A, 238, 242 